\title{
Astrophysical Smooth Particle Hydrodynamics
}

\author{
Stephan Rosswog \\ School of Engineering and Science, Jacobs University Bremen, Campus Ring 1, \\ 28759 Bremen, Germany
}

\begin{abstract}
The paper presents a detailed review of the smooth particle hydrodynamics ( $\mathrm{SPH}$ ) method with particular focus on its astrophysical applications. We start by introducing the basic ideas and concepts and thereby outline all ingredients that are necessary for a practical implementation of the method in a working SPH code. Much of SPH's success relies on its excellent conservation properties and therefore the numerical conservation of physical invariants receives much attention throughout this review. The self-consistent derivation of the SPH equations from the Lagrangian of an ideal fluid is the common theme of the remainder of the text. We derive a modern, Newtonian SPH formulation from the Lagrangian of an ideal fluid. It accounts for changes of the local resolution lengths which result in corrective, so-called "grad-h-terms". We extend this strategy to special relativity for which we derive the corresponding grad-h equation set. The variational approach is further applied to a general-relativistic fluid evolving in a fixed, curved background space-time. Particular care is taken to explicitely derive all relevant equations in a coherent way.
\end{abstract}

Key words: Hydrodynamics, variational principles, conservation, shocks, relativity $P A C S$ : 


\section{Contents}

1 Introduction 3

2 Basic concepts of Smooth Particle Hydrodynamics 8

$\begin{array}{lr}\text { 2.1 Lagrangian hydrodynamics } & 8\end{array}$

2.2 The SPH kernel interpolation $\quad 9$

2.3 The "vanilla ice" SPH equations 13

$\begin{array}{ll}2.4 \text { Conservation properties } & 16\end{array}$

$\begin{array}{ll}2.5 \text { Alternative SPH discretizations } & 17\end{array}$

$\begin{array}{ll}2.6 \text { Adaptive resolution } & 19\end{array}$

$\begin{array}{ll}2.7 \text { Artificial dissipation } & 20\end{array}$

2.8 Time integration in SPH 29

2.9 "Best practice" suggestions 36

$\begin{array}{lll}3 & \text { SPH from a variational principle } & 38\end{array}$

3.1 The Lagrangian and the Euler-Lagrange equations 38

3.2 The density, its derivatives and "grad-h"-terms 39

3.3 The SPH equations with "grad-h"-terms 40

4 Relativistic SPH 42

4.1 Special-relativistic SPH 43

4.2 General-relativistic SPH on a fixed background metric $\quad 54$

$\begin{array}{lll}5 & \text { Summary } & 64\end{array}$

$\begin{array}{ll}\text { References } & 66\end{array}$ 


\section{Introduction}

Much of what we observe in the physical Universe has been shaped by fluid dynamical processes. From the hot gas in galaxy clusters to the internal structures of their consituent galaxies down to their stars and planets, all has been formed by the interplay between gravity, gas dynamics and further physical processes such as the interaction with radiation, nuclear burning or magnetic fields. The latter processes often involve intrinsic length and time scales that are dramatically different from those of the gas dynamical processes, therefore many astrophysical problems are prime examples of multi-scale and multiphysics challenges. The complexity of the involved physical processes and the lack of symmetry usually prohibit analytical treatments and only numerical approaches are feasible.

Although fluid dynamics is also crucial for many technical applications, their requirements usually differ substantially from those of astrophysics and this also enters the design of the numerical methods. Typical astrophysical requirements include:

- Since fixed boundaries are usually absent, flow geometries are determined by the interplay between different physical processes such as gas dynamics and (self-)gravity which often lead to complicated, dynamically changing flow geometries. Thus, a high spatial adaptivity is often required from astrophysical hydodynamics schemes.

- Shocks often crucially determine the evolution of cosmic objects. Examples include in supernova remnants or the Earth's magnetosphere.

- Physical quantities can vary by many orders of magnitudes between different regions of the simulation domain. This requires a particularly high robustness of the numerical scheme.

- In many astrophysical problems the numerical conservation of physically conserved quantities determines the success and the reliability of a computer simulation. Consider, for example, a molecular gas cloud that collapses under the influence of its own gravity to form stars. If the simulation for some reason dissipates angular momentum, a collapsing, self-gravitating portion of gas may form just a single stellar object instead of a multiple system of stars and it will thus produce a qualitatively wrong result.

- Many astrophysical questions require dealing with physical processes beyond gas dynamics and self-gravity. A physically intuitive and flexible formulation of the numerics can substantially facilitate the implementation of new physics modules into existing codes.

No numerical method performs equally well at each of the above requirements, therefore, the choice of the best-suited numerical approach can often save a tremendous amount of effort in obtaining reliable results. Therefore: horses for courses. 
In the following, the smooth particle hydrodynamics (SPH) method [1-6], a completely mesh-free approach to solve the hydrodynamic equations is discussed in detail. Its conservation properties are a major strength of SPH, therefore "hard-wired" conservation receives much attention throughout this text. The derivation of the SPH equations (in the absence of dissipation) requires nothing more than a suitable Lagrangian, a density prescription that depends on the coordinates and the first law of thermodynamics. The resulting equations conserve the physically conserved quantities even in their discretized form, provided that the original Lagrangian possessed the correct symmetries. Therefore, derivations from Lagrangians play a central role in our discussion of the subject.

This review has emerged from a lecture series on "Computational relativistic astrophysics" as part of a Doctoral Training Programme on the "Physics of Compact Stars" that was held in summer 2007 at the European Centre for Theoretical Studies in Nuclear Physics and Related Areas (ECT*) in Trento, Italy. In the pedagogical spirit of this lecture series the text is kept in "lecture" rather than "paper" style, i.e. even rather trivial steps are written down explicitely. The goal is to pave a broad and smooth avenue to a deep understanding of the smooth particle hydrodynamics method rather than just to provide a bumpy trail. Due to this pedagogical scope, the focus of this review needs to be clear-cut, but rather narrow: it only discusses the numerical solution of the inviscid hydrodynamics equations, in the Newtonian, specialrelativistic and general-relativistic (fixed metric) case.

For practical astrophysical simulations often sophisticated additional physics modules are required and their implementation may pose additional numerical challenges which are beyond the scope of this review. In the following we provide a brief list of additional physics that has been implemented into SPH and point to references that are intended as starting points for further reading.

\section{- Gravity:}

Self-gravity is for many astrophysical problems a key ingredient. A straight forward calculation of pairwise gravitational forces between $N$ particles requires a prohibitively large $O\left(N^{2}\right)$ number of operations and therefore such an approach is not feasible for realistic problems. The most natural gravitational force evaluation for a purely meshfree method like SPH is the use of a tree, either in the form of an oct-tree [7,3,8-12] or a binary tree [1315] both of which require only $O(N \log (N))$ operations. Other possibilities include particle-mesh methods [16-18] in which the particles are mapped onto a mesh. The latter is used to efficiently solve Poisson's equation and to subsequently calculate the accelerations at the particle positions. Dehnen $[19,20]$ has used ideas from the Fast Multipole Method (FMM) [21] for a fast tree code which scales proportional to $O(N)$. Several hybrid approaches which combine elements of different methods exist [22-26]. 
- Equations of state:

For some problems polytropic equations of state (EOS), $p=K \rho^{\Gamma}$, with $K$ being a function of entropy, $\rho$ the matter density and $\Gamma$ the adiabatic exponent, are sufficiently accurate. Other problems require more sophisticated approaches. This is particularly true in cases where the matter compressibility depends on the density regime, as, for example, in neutron stars where the effective polytropic exponent above nuclear matter density is $\Gamma \approx 2.5$, but drops to values close to $4 / 3$ at lower densities. Different nuclear EOSs have been used [27-31] in the context of neutron stars, at lower densities and in cases where the matter composition is important for other processes further physical EOSs have been applied [32-36].

\section{- Solid state mechanics}

In the context of planetary impacts additional concepts such as material stresses, fracture physics or plasticity criteria need to be implemented into $\mathrm{SPH}$. This branch of development was initiated by the work of [37] and further continued by [38-45]. More recently, also porosity models were included in SPH $[46,47]$.

\section{- Physical viscosity:}

For some applications the solution of the non-dissipative hydrodynamics equations is inadequate and physical (as opposed to artificial) viscosity needs to be modelled. Examples include the angular momentum transport in accretion disks, see e.g. [48], or the dissipation of sound waves as a mechanism to heat intra-cluster media [49]. Various authors have implemented the viscous stress tensor into SPH [50-56]. This requires particular care in the implementation of higher order derivatives which -in a straight forward kernel approximation- can be sensitive to particle disorder.

\section{- Thermal conduction:}

Thermal conduction has, for example, been proposed as a possible heating mechanism for offsetting central cooling losses in rich clusters of galaxies. Its implementation poses some challenges due to second derivatives that enter the conductive term of the energy equation. Usually, a particluar discretization of the second derivative due to [57] is used in modelling thermal conduction $[58,59]$.

\section{- Nuclear burning:}

Often the energy release due to nuclear reactions is too slow to influence gas flows substantially on a dynamical time scale. Under special circumstances, however, explosive nuclear burning can be triggered and in such cases the nuclear energy release can become the main driver of the dynamical gas evolution. In such cases the coupling between the hydrodynamics and the nuclear reactions is challenging due the huge difference in their intrinsic time scales. The first implementation of a nuclear reaction network into 
SPH goes back to [60]. The 14 isotope $\alpha$-chain network of this first study has also been used with updated nuclear reaction rates in studies of white dwarf coalescences [32]. More recently, further small networks [61,62] have been coupled with SPH [36] to study white dwarf coalescences [35,63], collisions between them $[64,65]$ and tidal disruptions of white dwarfs by black holes $[66,67]$.

- Chemistry:

Chemical abundances carry the imprints of the past evolutionary processes that shaped today's galaxies. Their chemical enrichment is inseparably interweaved with the formation of structure in galaxies since -being closely related to supernovae- it drives local energy feedback and gas expansion, but on the other hand it also triggers - via metallicity-dependent cooling- the contraction and collapse of local gas structures. A large variety of chemical evolution approaches has been implemented into $\mathrm{SPH}$, for a starting point one may want to consult [68-79].

- Radiation: photons and neutrinos

Closely related to chemical species is the interaction between photons and the ambient gas. For some problems relatively simple cooling and heating recipes can be applied, e.g. [3,80,12]. Optically thick diffusion has in fact already been implemented in one of the very first SPH-papers [1]. Later, more sophisticated approaches to calculate the required second derivatives were suggested [57]. For a recent implicit diffusion approach, see [81]. Fluxlimited diffusion schemes have been suggested by [82-84], Monte-Carlo-type approaches have been followed by [85-88] and ray-tracing has been applied by [89-91]. Several further methods to treat radiation in SPH do exist [9297].

When matter densities are so large that photons are "trapped", neutrinos can become the only cooling agents. For white dwarfs and less compact stars matter is transparent to neutrinos and they can just be treated as a local drain of thermal energy. For higher densities, say in a neutron star or the inner regions of the disk around a hyper-accreting black hole, opacity effects may prohibit the free escape of neutrinos. A further example of the astrophysical importance of neutrino physics is the delayed explosion mechanism $[98,99]$ of core-collapse supernovae where the energy deposition via neutrinos is thought to be vital to re-energize the stalled shock after it has crossed the outer layers of the stellar iron core. In this context, multi-flavour neutrino cooling and heating has been implemented into $\mathrm{SPH}[27,31]$ in an entirely particle-based, flux-limited diffusion approach. To model compact object mergers, neutrinos have been implemented into SPH via a hybrid, particle-mesh approach [100] and by using local disk scale heights to estimate neutrino opacities [33,34]. 
- Magnetic fields:

Magnetic fields are prevalent everywere in the cosmos, but they pose a notorious numerical challenge not least because of the difficulty in fulfilling the $\nabla \cdot \vec{B}=0$-constraint. The most obvious approach is a straight-forward SPH discretization of the ideal MHD equations [2,101-106]. While it performs reasonably well in some problems, particle disorder and finite $\nabla \cdot \vec{B}$ values can trigger numerical instabilities [105]. Several approaches have been developed that apply regularization techiques to overcome the problems related to particle disorder [107-111]. Recently, an approach has been implemented $[112,113]$ that advects so-called Euler-potentials, $\alpha$ and $\beta$, with the SPH particles. The magnetic field can be reconstructed from the values of these potentials at the particle positions via $\vec{B}=\nabla \alpha \times \nabla \beta$. This approach guarantees the fulfillment of the $\nabla \cdot \vec{B}=0$-constraint by construction and has shown excellent results in a large number of test cases [112].

- Further "subgrid" models:

Often, numerical models of astrophysical processes require the feedback from physical processes on sub-resolution scales. Such processes are usually implemented as (parametrized) "subgrid" models that transmit the main effects from the unresolved processes to the fluid. Cosmic rays present one such example. They contribute significantly to the pressure of the interstellar medium in our Galaxy and therefore they may be important in regulating star formation during the formation and evolution of galaxies. For the implementation of the effects of cosmic rays into SPH see [114-118]. Another example is the modelling of the feedback of (unresolvable) accretion-driven outflows during the merger of galaxies to study the self-regulation of black hole growth $[119,120]$. In simulations on galactic scales stars -their birth, feedback via outflows and possible supernova explosions- also need to be modelled as effective sub-scale models. By their very nature such approaches are closely linked to those of radiative transfer and chemical enrichment. For the various approaches we refer to the literature [121-143,12,144,145].

The remainder of this review is organized as follows. In Sec. 2 the most basic form of SPH ("vanilla ice") is derived by discretizing the equations of Lagrangian hydrodynamics. This form is somewhat ad hoc, yet it performs well in practice and it is at the heart of many SPH codes that are regularly used in the astrophysics community. In Sec. 3, the Lagrangian of an ideal fluid is discretized and subsequently used to derive a modern version of SPH. Since it follows from a variational principle, this formulation avoids any ambiguity with respect to a symmetrization of the equations and it naturally leads to additional terms that result from derivatives of the local hydrodynamic resolution length, the so-called "grad-h-terms", [146,147]. Armed with this gadgetery suitable Lagrangians are used to elegantly derive the SPH-equations both for the special- and the general-relativistic case, see Sec. 4. The summary and a brief outlook are presented in Sec. 5 . 


\section{Basic concepts of Smooth Particle Hydrodynamics}

\subsection{Lagrangian hydrodynamics}

In contrast to grid-based (Eulerian) methods, Smooth Particle Hydrodynamics (SPH) is purely Lagrangian. In the Eulerian picture, derivatives are calculated at a fixed point in space while, in the Lagrangian description, they are evaluated in a coordinate system attached to a moving fluid element. Thus in the Lagrangian approach we follow individual fish rather that staring at the pond and watch the swarm pass by. The Lagrangian (or substantial) time derivative, $d / d t$, is related to the Eulerian time derivative, $\partial / \partial t$, by

$$
\frac{d}{d t}=\frac{d x^{i}}{d t} \frac{\partial}{\partial x^{i}}+\frac{\partial}{\partial t}=\vec{v} \cdot \nabla+\frac{\partial}{\partial t}
$$

Applied to the Eulerian continuity equation,

$$
\frac{\partial \rho}{\partial t}+\nabla \cdot(\rho \vec{v})=0
$$

one finds, using $d \rho / d t=\vec{v} \cdot \nabla \rho+\partial \rho / \partial t$, the Lagrangian form

$$
\frac{d \rho}{d t}=-\rho \nabla \cdot \vec{v}
$$

The momentum conservation equation for a non-viscous fluid, the so-called Euler equation, reads in Lagrangian form

$$
\frac{d \vec{v}}{d t}=-\frac{\nabla P}{\rho}+\vec{f}
$$

i.e. apart from "body forces" such as gravity or magnetic fields embodied in the quantity $\vec{f}$, the fluid is accelerated by gradients of the pressure $P$. The energy equation follows directly from the (adiabatic) first law of thermodynamics, Eq. (7), together with Eq. (3):

$$
\frac{d u}{d t}=\frac{P}{\rho^{2}} \frac{d \rho}{d t}=-\frac{P}{\rho} \nabla \cdot \vec{v} .
$$




\section{First law of thermodynamics}

For SPH one needs the first law of thermodynamics, $d U=d Q-P d V$, in terms of "specific" quantities. Restriction to adiabatic processes allows to drop the $d Q$-term. On a "per mass basis", the energy $U$ becomes $u$ ("energy per mass") and the volume, $V$, becomes "volume per mass", i.e. $1 / \rho$, with $d V \rightarrow d(1 / \rho)=$ $-d \rho / \rho^{2}$. For the case without entropy generation the first law reads

$$
d u=\frac{P}{\rho^{2}} d \rho
$$

from which

$$
\frac{d u}{d t}=\frac{P}{\rho^{2}} \frac{d \rho}{d t} \quad \text { and } \quad\left(\frac{\partial u}{\partial \rho}\right)_{s}=\frac{P}{\rho^{2}}
$$

follow. In a relativistic context it can be advantageous to work with quantities "per baryon":

$$
\left(\frac{\partial u}{\partial n}\right)_{s}=\frac{P}{n^{2}}
$$

where $n$ is the baryon number density in the local rest frame.

The set of equations (3), (4), (5) must be closed by an equation of state that relates quantities such as the pressure, $P$, or the speed of sound, $c_{s}$, to macroscopic fluid quantities such as density or temperature. All the microphysics, say whether the pressure is produced by collisions in a Maxwell-Boltzmann gas or by degenerate electrons due to the Pauli principle, is embodied in the equation of state. It can be as simple as a polytrope, or, for more complicated cases, say for hot nuclear matter, it may only be available in tabular form, see the introduction for a link to the existing literature.

\subsection{The SPH kernel interpolation}

In the following, discrete representations of the continuous Lagrangian hydrodynamics equations are derived. In SPH, the interpolation points ("particles") are moved with the local fluid velocity ${ }^{1}$, derivatives are calculated via a kernel approximation without the need for finite differences. In this way the partial differential equations of Lagrangian fluid dynamics are transformed into ordi-

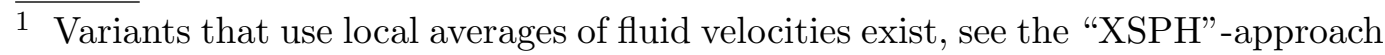
of [148]. 
nary differential equations.

In principle, there is some freedom in discretizing the fluid equations. But to ensure that the physically conserved quantities are also conserved by construction in the discretized particle equations the latter need to possess the correct symmetries in the particle indices. In the simplest, "vanilla ice" version of SPH, the symmetrization is imposed somewhat ad hoc, yet, it works well in practice and it is commonly used throughout astrophysics, see e.g. $[4,149,150,135,14,30,151]$. For different symmetrization possibilities see $[3,152,50,153]$ and Sec. 2.5.

\subsubsection{Interpolating function values}

At the heart of SPH is a kernel approximation in which a function $f(\vec{r})$ is approximated by

$$
\tilde{f}_{h}(\vec{r})=\int f\left(\overrightarrow{r^{\prime}}\right) W\left(\vec{r}-\overrightarrow{r^{\prime}}, h\right) d^{3} r^{\prime}
$$

where $W$ is the so-called smoothing kernel (or window function) and the smoothing length, $h$, determines the width of this kernel. Obviously, one would like to recover the original function in the limit of an infinitely small smoothing region and therefore the kernel should fulfill

$$
\lim _{h \rightarrow 0} \tilde{f}_{h}(\vec{r})=f(\vec{r}) \text { and } \int W\left(\vec{r}-\overrightarrow{r^{\prime}}, h\right) d^{3} r^{\prime}=1
$$

i.e. apart from being normalized, the kernel should have the $\delta$-distribution property in the limit of vanishing smoothing length.

To arrive at a discrete approximation, one can write the integral as

$$
\tilde{f}_{h}(\vec{r})=\int \frac{f\left(\overrightarrow{r^{\prime}}\right)}{\rho\left(\overrightarrow{r^{\prime}}\right)} W\left(\vec{r}-\overrightarrow{r^{\prime}}, h\right) \rho\left(\overrightarrow{r^{\prime}}\right) d^{3} r^{\prime}
$$

where $\rho$ is the mass density and subsequently one can replace the integral by a sum over a set of interpolation points ("particles"), whose masses, $m_{b}$, result from the $\rho\left(\vec{r}^{\prime}\right) d^{3} r^{\prime}$ term $^{2}$

$$
f(\vec{r})=\sum_{b} \frac{m_{b}}{\rho_{b}} f_{b} W\left(\vec{r}-\vec{r}_{b}, h\right)
$$

2 For simplicity, we are omitting the subscript $h$ in what follows. We also drop the distinction between the function to be interpolated and the interpolant, i.e. we use the same symbol $f$ on both sides of the equal signs. Throughout the article subscripts such as $A_{b}$ refer to the values at a particle position, i.e. $A_{b} \equiv A\left(\vec{r}_{b}\right)$. 
The summation interpolant Eq. (12) of the density then reads

$$
\rho(\vec{r})=\sum_{b} m_{b} W\left(\vec{r}-\vec{r}_{b}, h\right) .
$$

This density estimate by summing up kernel-weighted masses in the neighborhood of the point $\vec{r}$ plays a central role in the derivation of the SPH equations from a Lagrangian, see Sec. 3. Note that $h$ has not been specified yet, this will be addressed at a later point. In practice, the density can also be calculated via integration of a discretized version of Eq. (3) via Eq. (31), see below. In most applications the difference between integrated and summed density estimates is completely negligible. The summation form, however, is certainly numerically more robust, integration can, in extremely underresolved regions, produce negative density values. The summation form does not assume the density to be a differentiable function while Eqs. (3)/(31) do so [154]. The standard practice in SPH is to keep the particle masses fixed so that the mass conservation is perfect and there is no need to solve the continuity equation.

\subsubsection{Approximating derivatives}

To calculate derivatives, one takes the analytical expression of the summation approximation, Eq. (12),

$$
\nabla f(\vec{r})=\sum_{b} \frac{m_{b}}{\rho_{b}} f_{b} \nabla W\left(\vec{r}-\vec{r}_{b}, h\right),
$$

i.e. the exact derivative of the approximated function is used. The kernel function is known analytically, therefore there is no need for finite difference approximations.

Processes such as diffusion or thermal conduction require second derivatives. One could now proceed in a straight forward manner and take another derivative of Eq. (14). Yet, this estimate is rather sensitive to particle disorder and therefore not recommended for practical use. A better prescription is [57]

$$
\left(\nabla^{2} f\right)_{a}=2 \sum_{b} \frac{m_{b}}{\rho_{b}}\left(f_{a}-f_{b}\right) \frac{w_{a b}}{r_{a b}},
$$

where $w_{a b}$ is related to the kernel by $\nabla W_{a b}=\hat{e}_{a b} w_{a b}, \hat{e}_{a b}$ is the unit vector from particle $b$ to particle $a, \hat{e}_{a b}=\vec{r}_{a b} / r_{a b}$ and $\vec{r}_{a b}=\vec{r}_{a}-\vec{r}_{b}$. For more information on higher order derivatives we refer to the existing literature $[57,58,155,6]$. 


\subsubsection{The kernel function}

To restrict the number of contributing particles in the sum of Eq. (12) to a local subset, the kernel should have compact support, otherwise the summation would extend over all $n$ particles and produce a numerically intractable, completely inefficient $n^{2}$-method. Although for geometries such as flattened disks non-radial kernels seem like a natural choice [156-158] they have the disadvantage that it is difficult to ensure exact angular momentum conservation, see below. Therefore, usually radial kernels with $W\left(\vec{r}-\vec{r}^{\prime}, h\right)=W\left(\left|\vec{r}-\vec{r}^{\prime}\right|, h\right)$, are used in SPH.

The accuracy of the kernel interpolation is in practice rather difficult to quantify unless strongly simplifying assumptions about the particle distribution are made, which are usually not met in reality. One can expand $f\left(\overrightarrow{r^{\prime}}\right)$ in the integral approximant, Eq. (9), into a Taylor series around $\vec{r}$

$$
f\left(\overrightarrow{r^{\prime}}\right)=\sum_{k=0}^{\infty} \frac{(-1)^{k} h^{k} f^{(k)}(\vec{r})}{k !}\left(\frac{\vec{r}-\vec{r}^{\prime}}{h}\right)^{k} .
$$

By explicitly writing down the integrals over the different terms in the Taylor expansion on the RHS and comparing to the LHS, one can determine how well the approximation agrees with the original function. By requiring that error terms vanish, one can construct kernels of the desired order. In practice, however, such kernels may have unwanted properties such as negative values in certain regions and this may, in extreme cases, lead to unphysical (negative!) density estimates from Eq. (13). Although substantial work has been invested into constructing more accurate kernels $[159,160,105,161]$, none has been found to overall perform substantially better in practice than the "standard" cubic spline SPH kernel [5] which is used in almost all SPH simulations. Particular higher-order kernels, however, may have advantages in the context of resolving Kelvin-Helmholtz instabilities near large density gradients [162]. In 3D, the cubic spline kernel reads

$$
W(q)=\frac{1}{\pi h^{3}}\left\{\begin{array}{cc}
1-\frac{3}{2} q^{2}+\frac{3}{4} q^{3} & \text { for } 0 \leq q \leq 1 \\
\frac{1}{4}(2-q)^{3} & \text { for } 1<q \leq 2 \\
0 & \text { for } q>2
\end{array}\right.
$$

where $q=|\vec{r}-\vec{r}| / h$. This kernel is radial, i.e. it depends only on the absolute value of $\vec{r}-\vec{r}^{\prime}$. With this kernel the integral approximation, Eq. (9), is

$$
\tilde{f}_{h}(\vec{r})=f(\vec{r})+C h^{2}+O\left(h^{4}\right),
$$


where the quantity $C$ contains the second derivatives of the function $f$. Therefore, constant and linear functions are reproduced exactly by the integral representation Eq. (9).

In practice, two approximations were applied: i) the integral interpolation, Eq. (9) and ii) the discretization, Eq. (12). The accuracy of the discretized equations depends on the distribution of the interpolation points ("particles"). In early work, error estimates were based on a purely statistical description assuming a random distribution of particles. In a simulation, however, the particle distribution is not random, but depends on both the kernel used and the dynamics of the considered system. For this reason, numerical experiments turned out to be far more accurate than predicted by these early estimates [6].

In the following box we collect some expressions for the derivatives of radial kernels that are frequently used throughout this text.

\subsection{The "vanilla ice" SPH equations}

\subsubsection{Momentum equation}

One could now proceed according to "discretize and hope for the best" and this was historically the first approach. A straightforward discretization of Eq. (4) (no body forces) yields

$$
\frac{d \vec{v}_{a}}{d t}=-\frac{1}{\rho_{a}} \sum_{b} \frac{m_{b}}{\rho_{b}} P_{b} \nabla_{a} W_{a b}
$$

This form solves the Euler equation to the order of the method, but it does not conserve momentum. To see this, consider the force that particle $b$ exerts on particle a,

$$
\vec{F}_{b a}=\left(m_{a} \frac{d \vec{v}_{a}}{d t}\right)_{b}=-\frac{m_{a}}{\rho_{a}} \frac{m_{b}}{\rho_{b}} P_{b} \nabla_{a} W_{a b}
$$

and similarly from $a$ on $b$

$$
\vec{F}_{a b}=\left(m_{b} \frac{d \vec{v}_{b}}{d t}\right)_{a}=-\frac{m_{b}}{\rho_{b}} \frac{m_{a}}{\rho_{a}} P_{a} \nabla_{b} W_{b a}=\frac{m_{a}}{\rho_{a}} \frac{m_{b}}{\rho_{b}} P_{a} \nabla_{a} W_{a b}
$$

where Eq. (26) was used. Since in general $P_{a} \neq P_{b}$, this momentum equation does not fulfill Newton's third law ("actio = reactio") by construction and therefore total momentum is not conserved. 


\section{Derivatives of radial kernels}

Throughout the text, we use the notation $\vec{r}_{b k}=\vec{r}_{b}-\vec{r}_{k}, r_{b k}=\left|\vec{r}_{b k}\right|$ and $\vec{v}_{b k}=\vec{v}_{b}-\vec{v}_{k}$. Derivatives resulting from the smoothing lengths are ignored for the moment, they will receive particular attention in Sec. 3. By straight-forward component-wise differentiation one finds

$$
\frac{\partial}{\partial \vec{r}_{a}}\left|\vec{r}_{b}-\vec{r}_{k}\right|=\frac{\left(\vec{r}_{b}-\vec{r}_{k}\right)\left(\delta_{b a}-\delta_{k a}\right)}{\left|\vec{r}_{b}-\vec{r}_{k}\right|}=\hat{e}_{b k}\left(\delta_{b a}-\delta_{k a}\right),
$$

where $\hat{e}_{b k}$ is the unit vector from particle $k$ to particle $b$,

$$
\frac{\partial}{\partial \vec{r}_{a}} \frac{1}{\left|\vec{r}_{b}-\vec{r}_{k}\right|}=-\frac{\hat{e}_{b k}\left(\delta_{b a}-\delta_{k a}\right)}{\left|\vec{r}_{b}-\vec{r}_{k}\right|^{2}}
$$

and $\delta_{i j}$ is the usual Kronecker symbol. Another frequently needed expression is

$$
\begin{aligned}
\frac{d r_{a b}}{d t} & =\frac{\partial r_{a b}}{\partial x_{a}} \frac{d x_{a}}{d t}+\frac{\partial r_{a b}}{\partial y_{a}} \frac{d y_{a}}{d t}+\frac{\partial r_{a b}}{\partial z_{a}} \frac{d z_{a}}{d t}+\frac{\partial r_{a b}}{\partial x_{b}} \frac{d x_{b}}{d t}+\frac{\partial r_{a b}}{\partial y_{b}} \frac{d y_{b}}{d t}+\frac{\partial r_{a b}}{\partial z_{b}} \frac{d z_{b}}{d t} \\
& =\nabla_{a} r_{a b} \cdot \vec{v}_{a}+\nabla_{b} r_{a b} \cdot \vec{v}_{b}=\nabla_{a} r_{a b} \cdot \vec{v}_{a}-\nabla_{a} r_{a b} \cdot \vec{v}_{b}=\nabla_{a} r_{a b} \cdot \vec{v}_{a b}=\hat{e}_{a b} \cdot \vec{v}_{a b}
\end{aligned}
$$

where $\partial r_{a b} / \partial x_{b}=-\partial r_{a b} / \partial x_{a}$ etc. was used. For kernels that only depend on the magnitude of the separation, $W\left(\vec{r}_{b}-\vec{r}_{k}\right)=W\left(\left|\vec{r}_{b}-\vec{r}_{k}\right|\right) \equiv W_{b k}$, the derivative with respect to the coordinate of an arbitrary particle $a$ is

$$
\nabla_{a} W_{b k}=\frac{\partial}{\partial \vec{r}_{a}} W_{b k}=\frac{\partial W_{b k}}{\partial r_{b k}} \frac{\partial r_{b k}}{\partial \vec{r}_{a}}=\frac{\partial W_{b k}}{\partial r_{b k}} \hat{e}_{b k}\left(\delta_{b a}-\delta_{k a}\right)=\nabla_{b} W_{k b}\left(\delta_{b a}-\delta_{k a}\right),
$$

where Eq. (22) was used. This yields in particular the important property

$$
\nabla_{a} W_{a b}=\frac{\partial}{\partial \vec{r}_{a}} W_{a b}=\frac{\partial W_{a b}}{\partial r_{a b}} \frac{\partial r_{a b}}{\partial \vec{r}_{a}}=\frac{\partial W_{a b}}{\partial r_{a b}} \hat{e}_{a b}=-\frac{\partial W_{a b}}{\partial r_{a b}} \frac{\partial r_{a b}}{\partial \vec{r}_{b}}=-\frac{\partial}{\partial \vec{r}_{b}} W_{a b}=-\nabla_{b} W_{a b}
$$

The time derivative of the kernel is given by

$$
\frac{d W_{a b}}{d t}=\frac{\partial W_{a b}}{\partial r_{a b}} \frac{d r_{a b}}{d t}=\frac{\partial W_{a b}}{\partial r_{a b}} \frac{\left(\vec{r}_{a}-\vec{r}_{b}\right) \cdot\left(\vec{v}_{a}-\vec{v}_{b}\right)}{r_{a b}}=\frac{\partial W_{a b}}{\partial r_{a b}} \hat{e}_{a b} \cdot \vec{v}_{a b}=\vec{v}_{a b} \cdot \nabla_{a} W_{a b}
$$

But a slightly more sophisticated approach yields built-in conservation. If one starts from

$$
\nabla\left(\frac{P}{\rho}\right)=\frac{\nabla P}{\rho}-P \frac{\nabla \rho}{\rho^{2}}
$$

solves for $\nabla P / \rho$ and applies the gradient formula (14), the momentum equation reads 


$$
\begin{aligned}
\frac{d \vec{v}_{a}}{d t} & =-\frac{P_{a}}{\rho_{a}^{2}} \sum_{b} m_{b} \nabla_{a} W_{a b}-\sum_{b} \frac{m_{b}}{\rho_{b}} \frac{P_{b}}{\rho_{b}} \nabla_{a} W_{a b} \\
& =-\sum_{b} m_{b}\left(\frac{P_{a}}{\rho_{a}^{2}}+\frac{P_{b}}{\rho_{b}^{2}}\right) \nabla_{a} W_{a b} .
\end{aligned}
$$

Because the pressure part of the equation is now manifestly symmetric in $a$ and $b$ and $\nabla_{a} W_{a b}=-\nabla_{b} W_{b a}$, the forces are now equal and opposite and therefore total momentum and angular momentum (see below) are conserved by construction. It is worth pointing out some issues:

- So far, any complications resulting from variable smoothing lengths have been ignored. Conservation is guaranteed if a) constant smoothing lengths are used (which is a bad idea in practice!) or b) " $\nabla_{a} W_{a b}$ " is symmetric in the smoothing lengths, which can be achieved, for example, by using $h_{a b}=\left(h_{a}+\right.$ $\left.h_{b}\right) / 2[4]$ or by replacing the gradient via $\left[\nabla_{a} W\left(r_{a b}, h_{a}\right)+\nabla_{a} W\left(r_{a b}, h_{b}\right)\right] / 2$ [3].

- The conservation relies on the force being a term symmetric in $a$ and $b$ times $\hat{e}_{a b}$ (keep in mind that $\nabla_{a} W_{a b} \propto \hat{e}_{a b}$, see Eq. (26)).

- SPH's success largely relies on its excellent conservation properties which is guaranteed by the correct symmetry in the particle indices.

\subsubsection{Energy equation}

A suitable energy equation can be constructed from Eq. (5) in a straight forward manner:

$$
\frac{d u_{a}}{d t}=\frac{P_{a}}{\rho_{a}^{2}} \frac{d \rho_{a}}{d t}=\frac{P_{a}}{\rho_{a}^{2}} \frac{d}{d t}\left(\sum_{b} m_{b} W_{a b}\right)=\frac{P_{a}}{\rho_{a}^{2}} \sum_{b} m_{b} \vec{v}_{a b} \cdot \nabla_{a} W_{a b}
$$

where Eq. (27) was used. Together with an equation of state, the equations (13), (29) and (30) form a complete set of SPH equations.

For later use we also note that the velocity divergence can be conveniently expressed by using Eqs. (3) and (27) as

$$
(\nabla \cdot \vec{v})_{a}=-\frac{1}{\rho_{a}} \frac{d \rho_{a}}{d t}=-\frac{1}{\rho_{a}} \sum_{b} m_{b} \vec{v}_{a b} \cdot \nabla_{a} W_{a b}
$$

One can derive an alternative energy equation, and this will allow a smoother transition to the relativistic equations, by using the specific "thermokinetic" energy $\hat{e}_{a}=u_{a}+\frac{1}{2} v_{a}^{2}$ instead of the specific thermal energy $u_{a}$. The corresponding continuous evolution equation,

$$
\frac{d \hat{e}}{d t}=-\frac{1}{\rho} \nabla \cdot(P \vec{v})
$$


can be written as

$$
\frac{d \hat{e}}{d t}=-\frac{P}{\rho^{2}} \nabla \cdot(\rho \vec{v})-\vec{v} \cdot \nabla\left(\frac{P}{\rho}\right) .
$$

Applying Eq. (14) yields

$$
\begin{aligned}
\frac{d \hat{e}_{a}}{d t} & =-\frac{P_{a}}{\rho_{a}^{2}} \sum_{b} \frac{m_{b}}{\rho_{b}} \rho_{b} \vec{v}_{b} \cdot \nabla_{a} W_{a b}-\vec{v}_{a} \cdot \sum_{b} \frac{m_{b}}{\rho_{b}} \frac{P_{b}}{\rho_{b}} \nabla_{a} W_{a b} \\
& =-\sum_{b} m_{b}\left(\frac{P_{a} \vec{v}_{b}}{\rho_{a}^{2}}+\frac{P_{b} \vec{v}_{a}}{\rho_{b}^{2}}\right) \cdot \nabla_{a} W_{a b} .
\end{aligned}
$$

As we will see later, see Eq. (167), this is similar to the relativistic form of the energy equation.

\subsection{Conservation properties}

We can easily check the numerical conservation of the physically conserved quantities. The change of the total particle momentum is

$$
\begin{aligned}
\sum_{a} m_{a} \frac{d \vec{v}_{a}}{d t} & =\sum_{a} \sum_{b} \vec{F}_{b a}=\frac{1}{2}\left(\sum_{a} \sum_{b} \vec{F}_{b a}+\sum_{a} \sum_{b} \vec{F}_{b a}\right) \\
& =\frac{1}{2}\left(\sum_{a, b} \vec{F}_{a b}+\sum_{b, a} \vec{F}_{b a}\right)=\frac{1}{2}\left(\sum_{a, b}\left(\vec{F}_{a b}+\vec{F}_{b a}\right)\right)=0
\end{aligned}
$$

where the (dummy) summation indices were relabeled after the third equal sign and the notation from Eq. (20) and $\vec{F}_{a b}=-\vec{F}_{b a}$ were used.

The proof for angular momentum is analogous. The torque on particle $a$ is

$$
\vec{M}_{a}=\vec{r}_{a} \times \vec{F}_{a}=\vec{r}_{a} \times\left(m_{a} \frac{d \vec{v}_{a}}{d t}\right)=\vec{r}_{a} \times \sum_{b} \vec{F}_{b a}
$$

and thus

$$
\begin{aligned}
\frac{d \vec{L}}{d t} & =\sum_{a} \vec{M}_{a}=\sum_{a, b} \vec{r}_{a} \times \vec{F}_{b a}=\frac{1}{2}\left(\sum_{a, b} \vec{r}_{a} \times \vec{F}_{b a}+\sum_{a, b} \vec{r}_{a} \times \vec{F}_{b a}\right) \\
& =\frac{1}{2}\left(\sum_{a, b} \vec{r}_{a} \times \vec{F}_{b a}+\sum_{b, a} \vec{r}_{b} \times \vec{F}_{a b}\right)=\frac{1}{2}\left(\sum_{a, b}\left(\vec{r}_{a}-\vec{r}_{b}\right) \times \vec{F}_{b a}\right)=0 .
\end{aligned}
$$


Again, the dummy indices were relabeled and $\vec{F}_{a b}=-\vec{F}_{b a}$ was used. The expression finally vanishes, because the forces between particles act along the line joining them: $\vec{F}_{a b} \propto \nabla_{a} W_{a b} \propto \hat{e}_{a b} \propto\left(\vec{r}_{a}-\vec{r}_{b}\right)$, see Eq. (26).

The total energy changes according to

$$
\frac{d E}{d t}=\frac{d}{d t} \sum_{a}\left(m_{a} u_{a}+\frac{1}{2} m_{a} v_{a}^{2}\right)=\sum_{a} m_{a} \frac{d \hat{\epsilon}_{a}}{d t}=\sum_{a} m_{a}\left(\frac{d u_{a}}{d t}+\vec{v}_{a} \cdot \frac{d \vec{v}_{a}}{d t}\right) .
$$

Inserting Eqs. (30) and (29) we have

$$
\begin{aligned}
\frac{d E}{d t}= & \sum_{a} m_{a}\left[\frac{P_{a}}{\rho_{a}^{2}} \sum_{b} m_{b} \vec{v}_{a b} \cdot \nabla_{a} W_{a b}-\vec{v}_{a} \cdot \sum_{b} m_{b}\left(\frac{P_{a}}{\rho_{a}^{2}}+\frac{P_{b}}{\rho_{b}^{2}}\right) \nabla_{a} W_{a b}\right] \\
= & \sum_{a, b} m_{a} m_{b} \frac{P_{a}}{\rho_{a}^{2}} \vec{v}_{a} \cdot \nabla_{a} W_{a b}-\sum_{a, b} m_{a} m_{b} \frac{P_{a}}{\rho_{a}^{2}} \vec{v}_{b} \cdot \nabla_{a} W_{a b} \\
& -\sum_{a, b} m_{a} m_{b} \frac{P_{a}}{\rho_{a}^{2}} \vec{v}_{a} \cdot \nabla_{a} W_{a b}-\sum_{a, b} m_{a} m_{b} \frac{P_{b}}{\rho_{b}^{2}} \vec{v}_{a} \cdot \nabla_{a} W_{a b} \\
= & -\sum_{a, b} m_{a} m_{b}\left(\frac{P_{a} \vec{v}_{b}}{\rho_{a}^{2}}+\frac{P_{b} \vec{v}_{a}}{\rho_{b}^{2}}\right) \cdot \nabla_{a} W_{a b} .
\end{aligned}
$$

From this equation we could have directly read off the evolution equation for the specific thermokinetic energy, Eq. (34). To show the conservation of the total energy we can apply the same procedure as in the case of the momentum equation since we have again a double sum over a quantity symmetric in the indices $a, b$ multiplied by a quantity which is anti-symmetric in the indices $a, b$. Therefore, the double sum vanishes and total energy is conserved.

\subsection{Alternative SPH discretizations}

Like other numerical schemes, SPH offers some freedom in discretizing the continuum equations. At this point it is worth remembering that we had enforced momentum conservation by using a particular form of the gradient on the

RHS of the momentum equation, see Eq. (28). Following the same reasoning one could as well have used [5]

$$
\frac{\nabla P}{\rho}=\frac{P}{\rho^{\lambda}} \nabla\left(\frac{1}{\rho^{1-\lambda}}\right)+\frac{1}{\rho^{2-\lambda}} \nabla\left(\frac{P}{\rho^{\lambda-1}}\right)
$$

which yields

$$
\frac{d \vec{v}_{a}}{d t}=-\sum_{b} m_{b}\left(\frac{P_{a}}{\rho_{a}^{\lambda} \rho_{b}^{2-\lambda}}+\frac{P_{b}}{\rho_{a}^{2-\lambda} \rho_{b}^{\lambda}}\right) \nabla_{a} W_{a b}
$$


for the momentum equation. This form is symmetric in the particle indices for any value of $\lambda$ and therefore also conserves momentum by construction. This equation is consistent with the energy equation

$$
\frac{d u_{a}}{d t}=\frac{P_{a}}{\rho_{a}^{\lambda}} \sum_{b} m_{b} \frac{\vec{v}_{a b}}{\rho_{b}^{2-\lambda}} \cdot \nabla_{a} W_{a b}
$$

and the continuity equation

$$
\frac{d \rho_{a}}{d t}=\rho_{a}^{2-\lambda} \sum_{b} m_{b} \frac{\vec{v}_{a b}}{\rho_{b}^{2-\lambda}} \cdot \nabla_{a} W_{a b},
$$

which is the SPH-discrete form of

$$
\frac{d \rho_{a}}{d t}=\rho^{2-\lambda}\left[\vec{v} \cdot \nabla\left(\rho^{\lambda-1}\right)-\nabla \cdot\left(\vec{v} \rho^{\lambda-1}\right)\right] .
$$

The consistency of the above SPH equation set can be shown by using a generalized variational principle [105] that does not assume that the density is given as a function of the coordinates, but instead assumes a given form of the continuity equation.

If $\lambda=1$, the continuity equation only depends on the particle volumes $m_{b} / \rho_{b}$ rather than the particle masses. This can reduce numerical noise in regions of large density contrasts where particles of very different masses interact [163]. Marri and White [137] found improved results in their galaxy formation calculations by using (among other modifications) a value of $\lambda=3 / 2$.

The SPH equations can be generalized even further after noting that the continuity equation can be written as [105]

$$
\frac{d \rho}{d t}=\Psi\left\{\vec{v} \cdot \nabla\left(\frac{\rho}{\Psi}\right)-\nabla \cdot\left(\frac{\rho \vec{v}}{\Psi}\right)\right\}
$$

with $\Psi$ being a scalar variable defined on the particle field. This leads to SPH equations of the form

$$
\begin{aligned}
\frac{d \rho_{a}}{d t} & =\Psi_{a} \sum_{b} m_{b} \frac{\vec{v}_{a b}}{\Psi_{b}} \cdot \nabla_{a} W_{a b} \\
\frac{d \vec{v}_{a}}{d t} & =-\sum_{b} m_{b}\left(\frac{P_{a}}{\rho_{a}^{2}} \frac{\Psi_{a}}{\Psi_{b}}+\frac{P_{b}}{\rho_{b}^{2}} \frac{\Psi_{b}}{\Psi_{a}}\right) \nabla_{a} W_{a b} \\
\frac{d u_{a}}{d t} & =\frac{P_{a}}{\rho_{a}^{2}} \sum_{b} m_{b} \frac{\Psi_{a}}{\Psi_{b}} \vec{v}_{a b} \cdot \nabla_{a} W_{a b} .
\end{aligned}
$$


The "vanilla ice" equation set, Eqs. (29) and (30), is recovered for $\Psi=1$, choosing $\Psi=\rho / \sqrt{P}$ one recovers the momentum equation of Hernquist and Katz [3].

The above equation set has been used in recent work [162] to address SPH's weakness of resolving Kelvin-Helmholtz instabilities across large density jumps. The authors choose a different auxiliary function for each of the above equations, $\Psi^{\rho}, \Psi^{v}, \Psi^{u}$, and perform an error and stability analysis leading them to suggest $\Psi^{\rho}=\Psi^{u}=1 / A^{\Gamma}$ and $\Psi^{v}=\rho$. Here $A$ is the entropy-dependent part of the polytropic equation of state, $P=A(s) \rho^{\Gamma}$ and $\Gamma$ the polytropic exponent. This prescription leads to a sharper density transition that is more consistent with the entropy transitions between both parts of the fluid. Together with a higher-order kernel and large neighbor numbers they find convincing results in Kelvin-Helmholtz instability simulations.

\subsection{Adaptive resolution}

Whenever densities and length scales vary by large amounts, the smoothing length $h$ should be adapted in space and time. Several ways to adjust the smoothing lengths have been used over the years $[2,164,165,3,4,166]$. One of them seeks to keep the number of neighbors of each particle (approximately) constant [3], another to integrate an additional equation that makes use of the continuity equation [4]. One can use the ansatz

$$
\frac{h(t)}{h_{0}}=\left(\frac{\rho_{0}}{\rho(t)}\right)^{1 / 3}
$$

where the index 0 labels the quantities at the beginning of the simulation. Taking Lagrangian time derivatives of both sides together with Eq. (3) yields

$$
\frac{d h_{a}}{d t}=\frac{1}{3} h_{a}(\nabla \cdot \vec{v})_{a}
$$

Another convenient way is to evolve $h$ according to

$$
h_{a}=\eta\left(\frac{m_{a}}{\rho_{a}}\right)^{1 / 3}
$$

where $\eta$ should be chosen in the range between 1.2 and $1.5[165,105,6]$.

Note, that the above SPH equations were derived under the assumption that the smoothing lengths are constant. Varying the smoothing lengths while using the above equation set is strictly speaking inconsistent. A consistent formulation that involves extra-terms will be discussed in detail in Sec. 3.3. The 
importance of these corrective, or "grad-h" terms is problem- and resolutiondependent [146,112].

\subsection{Artificial dissipation}

\subsubsection{Purpose and general reasoning}

In gas dynamics, even perfectly smooth initial conditions can steepen into discontinuous solutions, or "shocks", see e.g. [167-169], which are nearly omnipresent in astrophysics. On length scales comparable to the gas mean free path these solutions are smooth (as a result of the physical viscosity that is always present to some extent), but on the macroscopic scales of a simulation (which is usually orders of magnitude larger), the very steep gradients appear as discontinuous.

The strategies to deal numerically with shocks are (broadly speaking) two-fold: i) one can either make use of the analytical solution of a Riemann problem between two adjacent computational entities (either cells or particles) or ii) broaden the discontinuity to a numerically resolvable length across which gradients can be calculated. The latter is instantiated by adding extra, "artificial" dissipation to the flow. This can be achieved by choosing particular numerical discretization schemes that in fact solve continuum equations that are only similar to the original ones, but contain additional, higher-order deviatoric terms, such as, for example, the Lax scheme [170]. Alternatively, one may explicitely add pressure-like terms to the fluid equations. The addition of such "ad-hoc" terms is one of the oldest techniques [171] in the relatively young field of computational physics. Most astrophysical SPH implementations follow the latter strategy, e.g. [3,14,12,112], but variants that use Riemann-solvers do also exist $[172,173]$.

Strictly speaking, real discontinuities with their infinite derivatives are no proper solutions of the ideal hydrodynamics equations Eqs. (3)-(5). Artificial viscosity $(\mathrm{AV})$ aims at spreading such discontinuities over a numerically resolvable length. In the words of von Neumann and Richtmyer (from their seminal paper [171]), the "idea is to introduce (artificial) dissipative terms into the equations so as to give the shocks a thickness comparable to (but preferentially larger than) the spacing ... [of the grid points]. Then the differential equations (more accurately, the corresponding difference equations) may be used for the entire calculation, just as though there were no shocks at all." Although guidance by physical viscosity can sometimes be helpful, artificial viscosity is not meant to mimic physical viscosity, it is instead an ad hoc method to produce on a resolvable scale what is the result of unresolvable, small-scale effects. In this sense one can think of it as a kind of sub-grid model. AV should have a number of desirable properties [174] and must not introduce unphysical artifacts. It should always be dissipative, i.e. transfer kinetic en- 
ergy into internal one and never vice versa, which is not a completely trivial task in 3D. AV should be absent in rigid and (shockless) differential rotation and in uniform compression. In other words, it should be "intelligent" in the sense that it distinguishes uniform compression from a shock. This can be done either via a tensor formulation of artificial viscosity, e.g. [175], or via "limiters" that detect such motion and subsequently suppress the action of AV. Generally, AV should go smoothly to zero as the compression vanishes and it should be absent for expansion. Moreover, to not destroy one of SPH's most salient strength, it must be implemented consistently into momentum and energy equation, so that the conservation of energy, momentum and angular momentum is still guaranteed.

\subsubsection{Bulk and von-Neumann-Richtmyer viscosity}

Guided by the requirements that i) no real discontinuities occur, ii) the thickness of the "shock layer" is everywhere of the order of the resolvable length scale $l$, iii) no noticeable effects occur away from shock layers and iv) the Rankine-Hugoniot relations [167] hold when considering length scales that are large in comparison to the thickness of the shock layers, von Neumann and Richtmyer suggested an artificial pressure of the form

$$
q_{\mathrm{NR}}=c_{2} \rho l^{2}(\nabla \cdot \vec{v})^{2}
$$

where $c_{2}$ is a dimensionless parameter of order unity, to be added to the hydrodynamic pressure. Their approach yielded reasonably good results in strong shocks, yet it still allowed oscillations in the post-shock region to occur. To avoid them, usually an additional term [176] is introduced that vanishes less rapidly and has the form of a bulk viscosity [167]

$$
q_{\mathrm{b}}=-c_{1} \rho c_{\mathrm{s}} l(\nabla \cdot \vec{v}) .
$$

Here $c_{\mathrm{s}}$ is the sound velocity and $c_{1}$ is a parameter of order unity. Often, the two contributions are combined into an artificial viscous pressure,

$$
q_{\mathrm{visc}}=-c_{1} \rho c_{\mathrm{s}} l(\nabla \cdot \vec{v})+c_{2} \rho l^{2}(\nabla \cdot \vec{v})^{2} .
$$

The term $l(\nabla \cdot \vec{v})$ is thereby an estimate for the velocity jump between adjacent cells or particles.

\subsection{3 "Standard" SPH viscosity: reasoning and limitations}

A straight-forward approach to transform these ideas into an SPH artificial viscosity prescription would be to insert Eq. (31) into (54), to use particle 
properties for the densities and sound velocities and identify the resolution length scale $l$ with the smoothing length. Similar approaches have indeed been pursued [3], but we will focus here on an approach due to [177] that is probably the most wide-spread form of SPH artificial viscosity and therefore often referred to as the "standard viscosity".

Instead of just increasing the hydrodynamic pressures, $P_{a}$, by viscous contributions, $q_{\text {visc }}$, one augments the pressure terms in the momentum equation (29) by an artificial contribution $\Pi_{a b}$ :

$$
\left(\frac{P_{a}}{\rho_{a}^{2}}+\frac{P_{b}}{\rho_{b}^{2}}\right) \rightarrow\left(\frac{P_{a}}{\rho_{a}^{2}}+\frac{P_{b}}{\rho_{b}^{2}}+\Pi_{a b}\right)
$$

For simplicity, let us consider the bulk viscosity contribution first and restrict ourselves to $1 \mathrm{D}$. The bulk contribution to $\Pi_{a b}$ is then of the form $-c_{1} c_{\mathrm{S}}(h / \rho) \partial v / \partial x$ and a Taylor expansion of the velocity field, $v\left(x_{a}+\left(x_{b}-x_{a}\right)\right)$, yields

$$
\left(\frac{\partial v}{\partial x}\right)_{a}=\frac{v_{b}-v_{a}}{x_{b}-x_{a}}+O\left(\left(x_{b}-x_{a}\right)^{2}\right) .
$$

Inserting this approximation, replacing particle properties by their averages, $\bar{A}_{a b}=\left(A_{a}+A_{b}\right) / 2$, using the notation $x_{a b}=x_{a}-x_{b}$ and $v_{a b}=v_{a}-v_{b}$ and replacing the possibly diverging denominator,

$$
\frac{1}{x_{a b}} \rightarrow \frac{x_{a b}}{x_{a b}^{2}+\epsilon \bar{h}_{a b}^{2}}
$$

the SPH prescription for the bulk viscosity reads

$$
\Pi_{a b, \mathrm{bulk}}=\left\{\begin{array}{cc}
-c_{1} \frac{\bar{c}_{s, a b}}{\bar{\rho}_{a b}} \mu_{a b} & \text { for } x_{a b} v_{a b}<0 \\
0 & \text { otherwise }
\end{array}, \text { where } \mu_{a b}=\frac{\bar{h}_{a b} x_{a b} v_{a b}}{x_{a b}^{2}+\epsilon \bar{h}_{a b}^{2}} .\right.
$$

The product $x_{a b} v_{a b}$ thereby detects whether particles are approaching $(<0)$ and only in this case artificial viscosity becomes active. The quantity $\mu_{a b}$ has taken over the role of the term $l(\nabla \cdot \vec{v})$ in the original prescription of von Neumann and Richtmyer. Having said this, we can now treat the von Neumann-Richtmyer term, $q_{\mathrm{NR}}$, in exactly the same way. Adapting to the usual SPH notation, $c_{1} \rightarrow \alpha, c_{2} \rightarrow \beta$, the artificial viscosity term reads

$$
\Pi_{a b}=\Pi_{a b, \mathrm{bulk}}+\Pi_{a b, \mathrm{NR}}=\left\{\begin{array}{cl}
\frac{-\alpha \bar{c}_{a b} \mu_{a b}+\beta \mu_{a b}^{2}}{\bar{\rho}_{a b}} & \text { for } \vec{r}_{a b} \cdot \vec{v}_{a b}<0 \\
0 & \text { otherwise }
\end{array}\right.
$$


Fig. 1. Schematic illustration of the shock tube problem.

with

$$
\mu_{a b}=\frac{\bar{h}_{a b} \vec{r}_{a b} \cdot \vec{v}_{a b}}{r_{a b}^{2}+\epsilon \bar{h}_{a b}^{2}}
$$

Note that the scalar quantities have now been replaced by vector quantities for the use in $3 \mathrm{D}$. Numerical experiments suggest the following values for the involved parameters: $\alpha \approx 1, \beta \approx 2$ and $\epsilon \approx 0.01$.

Accounting for artificial viscosity, the momentum equation reads

$$
\frac{d \vec{v}_{a}}{d t}=-\sum_{b} m_{b}\left(\frac{P_{a}}{\rho_{a}^{2}}+\frac{P_{b}}{\rho_{b}^{2}}+\Pi_{a b}\right) \nabla_{a} W_{a b} .
$$

To have a consistent formulation, the energy equation must be modified according to

$$
\frac{d u_{a}}{d t}=\frac{P_{a}}{\rho_{a}^{2}} \sum_{b} m_{b} \vec{v}_{a b} \cdot \nabla_{a} W_{a b}+\frac{1}{2} \sum_{b} m_{b} \Pi_{a b} \vec{v}_{a b} \cdot \nabla_{a} W_{a b}
$$

This combination still conserves energy, linear and angular momentum by construction, the proofs are analogous to the ones outlined above.

To illustrate the performance of this artificial viscosity prescription, let us consider a standard test case for hydrodynamic schemes, the so-called "Sod shocktube" [178] which is a particular realization of a Riemann problem. A tube filled with gas, such as illustrated in Fig. 1, contains a high density $\left(\rho_{1}=1\right) /$ high pressure $\left(p_{1}=1\right)$ region that is separated by a wall from a low density $\left(\rho_{2}=0.25\right) /$ low pressure $\left(p_{2}=0.1795\right)$ region. For the Sod test a polytropic equation of state with exponent $\Gamma=1.4$ is used. Once the wall is removed, the discontinuity decays into two elementary, non-linear waves that move in opposite directions: a shock moves into the unperturbed original low density region, while a rarefaction wave travels into the original high-density region. Between the shock and the tail of the rarefaction wave (the rightmost end of the rarefaction) two new states develop which are separated by a contact discontinuity across which the pressure is constant. The exact solution is sketched in Fig. 2.

If no artificial viscosity is applied in such a problem, strong post-shock oscillations occur as illustrated in Fig. 3 for the velocities in the Sod test. If the standard artificial viscosity prescription, Eq. (59) is used, the numerical 

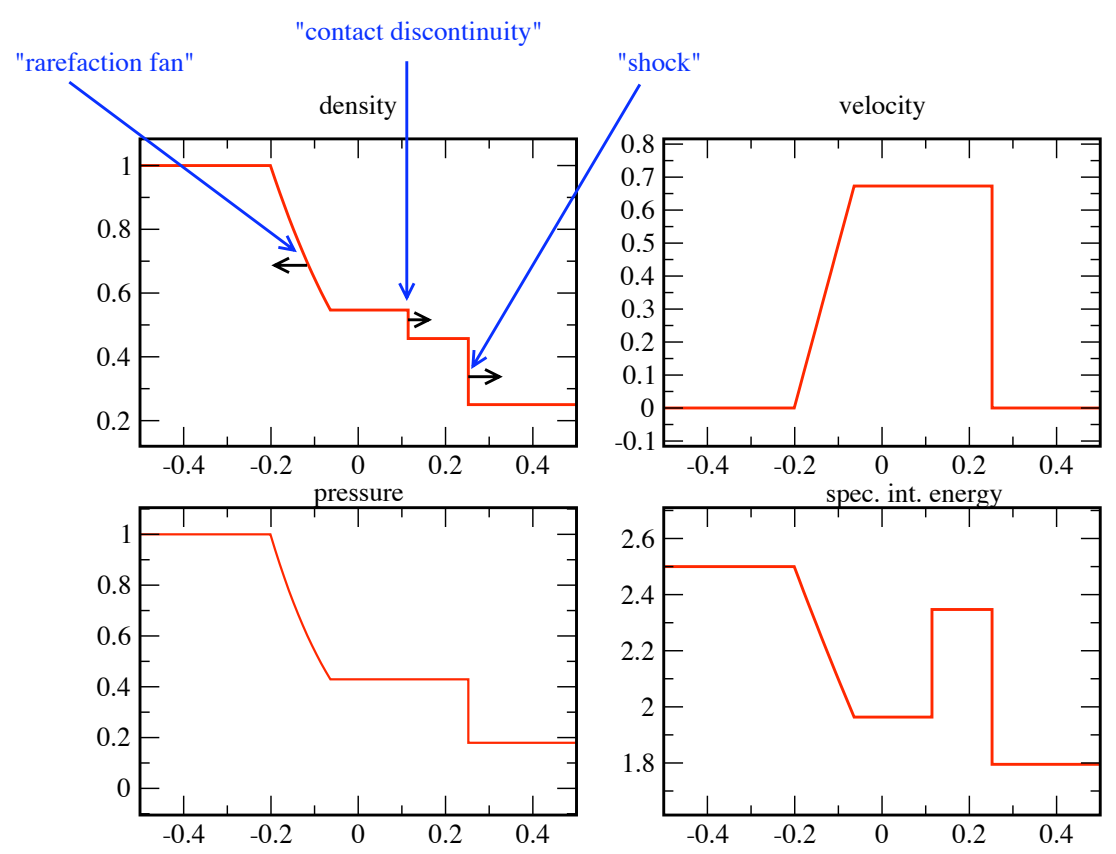

Fig. 2. The exact solution of the non-relativistic shock tube problem.

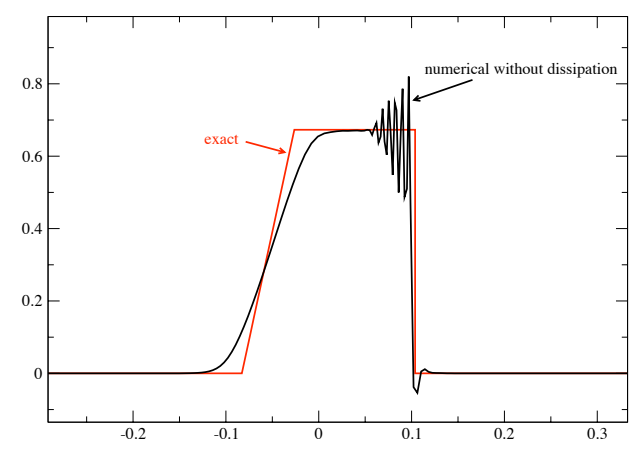

Fig. 3. Velocity snapshot of the Sod shock tube problem. Shown is the exact solution together with a numerical solution without artificial viscosity.

solution agrees very well with the exact one, see Fig. 4, provided that the interesting regions of the flow are adequately resolved ${ }^{3}$.

While this artificial viscosity form performs very well in 1D, it suffers from deficiencies if used in multi-D: i) for fixed parameters $\alpha$ and $\beta$ it may affect

3 Consistent with the finite resolution width of numerical shocks, we set up the initial density distribution as a Fermi function with a transition width of $\Delta x: \rho(x)=$ $\left(\rho_{1}-\rho_{2}\right) /\left(1+\exp \left(\frac{x-x_{S}}{\Delta x}\right)\right)+\rho_{2}$, where $x_{S}$ is the initial position of the shock. For the presented test $\Delta x$ was set to 1.5 times the average particle separation. 

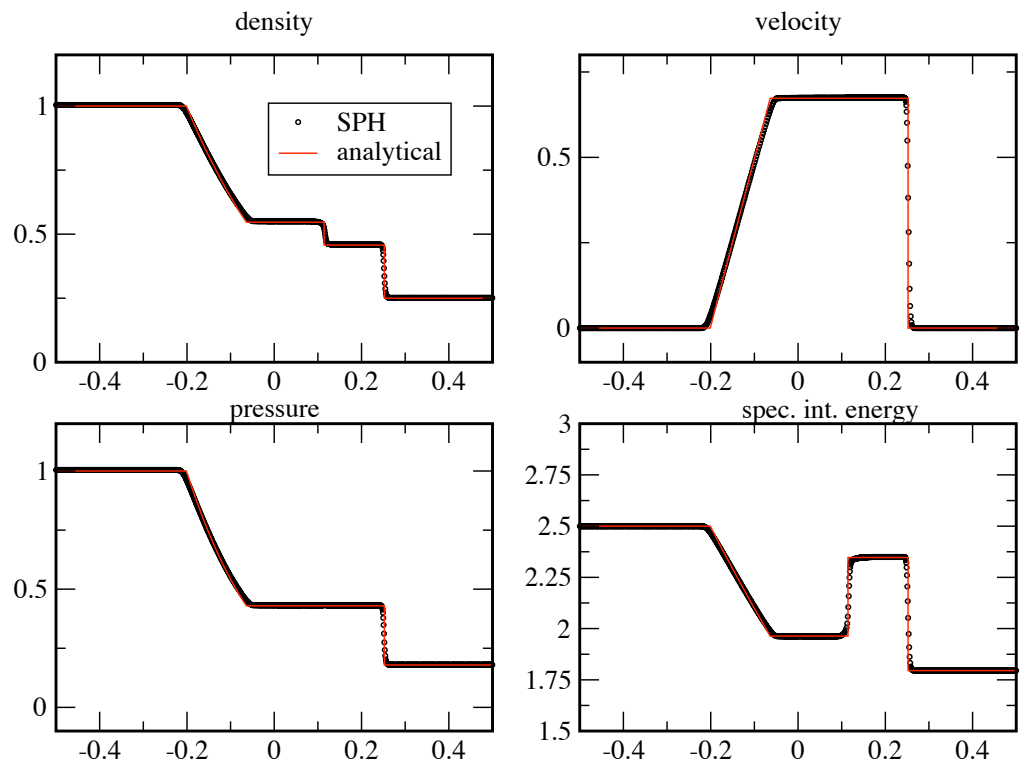

Fig. 4. Sod's shock tube: the exact solutions are shown by the red line, the numerical solution obtained with SPH are shown as circles. For this simulation 1000 particles were used, density was calculated via summation, and the smoothing lengths were updated according to $h_{a}=1.4\left(m_{a} / \rho_{a}\right)$. For this test a second-order Runge-Kutta integrator was used.

the flow even if it is not really needed and ii) under certain conditions it can introduce spurious shear forces. Consider for example an idealized shear flow as sketched in Fig. 5. Assume that the velocity decreases vertically as sketched on the left and no shocks occur. For such a situation no artificial viscosity is needed. Nevertheless, as sketched for two example particles ("1" and "2") the scalar product $\vec{r}_{a b} \cdot \vec{v}_{a b}$ is finite and thus, via $\mu_{a b}$ in Eq. (60), introduces a viscous force that is unwanted. Similar configurations are encountered for the (astrophysically important) cases of accretion disks.

\subsubsection{Reducing artificial viscosity where unnecessary}

Several recipes were suggested to cure the deficiencies that the standard artificial viscosity introduces in multi-D.

One such recipe is the so-called "Balsara-switch" [179]. Its strategy is to define a "limiter" that distinguishes shock from shear motion and suppresses AV in the latter case. One defines a quantity

$$
f_{a}=\frac{\left|\langle\nabla \cdot \vec{v}\rangle_{a}\right|}{\left|\langle\nabla \cdot \vec{v}\rangle_{a}\right|+\left|\langle\nabla \times \vec{v}\rangle_{a}\right|+0.0001 c_{s, a} / h_{a}}
$$




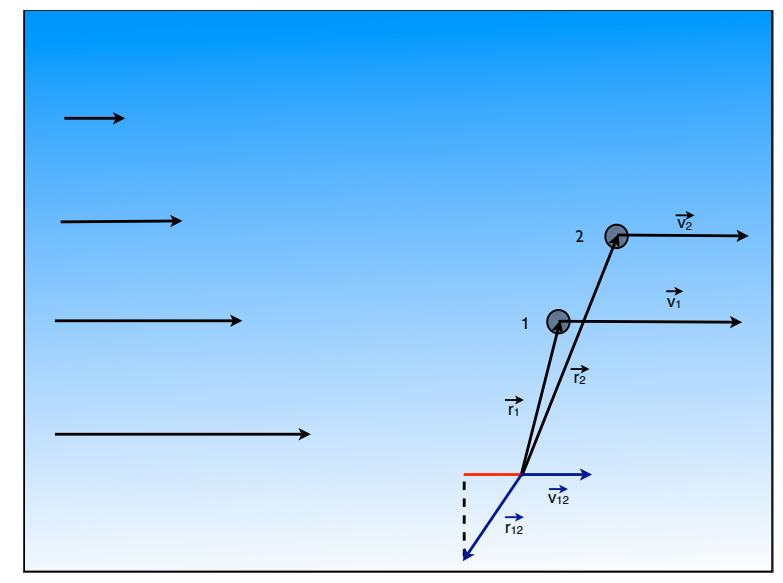

Fig. 5. Illustration how the "standard" SPH artificial viscosity introduces spurious shear forces: in this pure shear flow the difference position vector has a finite projection on the difference velocity vector (red) and thus introduces unwanted forces.

and, as before, applies a symmetrized average of the limiters $f, \Pi_{a b}^{\prime}=\Pi_{a b} \bar{f}_{a b}$. In pure compressional motion $(|\langle\nabla \cdot \vec{v}\rangle| \neq 0$ and $|\langle\nabla \times \vec{v}\rangle|=0)$ the limiter reduces to unity and the standard viscosity is recovered, and in pure shear flow $(|\langle\nabla \cdot \vec{v}\rangle|=0$ and $|\langle\nabla \times \vec{v}\rangle| \neq 0)$ the action of artificial viscosity is suppressed $\left(\bar{f}_{a b} \ll 1\right)$. This limiter has been found very useful in many cases $[149,180,181]$, but it reaches its limitations if shocks occur in a shearing environment such as in an accretion disk [175].

Morris and Monaghan [53] suggested the use of time-dependent artificial viscosity parameters. The main idea is to have $\alpha$ and $\beta$ only at non-negligible levels where they are really needed. They suggested to fix $\beta$ to $2 \alpha$, to assign each particle its individual parameter, $\alpha_{a}$, and to evolve it according to an additional differential equation,

$$
\frac{d \alpha_{a}}{d t}=-\frac{\alpha_{a}-\alpha_{\min }}{\tau_{a}}+S_{a}
$$

so that $\alpha_{a}$ decays exponentially with an e-folding time $\tau_{a}$ to a minimum value $\alpha_{\text {min }}$, unless it is triggered to rise by the source term $S_{a}$. The time scale $\tau_{a}$ should be chosen so that the viscosity persists for a few smoothing lengths behind the shock. This can be obtained by

$$
\tau_{a}=\frac{h_{a}}{\xi c_{s, a}},
$$

where $\xi \approx 0.1^{4}$. Morris and Monaghan suggested $S_{a}=\max \left[-(\vec{\nabla} \cdot \vec{v})_{a}, 0\right]$ for the source term. If one wishes to restrict the growth of $\alpha$ to $\alpha_{\max }$, one can use $S_{a}=\max \left[-(\vec{\nabla} \cdot \vec{v})_{a}\left(\alpha_{\max }-\alpha_{a}\right), 0\right][181]$. Typical values for the numerical

$\overline{4}$ Note the similarity to the SPH-version of the Courant time step criterion. 
parameters are $\alpha_{\min }=0.1$ and $\alpha_{\max }=1.5$. In a Sod test this prescription leads to non-negligible values of $\alpha_{a}$ only in the vicinity of the shock front, elsewhere artificial viscosity is practically absent. This prescription has largely removed unwanted effects from AV in three-dimensional SPH simulations [181,182]. Note, however, that in homologous flow, $\vec{v} \propto \vec{r}, \alpha_{a}$ can still rise although it is not needed. This is counterbalanced to some extent by the increase of $c_{s, a}$ and the decrease of $h_{a}$, so that -via $\tau_{a^{-}}$the decay term in Eq. (64) becomes more dominant, but there is certainly still room for improvement.

\subsubsection{New forms inspired by Riemann solvers}

Monaghan used the analogy to Riemann solvers to motivate a new form of artificial dissipation which involves signal velocities and jumps in variables across characteristics [183]. The main idea of these "discontinuity capturing terms" is that for any conserved scalar variable $A$ with $\sum_{a} m_{a} d A_{a} / d t=0$ a dissipative term of the form

$$
\left(\frac{d A_{a}}{d t}\right)_{\mathrm{diss}}=\sum_{b} m_{b} \frac{\alpha_{A, b} v_{\mathrm{sig}}}{\bar{\rho}_{a b}}\left(A_{a}-A_{b}\right) \hat{e}_{a b} \cdot \nabla_{a} W_{a b}
$$

should be added, where the parameter $\alpha_{A, b}$ determines the exact amount of dissipation and $v_{\text {sig }}$ is the maximum signal velocity between particle $a$ and $b$. Applied to velocity and thermokinetic energy this yields

$$
\begin{aligned}
& \left(\frac{d \vec{v}_{a}}{d t}\right)_{\text {diss }}=\sum_{b} m_{b} \frac{\alpha v_{\text {sig }}\left(\vec{v}_{a}-\vec{v}_{b}\right) \cdot \hat{e}_{a b}}{\bar{\rho}_{a b}} \nabla_{a} W_{a b} \\
& \left(\frac{d \hat{e}_{a}}{d t}\right)_{\text {diss }}=\sum_{b} m_{b} \frac{e_{a}^{*}-e_{b}^{*}}{\bar{\rho}_{a b}} \hat{e}_{a b} \cdot \nabla_{a} W_{a b},
\end{aligned}
$$

where, following [154], the energy including velocity components along the line of sight between particles $a$ and $b, e_{a}^{*}=\frac{1}{2} \alpha v_{\text {sig }}\left(\vec{v}_{a} \cdot \hat{e}_{a b}\right)^{2}+\alpha_{u} v_{\text {sig }}^{u} u_{a}$, has been used and different signal velocities and dissipation parameters were explicitely allowed for. If one uses $d u_{a} / d t=d \hat{e}_{a} / d t-\vec{v}_{a} \cdot d \vec{v}_{a} / d t$, one finds the dissipative terms of the thermal energy equation

$$
\left(\frac{d u_{a}}{d t}\right)_{\mathrm{diss}}=-\sum_{b} \frac{m_{b}}{\bar{\rho}_{a b}}\left[\alpha v_{\mathrm{sig}} \frac{1}{2}\left(\vec{v}_{a b} \cdot \hat{e}_{a b}\right)^{2}+\alpha_{u} v_{\mathrm{sig}}^{u}\left(u_{a}-u_{b}\right)\right] \hat{e}_{a b} \cdot \nabla_{a} W_{a b}
$$

The first term in this equation bears similarities with the "standard" artificial viscosity prescription, see Eq. (62), the second one expresses the exchange of thermal energy between particles and therefore represents an artificial thermal conductivity which smoothes discontinuities in the specific energy. Such 
artificial conductivity had been suggested earlier to cure the so-called "wall heating problem" [184]. Tests have shown that artificial conductivity substantially improves SPH's performance in simulating Sedov blast waves [112].

For non-relativistic hydrodynamics the maximum signal velocity between two particles can be estimated as [183]

$$
v_{\mathrm{sig}}=c_{\mathrm{s}, a}+c_{\mathrm{s}, b}-\vec{v}_{a b} \cdot \hat{e}_{a b}
$$

where $c_{\mathrm{s}, k}$ is the sound velocity of particle $k$. Price [154] had realized that SPH's difficulty to treat Kelvin-Helmholtz instabilities across contact discontinuities with large density jumps [185] is closely related to a "blip" that occurs in the pressure at the contact discontinuities ${ }^{5}$. He suggested to use artificial conductivity only to eliminate spurious pressure gradients across contact discontinuities and to this end suggested

$$
v_{\mathrm{sig}}^{u}=\sqrt{\frac{\left|P_{a}-P_{b}\right|}{\bar{\rho}_{a b}}} .
$$

Clearly, this quantity has the dimensions of a velocity and vanishes in pressure equilibrium. This approach has substantially improved SPH's ability to treat Kelvin-Helmholtz instabilities [154].

To avoid conductivity where it is unwanted, one can follow again a strategy with time-dependent parameters. For the artificial viscosity one can use Eq. (64), and proceed in a similar way for $\alpha_{u}$. One can use the second derivative of the thermal energy,

$$
S_{u, a}=\frac{h_{a}\left|\nabla^{2} u\right|_{a}}{\sqrt{u_{a}+\epsilon}}
$$

to control the growth $\alpha_{u}$. The second derivative can be calculated as in Eq. (15), the parameter $\epsilon$ avoids that $S_{u, a}$ diverges as $u_{a} \rightarrow 0$.

According to a recent analysis [162], SPH's difficulty to treat Kelvin-Helmholtz instabilities results from a mismatch in the sharpness of pressure and density across the density jump. This can be either cured by generating entropy at the boundary and thus smoothing the pressure as in [154], or by obtaining a sharper density estimate. By a combination of using the freedom in discretization, see Sec. 2.5, a particular, higher-order kernel and an entropy-weighted density estimate together with large neighbor numbers, [162] also find convincing results Kelvin-Helmholtz instability simulations.

$\overline{5}$ It is not visible in our Sod shock tube in Fig. 4 since we had started from smoothed initial conditions. 


\subsection{Time integration in SPH}

To integrate the ordinary differential equations of SPH one has to find a reasonable tradeoff between accuracy and efficiency of an integrator and most often the available computer power is better invested in larger particle numbers rather than in high-order integration schemes. Since the evaluation of derivatives is usually very expensive, and in particular so for self-gravitating fluids, one tries to minimize the number of force evaluations per time step, which gives preference to low-order integrators. If the storage of derivatives (from earlier time steps) is not a concern, one can resort to multi-step methods such as Adams-Bashforth-type integrators, e.g. [170,186], giving higher-order time integration accuracy at moderate costs of force evaluations.

After a short collection of commonly used time step criteria, we want to discuss briefly two methods that we find particularly useful: the Störmer-Verlet/leap frog algorithm, which appeals by its conservation properties and the class of Fehlberg methods whose advantage is the appropriate choice of the time step size based on monitoring the quality of the numerical solution.

\subsubsection{Time stepping}

We will briefly collect here commonly used time step criteria, depending on the considered physical system, further criteria that capture the specific physical time scales have to be added. A criterion that triggers on accelerations, $\vec{a}_{a}$, is $[5]$

$$
\Delta t_{f, a} \propto \sqrt{h_{a} /\left|\vec{a}_{a}\right|}
$$

and

$$
\Delta t_{C V, a} \propto \frac{h_{a}}{v_{\mathrm{s}, a}+0.6\left(c_{\mathrm{s}, a}+2 \max _{b} \mu_{a b}\right)}
$$

is a combination of a Courant-type ${ }^{6}$ and viscous time step control, where $\mu_{a b}$ is the quantity defined in Eq. (60). If one wishes to restrict the change of the smoothing length over a time step, one can use additionally[151]

$$
\Delta t_{h, a} \propto \frac{h_{a}}{\dot{h}_{a}}
$$

6 The Courant or Courant-Friedrichs-Levi (or CFL for short) criterion ensures that the numerical propagation speed of information does not exceed the physical one. If a spatial scale of $\Delta x$ can be resolved, the numerical time step has to be $\Delta t<\Delta x / c_{\mathrm{s}}$ to ensure numerical stability[170]. 
In a simulation, the minimum of the different time step criteria (with a suitably chosen prefactor) determines the hydrodynamical time step. If further physical processes, say nuclear burning, occur on much shorter time scales, one may resort to an "operator splitting" approach and integrate different processes with separate integration schemes, e.g. [67].

For inexpensive test problems or cases where the physical time scales are (more or less) the same for each particle, it is easiest to evolve all the particles on the same (shortest particle) time step. In many astrophysical examples, say cosmological structure formation, the collapse of a molecular cloud or the tidal disruption of a star by a black hole, the required time steps in different parts of the fluid may span many orders of magnitude. In such cases it is beneficial to group particles into block time steps of $\Delta t_{n}=2^{n} \Delta t_{\min }$, where $n$ is an integer and $\Delta t_{\min }$ is the smallest required time step, and to evolve each group of particles separately. In this way the number of (expensive) force evaluations can be reduced by orders of magnitude. Successful implementations of such individual time step schemes can be found, for example, in [187,188,3,123,189,12,190,151].

\subsubsection{Störmer-Verlet and leap frog}

The Verlet or leapfrog algorithm ${ }^{7}$ is particularly appealing due to its exact time reversibility. The original Verlet algorithm [191] is very easy to derive: start from two Taylor expansions for $\vec{r}(t+\Delta t)$ and $\vec{r}(t-\Delta t)$, add them and solve for $\vec{r}(t+\Delta t)$ to find the position update prescription of the Verlet algorithm

$$
\vec{r}(t+\Delta t)=2 \vec{r}(t)-\vec{r}(t-\Delta t)+\vec{a}(t) \Delta t^{2}+O\left(\Delta t^{3}\right) .
$$

It is interesting to note that the position is updated without using the velocities. But this comes at a price: for a position update two positions at earlier time steps are needed. The velocity can be reconstructed from the positions via centered finite differences:

$$
\vec{v}(t)=\frac{\vec{r}(t+\Delta t)-\vec{r}(t-\Delta t)}{2 \Delta t}+O\left(\Delta t^{3}\right) .
$$

This simple integrator is only second-order accurate, but it is time reversible and has excellent conservation properties. To kick off a simulation at $t=0$, one needs $\vec{r}(-\Delta t)$. It can be obtained by solving for $\vec{r}(-\Delta t)$ after inserting $t=0$ into Eq. (77):

$$
\vec{r}(-\Delta t)=\vec{r}(\Delta t)-2 \Delta t \vec{v}_{0}
$$

7 This algorithm has many names: in astronomy it is often called Störmer method, in molecular dynamics it is usually named Verlet method in the context of partial differential equations it is usually referred to as leap-frog method.

Note that the scheme needs modification if the acceleration depends on the velocity. 
where $\vec{v}_{0}=\vec{v}(t=0)$. Inserting this into Eq. (76) provides the position update

$$
\vec{r}(\Delta t)=\vec{r}_{0}+\Delta t \vec{v}_{0}+\frac{1}{2} \vec{a}_{0} \Delta t^{2}
$$

which looks like the first terms of a Taylor expansion around $t=0$.

The "leapfrog form" is obtained by defining velocities at half-steps, again via centered differences

$$
\vec{v}\left(t-\frac{\Delta t}{2}\right)=\frac{\vec{r}(t)-\vec{r}(t-\Delta t)}{\Delta t} \text { and } \vec{v}\left(t+\frac{\Delta t}{2}\right)=\frac{\vec{r}(t+\Delta t)-\vec{r}(t)}{\Delta t} .
$$

The last equation can be solved for the leapfrog form of the position equation

$$
\vec{r}(t+\Delta t)=\vec{r}(t)+\Delta t \vec{v}\left(t+\frac{\Delta t}{2}\right)
$$

Starting from Eq. (76) and inserting Eq. (80) we find the velocity update of the leapfrog algorithm

$$
\vec{v}\left(t+\frac{\Delta t}{2}\right)=\vec{v}\left(t-\frac{\Delta t}{2}\right)+\vec{a}(t) \Delta t
$$

So positions and accelerations are always evaluated at "full" time steps $t^{n}$, while the velocities are evaluated in between, either at $t^{n-1 / 2}$ or $t^{n+1 / 2}$, so the velocities are always "leaping" over the positions. If velocities at $t$ are needed, they can be calculated according to

$$
\vec{v}(t)=\frac{\vec{v}\left(t+\frac{\Delta t}{2}\right)+\vec{v}\left(t-\frac{\Delta t}{2}\right)}{2} .
$$

The "Störmer-Verlet form" is particularly useful, since all quantities are evaluated at the same point of time. Start from Eq. (77) and solve for

$$
\vec{r}(t-\Delta t)=\vec{r}(t+\Delta t)-2 \Delta t \vec{v}(t)
$$

Insert this into the Verlet position update, Eq. (76), to find the position update of the velocity Störmer-Verlet algorithm

$$
\vec{r}(t+\Delta t)=\vec{r}(t)+\vec{v}(t) \Delta t+\frac{1}{2} \vec{a}(t) \Delta t^{2} .
$$

To find the velocity update for the algorithm start from Eq. (77) and insert the Verlet position update, Eq. (76), 


$$
\vec{v}(t)=\frac{\vec{r}(t)-\vec{r}(t-\Delta t)}{\Delta t}+\frac{\vec{a}(t)}{2} \Delta t
$$

Completely analogous one finds for the velocity at $t+\Delta t$

$$
\vec{v}(t+\Delta t)=\frac{\vec{r}(t+\Delta t)-\vec{r}(t)}{\Delta t}+\frac{\vec{a}(t+\Delta t)}{2} \Delta t
$$

Adding the last two equations and using Eq. (77) gives

$$
\vec{v}(t+\Delta t)=\vec{v}(t)+\Delta t \frac{\vec{a}(t)+\vec{a}(t+\Delta t)}{2} .
$$

Together with Eq. (85) this update forms the Störmer-Verlet algorithm. It is a simple example of a "geometric integrator" which preserves qualitative features of the exact flow of the ODE by construction[192,193]. In particular, it conserves angular momentum exactly ${ }^{8}$.

The integration of an elliptical Kepler orbit represents a simple, yet significant numerical experiment. As long as no deviations from purely Newtonian point mass gravity are considered, bound orbits are closed ellipses, e.g. [194,195], any "non-closure" is a numerical artifact due to finite integration accuracy. To define the problem, we use units in which a test body ("planet") is accelerated by

$$
\vec{a}=-\frac{\vec{r}}{r^{3}}
$$

and, since angular momentum is conserved in a central force field, one only needs to consider two spatial dimensions. We choose $\vec{r}_{0}=(1,0)$ and $\vec{v}_{0}=$ $(0,0.2)$ as initial conditions. We perform a simulation up to $t=150$ with a slightly too large time step, $\Delta t=10^{-3}$. For comparison we also use a fourthorder Runge-Kutta scheme with the same time step. The results are displayed in Fig. 6. Due to the inappropriate time step the numerical inaccuracies in the velocity Störmer-Verlet case lead to a perihelion shift, but otherwise the behavior is physical: the test particle orbits the center of gravity on an eccentric orbit. In the Runge-Kutta case a qualitatively different and unphysical effect occurs: energy and angular momentum are dissipated for purely numerical reasons. The evolution of angular momentum is shown in Fig. 7. The "steps" in the Runge-Kutta case occur at perihelion since here the time steps are much too large. Therefore, the test particle drifts into a more circular, lower energy orbit. This illustrates that exact conservation can be more important that high order!

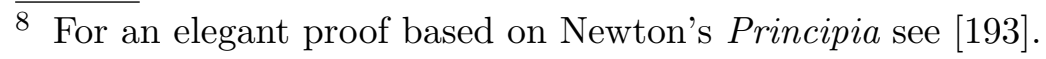



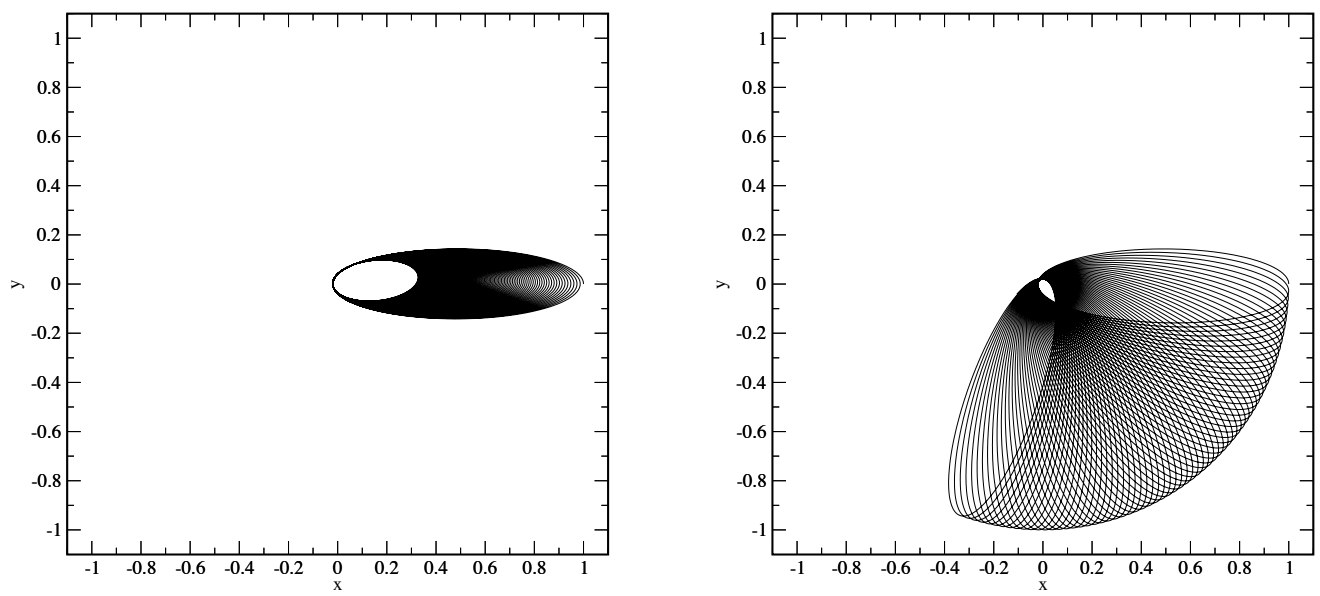

Fig. 6. Integration of the test particle orbit up to $t=150$ for an eccentric orbit $\left(\vec{v}_{0}=(0,0.2), \Delta t=10^{-3}\right)$. Left: fourth-order Runge-Kutta. Right: velocity Störmer-Verlet.

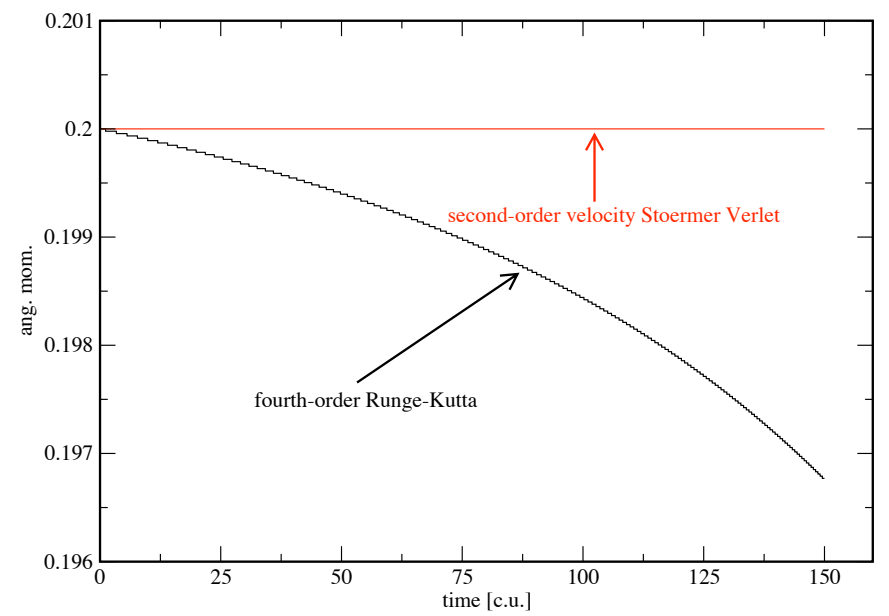

Fig. 7. Angular momentum evolution of the long-term comparison. While the velocity Störmer-Verlet method conserves angular momentum to machine precision, the fourth-order Runge-Kutta method loses about 2 percent. As a result of this purely numerical dissipation the planet spirals inward.

\subsubsection{Time stepping with quality control: Fehlberg methods}

The size of the time step $\Delta t$ is crucial for the accuracy (and stability!) of the numerical solution of an ODE. It has to be a small fraction of the shortest physical time scale of the problem at hand. For a complex multi-physics system this may require to determine the time scales of every involved physical process. In the worst case, these may not be known and, on top, the meaning 
of "a small fraction" may subject to trial and error.

Ideally, a method should decide itself which step size to take. This is the philosophy behind adaptive step size control or Fehlberg methods [196-198,170]. The main idea is to use two estimates of different order for the solution and use them to estimate the error growth rate. Based on the comparison of the estimated and the tolerable growth rate, the chosen time step may be accepted or rejected. Independent of whether the time step was accepted or not, the growth rate can provide an educated guess for the size of the next time step. The procedure is the following:

- Choose a trial time step $\Delta t$.

- Use two different methods to obtain results at the end of the time step, $a_{1}$ and $a_{2}$. To be reasonably efficient, the two methods should use nearly the same evaluations of the RHS of the ODE.

- Use $a_{1}$ and $a_{2}$ to calculate an approximate local truncation error $E$.

- Compare the error growth rate $\epsilon \approx E / \Delta t$ with some acceptable error growth rate, $\epsilon^{\text {acc }}$. If the rate is small enough, $\epsilon<\epsilon^{\text {acc }}$, accept the time step, $t^{n+1}=$ $t^{n}+\Delta t$ and $y^{n+1}=a_{2}$, where $a_{2}$ is the more accurate method. Otherwise, retake the step with a reduced step size determined from $\epsilon$ and the previously tried step size.

Obviously, the above strategy can be applied to a large variety of integrator combinations. An often used example is the combination of fourth- and fifth-order Runge-Kutta schemes [198]. As a low-order example, we apply the outlined strategy to a second- and a third-order method. Let $\Phi(t)$ be the exact solution of the differential equation and assume that an approximation to the solution at $t^{n}$ is known: $\Phi\left(t^{n}\right) \simeq y^{n}$. Take three RHS evaluations of the ODE:

$$
\begin{aligned}
& f_{1}=f\left(t^{n}, y^{n}\right) \\
& f_{2}=f\left(t^{n}+\Delta t, y^{n}+\Delta t f_{1}\right) \\
& f_{3}=f\left(t^{n}+\frac{\Delta t}{2}, y^{n}+\frac{\Delta t}{4}\left[f_{1}+f_{2}\right]\right)
\end{aligned}
$$

to produce a second-order (this is just the modified Euler method)

$$
a_{1}=y^{n}+\Delta t \frac{f_{1}+f_{2}}{2}
$$

and a third-order method

$$
a_{2}=y^{n}+\frac{\Delta t}{6}\left[f_{1}+f_{2}+4 f_{3}\right] .
$$

Thus, we have

$$
\Phi\left(t^{n}+\Delta t\right)=a_{1}+K \Delta t^{3}+O\left(\Delta t^{4}\right)=a_{2}+O\left(\Delta t^{4}\right) .
$$


Therefore, the leading error is

$$
E=\left|a_{2}-a_{1}\right|=K \Delta t^{3}+O\left(\Delta t^{4}\right)
$$

and the error grows at a rate

$$
\epsilon \approx \frac{E}{\Delta t} \approx K \Delta t^{2}
$$

The step can either be accepted $\left(\epsilon<\epsilon^{\text {acc }}\right), t^{n+1}=t^{n}+\Delta t, y^{n+1}=a_{2}$, or rejected $\left(\epsilon>\epsilon^{\text {acc }}\right)$ and re-taken. In both cases one can use the error measure to estimate the new time step. Since $\epsilon \propto \Delta t^{2}$, one finds

$$
\frac{\epsilon^{\text {new }}}{\epsilon^{\text {tried }}}=\left(\frac{\Delta t^{\text {new }}}{\Delta t^{\text {tried }}}\right)^{2}
$$

and the suggestion for the new time step is

$$
\Delta t^{\text {new }}=s\left(\frac{\epsilon^{\text {acc }}}{\epsilon^{\text {tried }}}\right)^{1 / 2} \Delta t^{\text {tried }}
$$

where $s<1$ is a "safety factor" for a conservative choice of the next time step. For further reading on ODE-integration the excellent text books of $[16,192,193,199]$ are recommended.

\section{9 "Best practice" suggestions}

We want to collect here a couple of suggestions born form practical experience that should help to carry out reliable simulations and to avoid numerical artifacts. This is somewhat "soft", yet hopefully still useful knowledge.

\section{- Neighbor numbers and resolution}

We recommend a large neighbor number, typically 100 or more. This somewhat increases the smoothing lengths and thus deteriorates the integral approximation, Eq. (18). On the other hand, it can substantially reduce numerical noise, i.e. fluctuations around the true solution. To compensate, it is advisable to run simulations at the highest affordable particle number, numerical resolution does matter. Substructures can only be considered resolved if they are substantially larger than the local smoothing lengths. If just an idea about the mass distribution is required, one may get away with a small particle number, say a few thousand particles, but reliable thermodynamic properties usually require much larger particle numbers. Contrary 
to some prejudices, SPH can resolve shocks properly, but since shocks are spread across a few smoothing lengths an appropriate numerical resolution is required.

\section{- Particle masses}

If at all possible, equal-mass particles should be used. Light particles that interact with much more massive ones easily become "nervous" ("ping pong and cannon ball effect"), i.e. they can exhibit large fluctuations. In some problems different particle numbers are unavoidable, if, for example, a highly centrally condensed star is considered, the Courant time step criterion may restrict the central time steps to prohibitively small values. In such cases one should make sure that interacting particles only differ in masses by factors of very few.

\section{- Initial conditions}

Accurate initial conditions are absolutely crucial for reliable simulations. The SPH particles should start out from their true numerical equilibrium positions. This can be obtained by applying an artificial damping term $\propto \vec{v}_{a}$ in the momentum equation that drags the particles to the desired initial conditions. Once equilibrium is found the particles should not move considerably during a few dynamical time scales after the damping has been switched off.

\section{- Time integration}

Ideally, an integration scheme should monitor the achieved accuracy for the tried time step and reject it if the error becomes unacceptably large. Just estimating a time step that seems reasonable and than hope for the best, may produce noisy, or in the worst case, spurious results. The rejection of time steps in individual time step schemes may, however, pose bookkeeping challenges. A simple workaround has been proposed by [200]. For each and every simulation the conservation of energy, momentum and angular momentum should be monitored. Reducing the time step size and increasing the force accuracy, say, if a tree is used for gravity, should improve the conservation properties. A correct code should ensure conservation to better than $1 \%$ over several thousand time steps.

\section{- Artificial dissipation}

Artificial dissipation should only be applied where really necessary. Schemes with time-dependent parameters are highly recommended. Modern versions of artificial dissipation terms such as Eqs. (67) and (68) should be used. Recent years have seen a large leap forward in eliminating unwanted effects from artificial dissipation, yet, there is certainly room for further improvement.

\section{- Displaying results}

Generally, display continuous quantities and avoid particle plots to present physical results. The particles are just auxiliary constructs, "moving interpolation points" that represent a continuum. Just showing particle positions projected on a plane may mimic a "resolution" that does not exist, a contour plot is a more honest presentation. To check that the numerics is well 
behaved, it may, however, be useful to inspect the particle distribution. A noisy particle distribution should always raise doubts about the quality of a simulation.

\section{Summary of "vanilla ice" SPH}

We summarize here the most basic form of the SPH equations. As the particle masses are kept fix, there is no need to solve the continuity equation. Densities can be obtained via summation from

$$
\rho_{a}=\sum_{b} m_{b} W_{a b}
$$

Alternatively, the continuity equation can be integrated, see Eq. (3). The evolution equation for the specific internal energy can be written as

$$
\frac{d u_{a}}{d t}=\sum_{b} m_{b}\left(\frac{P_{a}}{\rho_{a}^{2}}+\frac{1}{2} \Pi_{a b}\right) \vec{v}_{a b} \cdot \nabla_{a} W_{a b}
$$

and the momentum equation as

$$
\frac{d \vec{v}_{a}}{d t}=-\sum_{b} m_{b}\left(\frac{P_{a}}{\rho_{a}^{2}}+\frac{P_{b}}{\rho_{b}^{2}}+\Pi_{a b}\right) \nabla_{a} W_{a b} .
$$

Particles are advanced in time according to this equation. $\Pi_{a b}$ is the "standard" artificial viscosity term given in Eq. (59), more modern prescriptions are given in Eq. (67) and Eq. (68). Alternative forms of energy may be integrated forward in time, see, for example, Eq. (34).

\section{$3 \mathrm{SPH}$ from a variational principle}

In the previous section we had seen how the "vanilla ice" SPH equations can be derived directly from the Lagrangian form of the inviscid hydrodynamic equations. Although this form ensures conservation of energy, linear and angular momentum and works well in practice, the corresponding symmetries were enforced somewhat ad hoc. SPH can also be derived using nothing more than a suitable fluid Lagrangian, the first law of thermodynamics and a prescription on how to obtain a density estimate via summation $[165,201,146]$. 


\subsection{The Lagrangian and the Euler-Lagrange equations}

The Lagrangian of a perfect fluid is given by [202]

$$
L=\int \rho\left(\frac{v^{2}}{2}-u(\rho, s)\right) d V
$$

where $\rho$ is the mass density, $v$ the fluid velocity, $u$ the specific energy and $s$ the specific entropy. In SPH-discretization it reads

$$
L_{\mathrm{SPH}, \mathrm{h}}=\sum_{b} m_{b}\left(\frac{v_{b}^{2}}{2}-u\left(\rho_{b}, s_{b}\right)\right) .
$$

The discretized equations for the fluid are then derived by applying the EulerLagrange equations

$$
\frac{d}{d t}\left(\frac{\partial L}{\partial \vec{v}_{a}}\right)-\frac{\partial L}{\partial \vec{r}_{a}}=0
$$

where $\vec{r}_{a}$ and $\vec{v}_{a}$ refer to the position and velocity of particle $a$. The term in brackets yields the canonical particle momentum (like usual, we keep the particle mass fixed in time)

$$
\frac{\partial L}{\partial \vec{v}_{a}}=\frac{\partial}{\partial \vec{v}_{a}}\left[\sum_{b} m_{b}\left(\frac{v_{b}^{2}}{2}-u\left(\rho_{b}, s_{b}\right)\right)\right]=m_{a} \vec{v}_{a}
$$

and therefore, the first term in Eq. (105) yields the change of particle momentum $m_{a} \frac{d \vec{v}_{a}}{d t}$.

The second term in the Lagrangian acts like a potential, and if self-gravity is considered, $-\sum_{b} m_{b} u_{b}$ has to be replaced by $-\sum_{b} m_{b}\left(u_{b}+\Phi_{b}\right)$, where $\Phi_{b}$ is the gravitational potential [203]. In the following, we will only consider $\Phi_{b}=0$. The second term in Eq. (105) becomes

$$
\frac{\partial L}{\partial \vec{r}_{a}}=\frac{\partial}{\partial \vec{r}_{a}}\left[\sum_{b} m_{b}\left(\frac{v_{b}^{2}}{2}-u\left(\rho_{b}, s_{b}\right)\right)\right]=-\left.\sum_{b} m_{b} \frac{\partial u_{b}}{\partial \rho_{b}}\right|_{s} \cdot \frac{\partial \rho_{b}}{\partial \vec{r}_{a}} .
$$

We can make use of the first law of thermodynamics, Eq. (7), to find

$$
m_{a} \frac{d \vec{v}_{a}}{d t}=-\sum_{b} m_{b} \frac{P_{b}}{\rho_{b}^{2}} \frac{\partial \rho_{b}}{\partial \vec{r}_{a}} .
$$




\subsection{The density, its derivatives and "grad-h"-terms}

So far, we had ignored all extra terms that result from variable smoothing lengths $[204,146,147]$, this is what we address now. From now on, we use the smoothing length of the particle itself in the density summation,

$$
\rho_{a}=\sum_{b} m_{b} W\left(r_{a b}, h_{a}\right)
$$

and use Eq. (51) to adapt the smoothing length. Thus, $\rho_{a}$ depends on $h_{a}$ and vice versa, which requires an iteration to reach consistency.

If we take the changes of $h$ into account, the Lagrangian time derivative of the density is given by

$$
\begin{aligned}
\frac{d \rho_{a}}{d t} & =\frac{d}{d t}\left(\sum_{b} m_{b} W_{a b}\left(h_{a}\right)\right)=\sum_{b} m_{b}\left\{\frac{\partial W_{a b}\left(h_{a}\right)}{\partial r_{a b}} \frac{d r_{a b}}{d t}+\frac{\partial W_{a b}\left(h_{a}\right)}{\partial h_{a}} \frac{d h_{a}}{d t}\right\} \\
& =\sum_{b} m_{b} \frac{\partial W_{a b}\left(h_{a}\right)}{\partial r_{a b}} \hat{e}_{a b} \cdot \vec{v}_{a b}+\sum_{b} m_{b} \frac{\partial W_{a b}\left(h_{a}\right)}{\partial h_{a}} \cdot \frac{\partial h_{a}}{\partial \rho_{a}} \frac{d \rho_{a}}{d t} \\
& =\sum_{b} m_{b} \vec{v}_{a b} \cdot \nabla_{a} W_{a b}\left(h_{a}\right)+\frac{\partial h_{a}}{\partial \rho_{a}} \frac{d \rho_{a}}{d t} \sum_{b} m_{b} \frac{\partial W_{a b}\left(h_{a}\right)}{\partial h_{a}}
\end{aligned}
$$

where we have used Eqs. (24) and (26). If we collect the $d \rho_{a} / d t$-terms and introduce

$$
\Omega_{a} \equiv\left(1-\frac{\partial h_{a}}{\partial \rho_{a}} \sum_{b} m_{b} \frac{\partial W_{a b}\left(h_{a}\right)}{\partial h_{a}}\right)
$$

the time derivative of the density reads

$$
\frac{d \rho_{a}}{d t}=\frac{1}{\Omega_{a}} \sum_{b} m_{b} \vec{v}_{a b} \cdot \nabla_{a} W_{a b}\left(h_{a}\right)
$$

This is the generalization of the SPH expression for the density change that follows from Eq. (31). In a similar way, the spatial derivatives can be calculated

$$
\begin{aligned}
\frac{\partial \rho_{b}}{\partial \vec{r}_{a}} & =\sum_{k} m_{k}\left\{\nabla_{a} W_{b k}\left(h_{b}\right)+\frac{\partial W_{b k}\left(h_{b}\right)}{\partial h_{b}} \frac{\partial h_{b}}{\partial \rho_{b}} \frac{\partial \rho_{b}}{\partial \vec{r}_{a}}\right\} \\
& =\frac{1}{\Omega_{b}} \sum_{k} m_{k} \nabla_{a} W_{b k}\left(h_{b}\right) .
\end{aligned}
$$


To summarize: if derivatives of the smoothing length $h$ are accounted for, the "standard" SPH expressions for the density derivatives have to be corrected by factors $1 / \Omega$, see Eq. (111), (112) and (113).

\subsection{The SPH equations with "grad-h"-terms}

We keep again the masses fixed in time, so there is no need to solve the continuity equation. By inserting Eq. (112) into Eq. (30) we find the energy equation

$$
\frac{d u_{a}}{d t}=\frac{1}{\Omega_{a}} \frac{P_{a}}{\rho_{a}^{2}} \sum_{b} m_{b} \vec{v}_{a b} \cdot \nabla_{a} W_{a b}\left(h_{a}\right)
$$

For the momentum equation we need to insert the density gradient, Eq. (113), into Eq. (108)

$$
m_{a} \frac{d \vec{v}_{a}}{d t}=-\sum_{b} m_{b} \frac{P_{b}}{\rho_{b}^{2}} \nabla_{a} \rho_{b}=-\sum_{b} m_{b} \frac{P_{b}}{\rho_{b}^{2}}\left(\frac{1}{\Omega_{b}} \sum_{k} m_{k} \nabla_{a} W_{b k}\left(h_{b}\right)\right) .
$$

Using Eq. (25), the above equation becomes

$$
\begin{aligned}
m_{a} \frac{d \vec{v}_{a}}{d t} & =-\sum_{b} m_{b} \frac{P_{b}}{\rho_{b}^{2}} \frac{1}{\Omega_{b}} \sum_{k} m_{k} \nabla_{b} W_{k b}\left(h_{b}\right)\left(\delta_{b a}-\delta_{k a}\right) \\
& =-m_{a} \frac{P_{a}}{\rho_{a}^{2}} \frac{1}{\Omega_{a}} \sum_{k} m_{k} \nabla_{a} W_{k a}\left(h_{a}\right)+\sum_{b} m_{b} \frac{P_{b}}{\rho_{b}^{2}} \frac{1}{\Omega_{b}} m_{a} \nabla_{b} W_{a b}\left(h_{b}\right) \\
& =-m_{a} \frac{P_{a}}{\rho_{a}^{2}} \frac{1}{\Omega_{a}} \sum_{b} m_{b} \nabla_{a} W_{b a}\left(h_{a}\right)-m_{a} \sum_{b} m_{b} \frac{P_{b}}{\rho_{b}^{2}} \frac{1}{\Omega_{b}} \nabla_{a} W_{a b}\left(h_{b}\right) \\
& =-m_{a} \sum_{b} m_{b}\left(\frac{P_{a}}{\Omega_{a} \rho_{a}^{2}} \nabla_{a} W_{a b}\left(h_{a}\right)+\frac{P_{b}}{\Omega_{b} \rho_{b}^{2}} \nabla_{a} W_{a b}\left(h_{b}\right)\right) .
\end{aligned}
$$

Thus the final momentum equation reads

$$
\frac{d \vec{v}_{a}}{d t}=-\sum_{b} m_{b}\left(\frac{P_{a}}{\Omega_{a} \rho_{a}^{2}} \nabla_{a} W_{a b}\left(h_{a}\right)+\frac{P_{b}}{\Omega_{b} \rho_{b}^{2}} \nabla_{a} W_{a b}\left(h_{b}\right)\right) .
$$

Note that now all arbitrariness (such as the particular form of Eq. (28)) has been eliminated from the derivation. The "grad-h" terms increase the accuracy of SPH and the conservation properties in the presence of varying smoothing lengths. How important they are in practice depends on both the problem and the numerical resolution $[146,112]$. 


\section{SPH equations from a variational principle}

With

$$
\rho_{a}=\sum_{b} m_{b} W\left(r_{a b}, h_{a}\right) \quad \text { and } \quad h_{a}=\eta\left(\frac{m_{a}}{\rho_{a}}\right)^{1 / 3}
$$

the energy equation reads

$$
\frac{d u_{a}}{d t}=\frac{1}{\Omega_{a}} \frac{P_{a}}{\rho_{a}^{2}} \sum_{b} m_{b} \vec{v}_{a b} \cdot \nabla_{a} W_{a b}\left(h_{a}\right)
$$

and the momentum equation is

$$
\frac{d \vec{v}_{a}}{d t}=-\sum_{b} m_{b}\left(\frac{P_{a}}{\Omega_{a} \rho_{a}^{2}} \nabla_{a} W_{a b}\left(h_{a}\right)+\frac{P_{b}}{\Omega_{b} \rho_{b}^{2}} \nabla_{a} W_{a b}\left(h_{b}\right)\right),
$$

where

$$
\Omega_{a} \equiv\left(1-\frac{\partial h_{a}}{\partial \rho_{a}} \sum_{b} m_{b} \frac{\partial}{\partial h_{a}} W_{a b}\left(h_{a}\right)\right)
$$

are the so-called "grad-h-terms". Additional artificial dissipation terms as discussed in Sec. 2 need to be applied in order to resolve shocks.

\section{Relativistic SPH}

Several relativistic versions of SPH exist. Early formulations due to Mann $[205,206]$ were able to capture the overall solutions, but they were -by today's standards- not particularly accurate.

Laguna et al. [207] developed a 3D, general-relativistic SPH code. Due to their choice of variables their continuity equation contains a gravitational source term. This term requires to introduce SPH kernel functions for curved space-times which are in general not isotropic and invariant under translations. Moreover, they did not use a conservative form of the equations and their approach requires additional time derivatives of Lorentz factors which they approximated by first order finite differences. Similar to the approach of Mann, only mildly relativistic shocks could be treated. The approach of [207] is also the basis of the more recent code described in [208].

Kheyfets et al. [209] suggested an alternative approach in which they define the spatial kernel function in a local rest frame and assume that space is approximately flat in this frame. The interaction between particles requires the 
choice of a particular frame whose calculation requires the solution of four coupled algebraic equations. While this approach is completely covariant, it requires a large computational effort.

Like in grid-based methods, a conservative formulation is crucial to handle strong shocks. As we had seen in Sec.2.7, concepts of Riemann solvers were used as a guide to improve SPH's artificial viscosity by treating the interaction between two particles as a local Riemann problem [183]. This approach replaces wave propagation speeds by an appropriately chosen signal velocity. With this approach (together with evolving the total energy rather than the thermal energy) Chow and Monaghan [210] obtained accurate results even for ultra-relativistic flows.

More recently, Siegler and Riffert [211] and Siegler [212] have suggested a set of equations for both the special- and general-relativistic (fixed background metric) case. It is based on a formulation of the Lagrangian hydrodynamics equations in conservative form. By a particular choice of dynamical variables they avoid many complications that have plagued earlier relativistic SPH formulations. In particular, by choosing the relativistic rest mass density for the $\mathrm{SPH}$ formalism the continuity equation has the same form as in non-relativistic $\mathrm{SPH}$, time derivatives of Lorentz factors do not appear and flat space kernels can be used. This comes at the price of inverting the dynamically evolved variables into the physical variables. Siegler [212] was able to accurately simulate some test cases with Lorentz factors up to $\gamma=1000$.

A more elegant approach is based on the use of the discretized Lagrangian of a perfect fluid [201]. Guided by the canonical momentum and energy, suitable numerical variables can be chosen which lead to evolution equations that are similar to the Newtonian ones. Due to the consistent derivation from a Lagrangian the conservation of mass, energy, linear and angular momentum are guaranteed. This approach can be applied both to the special- and the general-relativistic case.

In recent years, SPH has also been applied to study flows in time-varying space-times. The first approaches used Post-Newtonian approximations [213216], more recently the conformal flatness approximation [217,218] has been implemented [219-221].

In the following subsections we derive the special- and general-relativistic SPH equations from a variational principle similar to [201].

\subsection{Special-relativistic SPH}

Here, we generalize the approach of Monaghan and Price [201] to include the special-relativistic "grad-h" terms. We use the conventions that the metric tensor has the signature $(-,+,+,+)$, Latin indices run from 1 to 3 , Greek ones from 0 to 3 with the zero component being time. In addition, the usual Einstein sum convention and $c=G=1$ are used and the flat spacetime metric is 
denoted by $\eta_{\mu \nu}$.

With these conventions, the line element reads

$$
d s^{2}=\eta_{\mu \nu} d x^{\mu} d x^{\nu}
$$

and the proper time is given by $d \tau^{2}=-d s^{2}$. The 4 -velocity is defined as

$$
U^{\mu}=\frac{d x^{\mu}}{d \tau}
$$

and, due to the above two relations, it is normalized to -1 :

$$
U_{\mu} U^{\mu}=\eta_{\mu \nu} \frac{d x^{\nu}}{d \tau} \frac{d x^{\mu}}{d \tau}=\frac{d s^{2}}{d \tau^{2}}=-1
$$

The velocity components can be written as

$$
\left(U^{\mu}\right)=(\gamma, \gamma \vec{v})
$$

where $\gamma$ is the Lorentz factor

$$
\gamma=\frac{1}{\sqrt{1-v^{2}}}
$$

with $\vec{v}$ being the 3-velocity. Due to Eq. (125) the 3-velocity can be expressed as

$$
v^{i}=\frac{U^{i}}{U^{0}}
$$

The 4-momentum of a particle with rest mass $m$ is given by

$$
\left(p^{\mu}\right)=\left(m U^{\mu}\right)=(\gamma m, \gamma m \vec{v})=(E, \vec{p}),
$$

where $E$ is the particle energy and $\vec{p}$ its relativistic momentum. The last expression is also correct for particles with vanishing rest mass.

\subsubsection{The Lagrangian}

Consistent with our overall strategy, we derive the SPH equations from a Lagrangian to ensure exact conservation. We start from the Lagrangian of a perfect fluid [222]

$$
L_{\mathrm{pf}, \mathrm{sr}}=-\int T^{\mu \nu} U_{\mu} U_{\nu} d V
$$




\section{The energy momentum tensor of a perfect fluid}

The energy-momentum tensor, $T^{\mu \nu}$, is a key element of relativistic hydrodynamics as it contains the sources of the relativistic gravitational field. Its components $T^{\mu \nu}$ are defined as "flux of $\mu$-component of the 4-momentum in $\nu$ direction". Thus, the $T^{00}$-component is the energy density, the $T^{0 j}$ component is the energy flux, the $T^{i 0}$ component is the momentum density and the $T^{i j}$ is the momentum flux. For a perfect fluid with zero viscosity and heat conduction it is given by

$$
T^{\mu \nu}=(P+e) U^{\mu} U^{\nu}+P \eta^{\mu \nu}
$$

where $P$ is the fluid pressure, $U^{\mu}$ the 4 -velocity and $e$ the energy density in the local rest frame. The conservation of energy and momentum can be expressed as

$$
T^{\mu \nu}, \nu=0
$$

where the comma denotes the partial derivative (in general relativity it has to be replaced by the covariant derivative, usually denoted by a semicolon). $\mu=0$ expresses energy and $\mu=i$ momentum conservation.

In the Newtonian limit (with $c=1$ ) the following relations hold $U^{0}=\gamma \simeq 1$, $U^{i} \simeq v^{i}, e \simeq \rho$ and $P / e \ll 1$. Thus the components read

$$
\begin{aligned}
& T^{00}=(P+e) U^{0} U^{0}+P \approx \rho \\
& T^{0 j}=(P+e) U^{0} U^{j} \approx \rho v^{j} \\
& T^{i j}=(P+e) U^{i} U^{j}+P \delta^{i j} \approx \rho v^{i} v^{j}+P \delta^{i j} .
\end{aligned}
$$

Therefore, $T^{0 \mu}{ }_{, \mu}=0$ reduces to the usual continuity equation

$$
T_{, \mu}^{0 \mu}=T_{, 0}^{00}+T^{0 j}{ }_{j}=\frac{\partial \rho}{\partial t}+\frac{\partial\left(\rho v^{j}\right)}{\partial x^{j}}=0
$$

and $T^{i \mu}{ }_{\mu}=0$ becomes

$$
T_{, \mu}^{i \mu}=T_{, 0}^{i 0}+T^{i j}{ }_{j}=\frac{\partial\left(\rho v^{i}\right)}{\partial t}+\frac{\partial\left(\rho v^{i} v^{j}\right)}{\partial x^{j}}+\frac{\partial P}{\partial x^{j}}=0
$$

which is the Euler equation.

This Lagrangian is, apart from the flat space volume element, the same as the general-relativistic one, see Sec. 4.2. The energy density, $e^{9}$, that is needed in

$\overline{9}$ This quantity is usually denoted by $\rho$ in the literature. To avoid confusion with the non-relativistic mass density that we have used previously, we choose a different 
the energy-momentum tensor, see Eq. (130), has contributions from the rest mass and the thermal energy:

$$
e=e_{\text {rest }}+e_{\text {therm }}=\rho_{\text {rest }} c^{2}+u \rho_{\text {rest }}=n m_{0} c^{2}\left(1+u / c^{2}\right),
$$

where $m_{0}$ is the baryon mass ${ }^{10}$ and $u$ is again the specific thermal energy and $n$ is the baryon number density, the latter two are measured in the local rest frame. Here, we have kept the $c^{2}$ s for clarity. From now on we will measure all our energies in units of $m_{0} c^{2}$ (and use $c \equiv 1$ ). With these conventions the energy momentum tensor reads

$$
T^{\mu \nu}=(n[1+u(n, s)]+P) U^{\mu} U^{\nu}+P \eta^{\mu \nu} .
$$

Here $s$ is the specific entropy per baryon in the local fluid rest frame and $P$ the fluid pressure. For a practical computation we fix a particular frame ("computing frame") and transform the quantities into this frame where necessary. Using Eq. (124), we can considerably simplify the above Lagrangian. The quantity under the integral becomes

$$
T^{\mu \nu} U_{\mu} U_{\nu}=\left\{(n[1+u]+P) U^{\mu} U^{\nu}\right\} U_{\mu} U_{\nu}+P \eta^{\mu \nu} U_{\mu} U_{\nu}=n(1+u)
$$

and the Lagrangian simplifies to

$$
L_{\mathrm{pf}, \mathrm{sr}}=-\int n(1+u) d V .
$$

The baryon number density in the rest frame of a fluid element, $n$, is related to the number density in our computing frame, $N$, by a Lorentz factor, $\gamma$. To see this, assume a volume in the local rest frame of a fluid element, $V_{\text {lrf }}$, that contains $k$ baryons. In general, this fluid element will move with some velocity with respect to our computing frame. If we assume that the motion is in, say, $x$-direction, the $x$-component of the volume element in the computing frame, $V_{\text {cf }}$, appears Lorentz-contracted by a factor $\gamma: V_{\text {cf }}=V_{\text {lrf }} / \gamma$. Since this volume element contains the same number of baryons, $k$, the density in the computing frame is

$$
N=\frac{k}{V_{\mathrm{cf}}}=\frac{k \gamma}{V_{\mathrm{lrf}}}=\gamma n
$$

As before, see Eq. (104), we want to discretize the Lagrangian to derive the $\mathrm{SPH}$ equations. We subdivide space in volumes $\Delta V_{b}$, so that a volume labeled

symbol here.

${ }^{10}$ Note that this quantity depends on the relative number of neutrons and protons, i.e. on the nuclear composition. 
by index $b$ contains $\nu_{b}=\Delta V_{b} N_{b}$ baryons, where $N_{b}$ is the baryon density at particle $b$ (all quantities measured in the computing frame). Or, the other way around, if a fluid parcel labeled by $b$ contains $\nu_{b}$ baryons, it has a volume

$$
\Delta V_{b}=\frac{\nu_{b}}{N_{b}}
$$

Similar to Eq.(12), we can use this volume element in the SPH-approximation of a quantity $f^{11}$ :

$$
f(\vec{r})=\sum_{b} f_{b} \frac{\nu_{b}}{N_{b}} W\left(\left|\vec{r}-\vec{r}_{b}\right|, h\right) .
$$

With this prescription the baryon number density in the computing frame at position of particle $a$ reads

$$
N_{a}=N\left(\vec{r}_{a}\right)=\sum_{b} \nu_{b} W\left(\left|\vec{r}_{a}-\vec{r}_{b}\right|, h_{a}\right)
$$

where we have used $h_{a}$ to conform with the non-relativistic version, Eq. (109). The latter can be recovered by the following replacements: $m_{b} \rightarrow \nu_{b}$ and $\rho_{b} \rightarrow N_{b}$. If we keep the baryon number per SPH particle, $\nu_{b}$, fixed, the total baryon number is conserved and there is no need to evolve a continuity equation. Analogously to Eq. (31) one could also discretize the continuity equation and calculate the density by integration. We adapt the smoothing length similar to the Newtonian case, see Eq. (51),

$$
h_{a}=\eta\left(\frac{\nu_{a}}{N_{a}}\right)^{1 / 3}
$$

which, again, requires an iteration for consistent values of $N_{a}$ and $h_{a}$. We can now discretize the Lagrangian of Eq. (140)

$$
L_{\mathrm{SPH}, \mathrm{sr}}=-\sum_{b} \frac{\nu_{b}}{N_{b}} n_{b}\left[1+u\left(n_{b}, s_{b}\right)\right]
$$

or, by using Eq. (141),

$$
L_{\mathrm{SPH}, \mathrm{sr}}=-\sum_{b} \frac{\nu_{b}}{\gamma_{b}}\left[1+u\left(n_{b}, s_{b}\right)\right]=-\sum_{b} \nu_{b} \sqrt{1-v_{b}^{2}}\left[1+u_{b}\right] .
$$

\footnotetext{
${ }^{11}$ For ease of notation we have again dropped the distinction between the function to be interpolated and the interpolant.
} 


\subsubsection{The momentum equation}

The Euler-Lagrange equations, Eq. (105), determine the evolution of specialrelativistic momentum:

$$
\vec{p}_{a} \equiv \frac{\partial L_{\mathrm{SPH}, \mathrm{sr}}}{\partial \vec{v}_{a}}=-\sum_{b} \nu_{b} \frac{\partial}{\partial \vec{v}_{a}}\left(\frac{1+u\left(n_{b}, s_{b}\right)}{\gamma_{b}}\right) .
$$

The specific energy $u$ depends, via the density, on the velocity:

$$
\frac{\partial u_{b}}{\partial \vec{v}_{a}}=\left(\frac{\partial u_{b}}{\partial n_{b}}\right)_{s} \frac{\partial n_{b}}{\partial \vec{v}_{a}}=\frac{P_{b}}{n_{b}^{2}} \frac{\partial n_{b}}{\partial \vec{v}_{a}},
$$

where we have used Eq. (8). Via Eq. (141) we have

$$
\frac{\partial n_{b}}{\partial \vec{v}_{a}}=N_{b} \frac{\partial}{\partial \vec{v}_{a}}\left(\frac{1}{\gamma_{b}}\right)=N_{b} \frac{\partial}{\partial \vec{v}_{a}}\left(1-v_{b}^{2}\right)^{1 / 2}=-N_{b} \gamma_{b} \vec{v}_{b} \delta_{a b} .
$$

Thus, the canonical momentum is

$$
\begin{aligned}
\vec{p}_{a} & =\frac{\partial L_{\mathrm{SPH}, \mathrm{sr}}}{\partial \vec{v}_{a}}=-\sum_{b} \nu_{b}\left[1+u_{b}\right] \frac{\partial}{\partial \vec{v}_{a}}\left(\frac{1}{\gamma_{b}}\right)-\sum_{b} \frac{\nu_{b}}{\gamma_{b}} \frac{\partial u_{b}}{\partial \vec{v}_{a}} \\
& =\sum_{b} \nu_{b}\left(\overrightarrow{v_{b}} \gamma_{b} \delta_{a b}\right)\left[1+u_{b}\right]+\sum_{b} \frac{\nu_{b}}{\gamma_{b}}\left(\frac{P_{b}}{n_{b}^{2}}\right)\left(N_{b} \gamma_{b} \vec{v}_{b} \delta_{a b}\right) \\
& =\nu_{a} \gamma_{a} \vec{v}_{a}\left(1+u_{a}+\frac{P_{a}}{n_{a}}\right),
\end{aligned}
$$

where we have again used Eq. (141), the term in brackets after the last equal sign is the enthalpy per baryon. Obviously, in relativity the pressure contributes to the momentum density. We define the canonical momentum per baryon as

$$
\vec{S}_{a} \equiv \gamma_{a} \vec{v}_{a}\left(1+u_{a}+\frac{P_{a}}{n_{a}}\right),
$$

whose time evolution is governed by $\partial L_{\mathrm{SPH}, \mathrm{sr}} / \partial \vec{r}_{a}$, see Eq. (105), and which requires the gradient of the number density. The latter is obtained similar to the Newtonian case, see Eq. (113),

$$
\frac{\partial N_{b}}{\partial \vec{r}_{a}}=\sum_{k} \nu_{k}\left\{\frac{\partial W\left(r_{b k}, h_{b}\right)}{\partial r_{b k}} \frac{\partial r_{b k}}{\partial \vec{r}_{a}}+\frac{\partial W\left(r_{b k}, h_{b}\right)}{\partial h_{b}} \frac{\partial h_{b}}{\partial N_{b}} \frac{\partial N_{b}}{\partial \vec{r}_{a}}\right\}
$$




$$
=\frac{1}{\tilde{\Omega}_{b}} \sum_{k} \nu_{k} \nabla_{a} W_{b k}\left(h_{b}\right)\left(\delta_{b a}-\delta_{k a}\right)
$$

with

$$
\tilde{\Omega}_{b}=1-\frac{\partial h_{b}}{\partial N_{b}} \sum_{k} \nu_{k} \frac{\partial W\left(r_{b k}, h_{b}\right)}{\partial h_{b}} .
$$

Thus

$$
\begin{aligned}
\frac{\partial L_{\mathrm{SPH}, \mathrm{sr}}}{\partial \vec{r}_{a}} & =-\sum_{b} \frac{\nu_{b}}{\gamma_{b}} \frac{\partial u_{b}}{\partial \vec{r}_{a}}=-\sum_{b} \frac{\nu_{b}}{\gamma_{b}} \frac{P_{b}}{n_{b}^{2}} \frac{\partial n_{b}}{\partial \vec{r}_{a}}=-\sum_{b} \frac{\nu_{b}}{\gamma_{b}^{2}} \frac{P_{b}}{n_{b}^{2}} \frac{\partial N_{b}}{\partial \vec{r}_{a}} \\
& =-\sum_{b, k} \frac{\nu_{b} \nu_{k}}{\gamma_{b}^{2}} \frac{P_{b}}{\tilde{\Omega}_{b} n_{b}^{2}} \nabla_{a} W_{b k}\left(h_{b}\right)\left(\delta_{b a}-\delta_{k a}\right) \\
& =-\nu_{a} \sum_{b} \nu_{b}\left\{\frac{P_{a}}{\tilde{\Omega}_{a} N_{a}^{2}} \nabla_{a} W_{a b}\left(h_{a}\right)+\frac{P_{b}}{\tilde{\Omega}_{b} N_{b}^{2}} \nabla_{a} W_{a b}\left(h_{b}\right)\right\},
\end{aligned}
$$

where we have used Eqs. (8), (141), (144), (153), and (26). With Eq. (155) and Eq. (151), the special-relativistic SPH momentum equation becomes

$$
\frac{d \vec{S}_{a}}{d t}=-\sum_{b} \nu_{b}\left\{\frac{P_{a}}{\tilde{\Omega}_{a} N_{a}^{2}} \nabla_{a} W_{a b}\left(h_{a}\right)+\frac{P_{b}}{\tilde{\Omega}_{b} N_{b}^{2}} \nabla_{a} W_{a b}\left(h_{b}\right)\right\} .
$$

This form can be obtained from the non-relativistic case, Eq. (117), by the following replacements: $\vec{v}_{a} \rightarrow \vec{S}_{a}, m_{k} \rightarrow \nu_{k}, \Omega_{k} \rightarrow \tilde{\Omega}_{k}$ and $\rho_{k} \rightarrow N_{k}$.

Since the Lagrangian is invariant under infinitesimal translation the total canonical momentum

$$
\vec{P}=\sum_{b} \vec{p}_{b}=\sum_{b} \nu_{b} \vec{S}_{b}=\sum_{b} \nu_{b} \gamma_{b} \vec{v}_{b}\left(1+u_{b}+\frac{P_{b}}{n_{b}}\right)
$$

is conserved. Similarly, due to invariance under rotation, the angular momentum

$$
\vec{L}=\sum_{b} \vec{r}_{b} \times \vec{p}_{b}=\sum_{b} \nu_{b} \vec{r}_{b} \times \vec{S}_{b}=\sum_{b} \nu_{b} \gamma_{b}\left(1+u_{b}+\frac{P_{b}}{n_{b}}\right) \vec{r}_{b} \times \vec{v}_{b}
$$

is conserved by construction. 


\subsubsection{The energy equation}

To choose a suitable numerical energy variable we start from the conserved canonical energy (no explicit time dependence in the Lagrangian)

$$
E \equiv \sum_{a} \frac{\partial L_{\mathrm{SPH}, \mathrm{sr}}}{\partial \vec{v}_{a}} \cdot \vec{v}_{a}-L_{\mathrm{SPH}, \mathrm{sr}}
$$

With Eqs. (147) and Eq. (151) the energy reads

$$
E=\sum_{a} \nu_{a}\left(\vec{v}_{a} \cdot \vec{S}_{a}+\frac{1+u_{a}}{\gamma_{a}}\right)=\sum_{a} \nu_{a} \hat{\epsilon}_{a}
$$

where, we have defined

$$
\hat{\epsilon}_{a} \equiv \vec{v}_{a} \cdot \vec{S}_{a}+\frac{1+u_{a}}{\gamma_{a}} .
$$

For later use, we note that by using $v_{a}^{2}=1-1 / \gamma_{a}^{2}$ and Eq. (141) we can express $\hat{\epsilon}_{a}$ as

$$
\hat{\epsilon}_{a}=\gamma_{a} v_{a}^{2}\left(1+u_{a}+\frac{P_{a}}{n_{a}}\right)+\frac{1+u_{a}}{\gamma_{a}}=\gamma_{a}\left(1+u_{a}+\frac{P_{a}}{n_{a}}\right)-\frac{P_{a}}{N_{a}} .
$$

To find the evolution equation for $\hat{\epsilon}_{a}$, we need the time derivative of the second term in Eq. (161). Ideally, it should not contain a time derivative of the Lorentz factor since such terms are known to destabilize numerical schemes [223]. Using

$$
\frac{d \gamma_{a}}{d t}=\gamma_{a}^{3} \vec{v}_{a} \cdot \frac{d \vec{v}_{a}}{d t} \quad \text { and } \quad \frac{d n_{a}}{d t}=\frac{d}{d t}\left(\frac{N_{a}}{\gamma_{a}}\right)=\frac{1}{\gamma_{a}} \frac{d N_{a}}{d t}-N_{a} \gamma_{a} \vec{v}_{a} \cdot \frac{d \vec{v}_{a}}{d t}
$$

we find

$$
\begin{aligned}
\frac{d}{d t}\left(\frac{1+u_{a}}{\gamma_{a}}\right) & =\frac{1}{\gamma_{a}^{2}}\left(\gamma_{a} \frac{d u_{a}}{d t}-\left(1+u_{a}\right) \frac{d \gamma_{a}}{d t}\right) \\
& =\frac{1}{\gamma_{a}} \frac{P_{a}}{n_{a}^{2}} \frac{d n_{a}}{d t}-\frac{1+u_{a}}{\gamma_{a}^{2}} \gamma_{a}^{3} \vec{v}_{a} \cdot \frac{d \vec{v}_{a}}{d t} \\
& =\frac{P_{a}}{N_{a}^{2}} \frac{d N_{a}}{d t}-\left(1+u_{a}+\frac{P_{a}}{n_{a}}\right) \gamma_{a} \vec{v}_{a} \cdot \frac{d \vec{v}_{a}}{d t} \\
& =\frac{P_{a}}{N_{a}^{2}} \frac{d N_{a}}{d t}-\vec{S}_{a} \cdot \frac{d \vec{v}_{a}}{d t},
\end{aligned}
$$


where we used Eqs. (8), (141) and (163). With equation (164) at hand, we can take the derivative of Eq. (161)

$$
\begin{aligned}
\frac{d \hat{\epsilon}_{a}}{d t} & =\frac{d}{d t}\left\{\vec{v}_{a} \cdot \vec{S}_{a}+\frac{1+u_{a}}{\gamma_{a}}\right\} \\
& =\vec{S}_{a} \cdot \frac{d \vec{v}_{a}}{d t}+\vec{v}_{a} \cdot \frac{d \vec{S}_{a}}{d t}+\frac{P_{a}}{N_{a}^{2}} \frac{d N_{a}}{d t}-\vec{S}_{a} \cdot \frac{d \vec{v}_{a}}{d t} \\
& =\vec{v}_{a} \cdot \frac{d \vec{S}_{a}}{d t}+\frac{P_{a}}{N_{a}^{2}} \frac{d N_{a}}{d t}
\end{aligned}
$$

i.e. apart from the relativistic momentum equation (156), we need the Lagrangian time derivative of the number density in the computing frame:

$$
\begin{aligned}
\frac{d N_{a}}{d t} & =\sum_{b} \nu_{b}\left\{\frac{\partial W_{a b}\left(h_{a}\right)}{\partial r_{a b}} \frac{d r_{a b}}{d t}+\frac{\partial W_{a b}}{\partial h_{a}} \frac{\partial h_{a}}{\partial N_{a}} \frac{d N_{a}}{d t}\right\} \\
& =\frac{1}{\tilde{\Omega}_{a}} \sum_{b} \nu_{b} \vec{v}_{a b} \cdot \nabla_{a} W_{a b}\left(h_{a}\right),
\end{aligned}
$$

where we have used Eq. (154). If we insert Eqs. (156) and (166) into Eq. (165) the energy evolution equation becomes

$$
\frac{d \hat{\epsilon}_{a}}{d t}=-\sum_{b} \nu_{b}\left(\frac{P_{a} \vec{v}_{b}}{\tilde{\Omega}_{a} N_{a}^{2}} \cdot \nabla_{a} W_{a b}\left(h_{a}\right)+\frac{P_{b} \vec{v}_{a}}{\tilde{\Omega}_{b} N_{b}^{2}} \cdot \nabla_{a} W_{a b}\left(h_{b}\right)\right) .
$$

This relativistic energy equation looks similar to the non-relativistic equation for the thermokinetic energy, see Eq. (34).

\subsubsection{Recovery of the primitive variables}

Due to our choice of the numerical variables neither the momentum nor the energy equation contain time derivatives of Lorentz factors. Such terms restricted earlier versions of relativistic SPH to moderate Lorentz factors only. But this comes at the price that the physical variables need to be recovered at every time step from the numerical ones, a price that relativistic Eulerian codes also have to pay.

At the end of a time step $\gamma / v, u$ and $n$ need to be calculated from the updated numerical quantities $N, \vec{S}$ and $\hat{\epsilon}$. One can express all variables in the equation of state

$$
P_{a}=(\Gamma-1) n_{a} u_{a}
$$



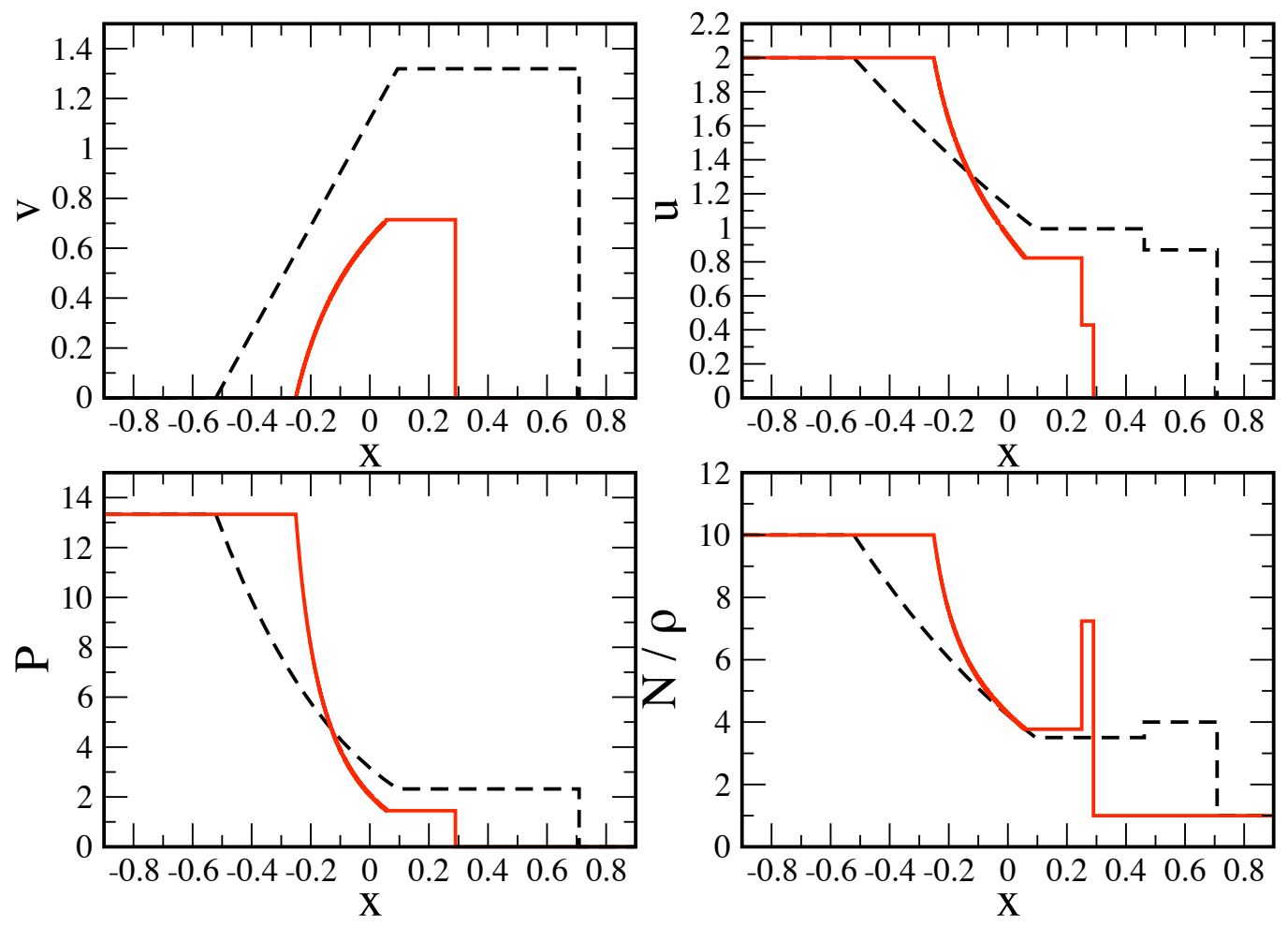

Fig. 8. Mildly relativistic $\left(\gamma_{\max } \approx 1.4\right)$ shock tube test: comparison between the Newtonian (dashed) and the special-relativistic results (solid, red). Upper left: velocity in units of $c$, upper right: thermal energy, lower left: pressure, lower right: (computing frame) number density $N$ and Newtonian mass density $\rho$.

as a function of the updated numerical variables and the pressure $P_{a}$. The resulting equation is solved numerically for $P_{a}$ and once it is found, the other physical variables can be recovered [224,183]. From Eqs. (152) and (162) one finds

$$
\vec{v}_{a}=\frac{\vec{S}_{a}}{\hat{\epsilon}_{a}+P_{a} / N_{a}}
$$

and thus the Lorentz factor is

$$
\gamma_{a}=\frac{1}{\sqrt{1-S_{a}^{2} /\left(\hat{\epsilon}_{a}+P_{a} / N_{a}\right)^{2}}}
$$


From Eq. (169) we have

$$
\left(\hat{\epsilon}_{a}+\frac{P_{a}}{\gamma_{a} n_{a}}\right) \vec{v}_{a}=\vec{S}_{a}=\gamma_{a} \vec{v}_{a}\left(1+u_{a}+\frac{P_{a}}{n_{a}}\right)
$$

which can be solved for

$$
u_{a}=\frac{\hat{\epsilon}_{a}}{\gamma_{a}}+\frac{P_{a}}{\gamma_{a} N_{a}}\left(1-\gamma_{a}^{2}\right)-1
$$

With aid of Eqs. (141) and (172), Eq. (168) can be solved, e.g. via a NewtonRaphson scheme, for the new pressure $P_{a}$. Once $P_{a}$ is known, the Lorentz factor can be calculated from Eq. (170), the specific energy from Eq. (172) and the velocity from Eq. (169).

\subsubsection{Numerical tests}

To explore the performance of this SPH formulation we show a numerical test that can be considered a special-relativistic generalization of Sod's shock tube test, see Sec. 2.7. This test has become a widespread benchmark for relativistic hydrodynamics codes, see for example [224,210,211,225,226]. The test is performed with a polytropic equation of state with exponent $\Gamma=5 / 3$, vanishing initial velocities everywhere, the left state has a pressure $P_{L}=40 / 3$ and a density $N_{L}=10$, while the right state is prepared with $P_{R}=10^{-6}$ and $N_{R}=1$. Although the resulting velocities are only mildly relativstic $\left(\gamma_{\max } \approx 1.4\right)$, the deviations from the purely Newtonian result are already substantial. This is demonstrated in Fig. 8, where we compare the exact solutions for these initial conditions for the Newtonian (dashed) and the special-relativistic case (solid). Fig. 9 shows the SPH result (black circles, about 3000 particles) at $t=0.35$ together with the exact solution (red, solid line). The general agreement is excellent, noticeable deviations are only observed in the form of a slightly smeared out contact discontinuity at $x \approx 0.25$. A striking difference to earlier SPH formulations $[207,211]$ is the absence of any spike in $u$ and $P$ at the contact discontinuity.

This SPH formulation has been further explored in a large set of specialrelativistic benchmark tests [227]. As expected, it performs very well in pure advection problems. Maybe more remarkable, it also shows convincing results in extremely strong, relativistic shocks. For example, it yields accurate solutions in a wall shock test with a Lorentz factor of $\gamma=50$ 000. The specialrelativistic "grad-h"-terms generally improve the accuracy, but (at least in the performed set of tests) only to a moderate extent. For more details we refer to $[227]$. 


\section{Summary of the special-relativistic SPH equations}

The momentum equation derived from the special-relativistic Lagrangian reads

$$
\frac{d \vec{S}_{a}}{d t}=-\sum_{b} \nu_{b}\left\{\frac{P_{a}}{\tilde{\Omega}_{a} N_{a}^{2}} \nabla_{a} W_{a b}\left(h_{a}\right)+\frac{P_{b}}{\tilde{\Omega}_{b} N_{b}^{2}} \nabla_{a} W_{a b}\left(h_{b}\right)\right\}
$$

where

$$
\vec{S}_{a}=\gamma_{a} \vec{v}_{a}\left(1+u_{a}+\frac{P_{a}}{n_{a}}\right)
$$

and

$$
\tilde{\Omega}_{b}=1-\frac{\partial h_{b}}{\partial N_{b}} \sum_{k} \nu_{k} \frac{\partial W\left(r_{b k}, h_{b}\right)}{\partial h_{b}} .
$$

The (canonical) energy variable

$$
\hat{\epsilon}_{a}=\vec{v}_{a} \cdot \vec{S}_{a}+\frac{1+u_{a}}{\gamma_{a}}
$$

is evolved according to

$$
\frac{d \hat{\epsilon}_{a}}{d t}=-\sum_{b} \nu_{b}\left(\frac{P_{a} \vec{v}_{b}}{\tilde{\Omega}_{a} N_{a}^{2}} \cdot \nabla_{a} W_{a b}\left(h_{a}\right)+\frac{P_{b} \vec{v}_{a}}{\tilde{\Omega}_{b} N_{b}^{2}} \cdot \nabla_{a} W_{a b}\left(h_{b}\right)\right) .
$$

The computing frame number density can be found either by summation,

$$
N_{a}=\sum_{b} \nu_{b} W_{a b}\left(h_{a}\right)
$$

or, alternatively, by integration of

$$
\frac{d N_{a}}{d t}=\frac{1}{\tilde{\Omega}_{a}} \sum_{b} \nu_{b} \vec{v}_{a b} \cdot \nabla_{a} W_{a b}\left(h_{a}\right)
$$

\subsection{General-relativistic SPH on a fixed background metric}

\subsubsection{The strategy}

We now generalize the previous approach by assuming that a fixed metric is given as a function of the coordinates and that corrections to the metric

induced by the fluid are negligible. Such a situation occurs, for example, during 

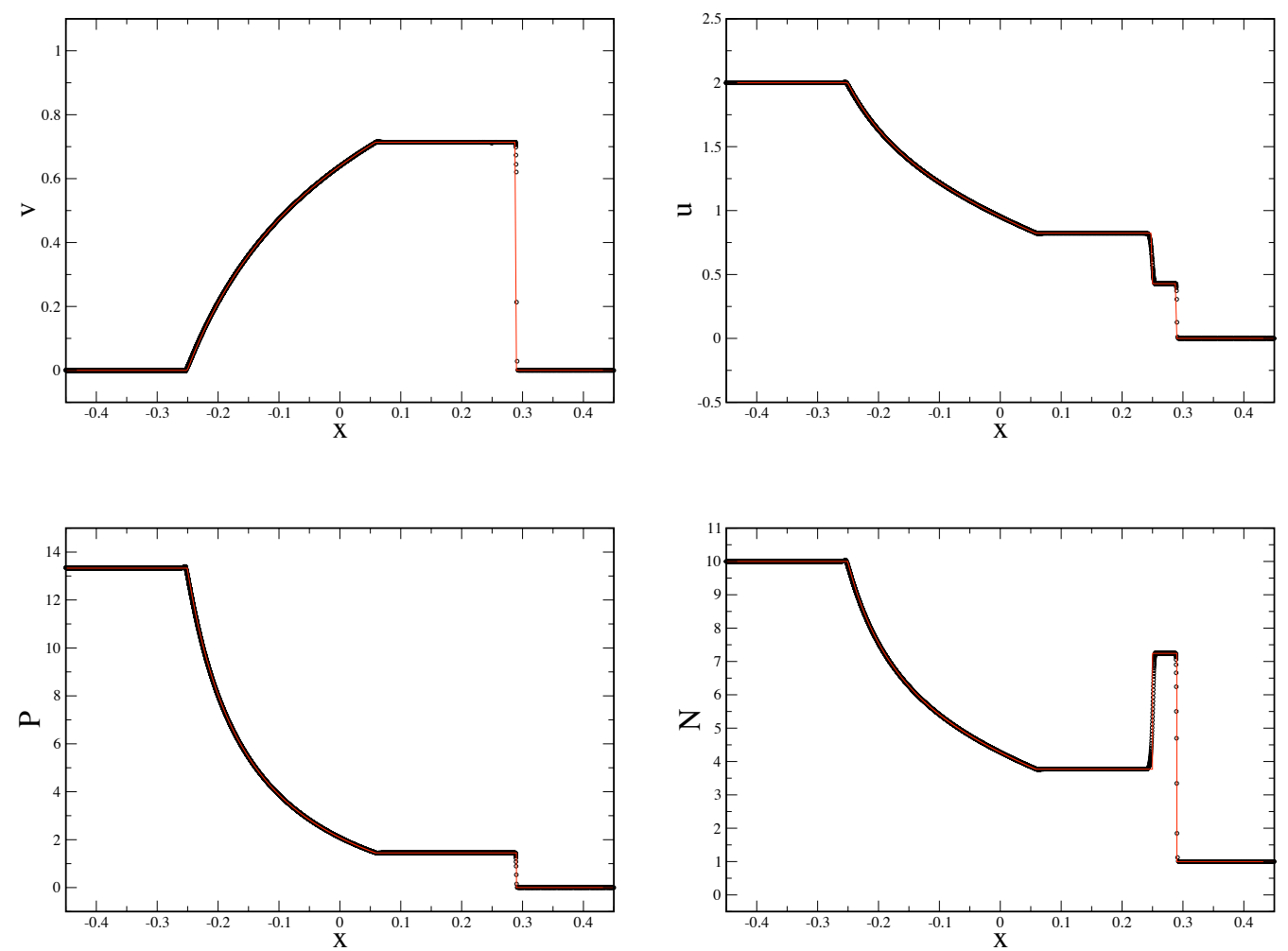

Fig. 9. SPH solution of the relativistic shock tube test of [224] (shown are 3000 particles; upper left: velocity in units of $c$, upper right: thermal energy, lower left: pressure, lower right: (computing frame) number density $N$ ). The black circles show the SPH solution, the red line marks the exact solution [226].

the tidal disruption of a star by a supermassive black hole in the center of a galaxy. Again, we start from the discretized Lagrangian of an ideal fluid and use the canonical momentum/energy as a guide for the choice of the numerical variables. As a matter of course, this new set of equations should reduce in the flat-space limit to the special-relativistic equations, see Eq. (173) - (178), which, in their low-velocity limit, should be equivalent to the non-relativistic, standard SPH equations.

\subsubsection{The Lagrangian}

We start from the general-relativistic Lagrangian of a perfect fluid [222]

$$
L_{\mathrm{pf}, \mathrm{GR}}=-\int T^{\mu \nu} U_{\mu} U_{\nu} \sqrt{-g} d V
$$

where $\sqrt{-g} d V$ is the proper volume element and $g=\operatorname{det}\left(g_{\mu \nu}\right)$ is the determinant of the metric tensor, $g_{\mu \nu}$. As before, the energy momentum tensor is 
defined by

$$
T^{\mu \nu}=\{n[1+u(n, s)]+P\} U^{\mu} U^{\nu}+P g^{\mu \nu},
$$

where the fluid quantities are measured in their local rest frame and the fourvelocity is defined as in Eq. (125). Obviously,

$$
\frac{d \tau}{d t}=\left(-g_{\mu \nu} \frac{d x^{\mu}}{d t} \frac{d x^{\nu}}{d t}\right)^{\frac{1}{2}}=\left(-g_{\mu \nu} v^{\mu} v^{\nu}\right)^{\frac{1}{2}}
$$

where we used $v^{\rho}=d x^{\rho} / d t$. Comparing with the special-relativistic relation $d \tau / d t=1 / \gamma$, one sees that the quantity

$$
\Theta \equiv\left(-g_{\mu \nu} v^{\mu} v^{\nu}\right)^{-\frac{1}{2}}
$$

takes over the role of and reduces in the flat spacetime limit to the specialrelativistic Lorentz factor $\gamma=1 / \sqrt{1-v^{2}}$. Similar to Eq. (127) we can also write

$$
v^{\mu}=\frac{d x^{\mu}}{d t}=\frac{d x^{\mu}}{d \tau} \frac{d \tau}{d t}=\frac{U^{\mu}}{U^{0}}
$$

where we have used $d t / d \tau=U^{0}=\Theta$, see Eq. (182). Using the normalization condition of the four-velocity, Eq. (124), the Lagrangian reduces to

$$
\begin{aligned}
L_{\mathrm{pf}, \mathrm{GR}} & =-\int\left[\{n(1+u)+P\} U^{\mu} U^{\nu} U_{\mu} U_{\nu}+P g^{\mu \nu} U_{\mu} U_{\nu}\right] \sqrt{-g} d V \\
& =-\int n[1+u(n, s)] \sqrt{-g} d V
\end{aligned}
$$

which is, of course, up to the volume element, the same result as in special relativity, Eq.(140).

To discretize the Lagrangian, we first have to find a suitable density variable so that we can apply the usual SPH discretization prescription. The generalrelativistic baryon number conservation equation reads

$$
\frac{1}{\sqrt{-g}} \frac{\partial}{\partial x^{\nu}}\left(\sqrt{-g} U^{\nu} n\right)=0
$$

see e.g. [228], and this suggests to use the modified number density

$$
N^{*}=\sqrt{-g} U^{0} n=\sqrt{-g} \Theta n,
$$


since it can be written with the help of Eq. (184) as

$$
\frac{\partial N^{*}}{\partial t}+\frac{\partial\left(N^{*} v^{i}\right)}{\partial x^{i}}=0
$$

Eq. (187) generalizes the earlier relation between the computing frame and the local rest frame, Eq. (141). We use $N^{*}$ in the SPH interpolations

$$
f(\vec{r})=\sum_{b} f_{b} \frac{\nu_{b}}{N_{b}^{*}} W\left(\left|\vec{r}-\vec{r}_{b}\right|\right)
$$

where

$$
\nu_{b}=N_{b}^{*} \Delta V_{b}=n_{b} \Theta_{b} \sqrt{-g_{b}} \Delta V_{b}
$$

is the number of baryons contained in the volume $\sqrt{-g_{b}} \Delta V_{b}$. Applying Eq. (189) to $N^{*}$ one obtains a density estimate by summation

$$
N_{a}^{*}=\sum_{b} \nu_{b} W_{a b}\left(h_{a}\right)
$$

similar to the earlier results, Eqs. (109) and (144). We can now use the relation between $N^{*}$ and $n$, Eq. (187), to write the Lagrangian as

$$
L_{\mathrm{pf}, \mathrm{GR}}=-\int \frac{N^{*}}{\sqrt{-g} \Theta}[1+u(n, s)] \sqrt{-g} d V=-\int \frac{N^{*}}{\Theta}[1+u(n, s)] d V,
$$

i.e. the metric tensor contribution $\sqrt{-g}$ drops out, and using $\Delta V_{b}=\nu_{b} / N_{b}^{*}$, the $\mathrm{SPH}$ form of the Lagrangian reads

$$
L_{\mathrm{SPH}, \mathrm{GR}}=-\sum_{b} \frac{\nu_{b}}{\Theta_{b}}\left[1+u\left(n_{b}, s_{b}\right)\right]
$$

In the flat spacetime limit $\Theta_{b} \rightarrow \gamma_{b}$ and we recover the discretized, specialrelativistic Lagrangian, Eq. (147).

\subsubsection{The momentum equation}

To identify a suitable numerical variable, we use like before, see Eq. (152), the canonical momentum

$$
\frac{\partial L}{\partial v_{a}^{i}}=-\sum_{b} \nu_{b}\left(\frac{\partial}{\partial v_{a}^{i}} \frac{1}{\Theta_{b}}\right)\left[1+u_{b}\right]-\sum_{b} \frac{\nu_{b}}{\Theta_{b}}\left(\frac{\partial}{\partial v_{a}^{i}} u_{b}\right)
$$


We thus need

$$
\frac{\partial}{\partial v_{a}^{i}}\left(\frac{1}{\Theta_{b}}\right)=\frac{\partial}{\partial v_{a}^{i}}\left(-g_{\mu \nu} v^{\mu} v^{\nu}\right)_{b}^{1 / 2}=-\Theta_{b}\left(g_{i \mu} v^{\mu}\right)_{a} \delta_{a b}
$$

and the derivative $\partial u_{b} / \partial v_{a}^{i}$, where, like in the special-relativistic case, the velocity dependence comes from the Lorentz-factor-like quantity $\Theta$ relating the densities in the local frame and the computing frame

$$
\begin{aligned}
\frac{\partial u_{b}}{\partial v_{a}^{i}} & =\frac{\partial u_{b}}{\partial n_{b}} \frac{\partial n_{b}}{\partial v_{a}^{i}}=\frac{P_{b}}{n_{b}^{2}} \frac{\partial}{\partial v_{a}^{i}}\left(\frac{N_{b}^{*}}{\sqrt{-g}_{b} \Theta_{b}}\right)=\frac{P_{b}}{n_{b}^{2}} \frac{N_{b}^{*}}{\sqrt{-g_{b}}} \frac{\partial}{\partial v_{a}^{i}}\left(\frac{1}{\Theta_{b}}\right) \\
& =-\frac{P_{b}}{n_{b}^{2}} \frac{N_{b}^{*}}{\sqrt{-g_{b}}} \Theta_{b}\left(g_{i \mu} v^{\mu}\right)_{a} \delta_{a b} .
\end{aligned}
$$

Here we have used Eqs. (8), (187) and (195). By inserting Eqs. (195) and (196) into Eq. (194) we find

$$
\begin{aligned}
\frac{\partial L}{\partial v_{a}^{i}} & =-\sum_{b} \nu_{b}\left[1+u_{b}\right]\left(-\Theta_{b}\left(g_{i \mu} v^{\mu}\right)_{a} \delta_{a b}\right)-\sum_{b} \frac{\nu_{b}}{\Theta_{b}}\left(-\frac{P_{b}}{n_{b}^{2}} \frac{N_{b}^{*}}{\sqrt{-g_{b}}} \Theta_{b}\left(g_{i \mu} v^{\mu}\right)_{a} \delta_{a b}\right) \\
& =\nu_{a} \Theta_{a}\left(1+u_{a}+\frac{P_{a}}{n_{a}^{2}} \frac{N_{a}^{*}}{\sqrt{-g_{a}} \Theta_{a}}\right)\left(g_{i \mu} v^{\mu}\right)_{a} \\
& =\nu_{a} \Theta_{a}\left(1+u_{a}+\frac{P_{a}}{n_{a}}\right)\left(g_{i \mu} v^{\mu}\right)_{a},
\end{aligned}
$$

where Eq. (187) has been used. This is the $i$-component of the canonical momentum and, like before, we use the canonical momentum per baryon,

$$
S_{i, a} \equiv \frac{1}{\nu_{a}} \frac{\partial L}{\partial v_{a}^{i}}=\Theta_{a}\left(1+u_{a}+\frac{P_{a}}{n_{a}}\right)\left(g_{i \mu} v^{\mu}\right)_{a}
$$

as numerical variable. Again, the term in the first bracket is the specific enthalpy. In the flat-space limit, $\Theta_{a} \rightarrow \gamma_{a}, g_{\mu \nu} \rightarrow \eta_{\mu \nu}$ and $g_{i \mu} v^{\mu} \rightarrow v_{i}=v^{i}$, this expression reduces exactly to the special-relativistic case, Eq. (174), as it should.

The evolution of $S_{i, a}$ is determined by the Euler-Lagrange equations, so we need

$$
\frac{\partial L}{\partial x_{a}^{i}}=-\sum_{b} \nu_{b}\left(\frac{\partial}{\partial x_{a}^{i}} \frac{1}{\Theta_{b}}\right)\left[1+u_{b}\right]-\sum_{b} \frac{\nu_{b}}{\Theta_{b}}\left(\frac{\partial u_{b}}{\partial x_{a}^{i}}\right) .
$$

The first derivative can be written as 


$$
\left(\frac{\partial}{\partial x_{a}^{i}} \frac{1}{\Theta_{b}}\right)=\frac{\partial}{\partial x_{a}^{i}}\left(-g_{\mu \nu} v^{\mu} v^{\nu}\right)_{b}^{\frac{1}{2}}=-\frac{\Theta_{b}}{2}\left(\frac{\partial g_{\mu \nu}}{\partial x_{a}^{i}}\right)_{b} v_{b}^{\mu} v_{b}^{\nu} \delta_{a b}
$$

and due to Eq. (184)

$$
v^{\mu} v^{\nu}=\frac{U^{\mu} U^{\nu}}{\Theta^{2}}
$$

so it becomes

$$
\left(\frac{\partial}{\partial x_{a}^{i}} \frac{1}{\Theta_{b}}\right)=-\left(\frac{U^{\mu} U^{\nu}}{2 \Theta}\right)_{b}\left(\frac{\partial g_{\mu \nu}}{\partial x_{a}^{i}}\right)_{b} \delta_{a b}
$$

The derivative of the internal energy is

$$
\begin{aligned}
\frac{\partial u_{b}}{\partial x_{a}^{i}}= & \frac{\partial u_{b}}{\partial n_{b}} \frac{\partial n_{b}}{\partial x_{a}^{i}}=\frac{P_{b}}{n_{b}^{2}} \frac{\partial}{\partial x_{a}^{i}}\left(\frac{N_{b}^{*}}{\sqrt{-g_{b}} \Theta_{b}}\right) \\
= & \frac{P_{b}}{n_{b}^{2} \sqrt{-g_{b}} \Theta_{b}}\left(\frac{\partial N_{b}^{*}}{\partial x_{a}^{i}}\right)+\frac{P_{b} N_{b}^{*}}{n_{b}^{2} \sqrt{-g}}\left(\frac{\partial}{\partial x_{a}^{i}} \frac{1}{\Theta_{b}}\right) \\
& +\frac{P_{b} N_{b}^{*}}{n_{b}^{2} \Theta_{b}}\left(\frac{\partial}{\partial x_{a}^{i}} \frac{1}{\sqrt{-g}}\right),
\end{aligned}
$$

where we have used the first law of thermodynamics, Eq. (8). By using Eq. (25), the derivative of the number density becomes

$$
\frac{\partial N_{b}^{*}}{\partial x_{a}^{i}}=\frac{\partial}{\partial x_{a}^{i}}\left(\sum_{k} \nu_{k} W_{b k}\right)=\sum_{k} \nu_{k} \frac{\partial W_{b k}}{\partial x_{b}^{i}}\left(\delta_{b a}-\delta_{k a}\right) .
$$

The last remaining derivative is

$$
\left(\frac{\partial}{\partial x_{a}^{i}} \frac{1}{\sqrt{-g}}\right)=-\left(\frac{g^{\alpha \beta}}{2 \sqrt{-g}} \frac{\partial g_{\alpha \beta}}{\partial x_{a}^{i}}\right)_{b} \delta_{a b}
$$

see the box on the derivatives of the metric tensor determinant. 


\section{Derivatives of the metric tensor determinant $g$}

We will collect here some formulae related to the quantity $g$ that are needed in several places. The derivative of the metric tensor determinant is generally given by (see e.g. [228])

$$
\frac{\partial g}{\partial x^{\mu}}=g g^{\alpha \beta} \frac{\partial g_{\alpha \beta}}{\partial x^{\mu}}
$$

Therefore

$$
\frac{\partial \sqrt{-g}}{\partial x^{i}}=\frac{\sqrt{-g}}{2} g^{\alpha \beta} \frac{\partial g_{\alpha \beta}}{\partial x^{i}}, \quad \frac{\partial}{\partial x^{i}}\left(\frac{1}{\sqrt{-g}}\right)=-\frac{g^{\alpha \beta}}{2 \sqrt{-g}} \frac{\partial g_{\alpha \beta}}{\partial x^{i}}
$$

and

$$
\frac{d}{d t}(\sqrt{-g})=\frac{\partial}{\partial x^{\mu}}(\sqrt{-g}) \frac{d x^{\mu}}{d t}=\frac{\sqrt{-g}}{2} g^{\alpha \beta} \frac{\partial g_{\alpha \beta}}{\partial x^{u}} v^{\mu}=\frac{\sqrt{-g}}{2} g^{\alpha \beta} \frac{d g_{\alpha \beta}}{d t} .
$$

Of course, if the metric tensor at the position of particle $b$ is to be differentiated with respect to a property of particle $a$, the appropriate Kronecker deltas, $\delta_{a b}$, have to be applied.

With the help of Eqs. (202), (203), (204) and (207) Eq. (199) becomes

$$
\begin{aligned}
& \frac{\partial L}{\partial x_{a}^{i}}=+\sum_{b} \nu_{b}[1\left.+u_{b}\right]\left(\frac{U^{\mu} U^{\nu}}{2 \Theta}\right)_{b}\left(\frac{\partial g_{\mu \nu}}{\partial x_{a}^{i}}\right)_{b} \delta_{a b} \\
&-\sum_{b} \frac{\nu_{b}}{\Theta_{b}}\left\{\frac{P_{b}}{n_{b}^{2} \sqrt{-g_{b}} \Theta_{b}} \sum_{k} \nu_{k} \frac{\partial W_{b k}}{\partial x_{b}^{i}}\left(\delta_{b a}-\delta_{k a}\right)\right. \\
&+ \frac{P_{b} N_{b}^{*}}{n_{b}^{2} \sqrt{-g}_{b}}\left[-\left(\frac{U^{\mu} U^{\nu}}{2 \Theta}\right)_{b}\left(\frac{\partial g_{\mu \nu}}{\partial x_{a}^{i}}\right)_{b} \delta_{a b}\right] \\
&\left.+\frac{P_{b} N_{b}^{*}}{n_{b}^{2} \Theta_{b}}\left[-\left(\frac{g^{\mu \nu}}{2 \sqrt{-g}} \frac{\partial g_{\mu \nu}}{\partial x_{a}^{i}}\right)_{b} \delta_{a b}\right]\right\} .
\end{aligned}
$$

The terms that involve the derivatives of the kernel represent the hydrodynamic part of the equations, the others the action of gravity. After eliminating one sum in the hydrodynamic terms via the Kronecker symbol, relabeling the summation index from $k$ to $b$ and using kernel property Eq. (26) they read

$$
\left(\frac{\partial L}{\partial x_{a}^{i}}\right)_{\mathrm{h}}=-\nu_{a} \sum_{b} \nu_{b}\left(\frac{P_{a}}{n_{a}^{2} \sqrt{-g_{a}} \Theta_{a}^{2}}+\frac{P_{b}}{n_{b}^{2} \sqrt{-g_{b}} \Theta_{b}^{2}}\right) \frac{\partial W_{a b}}{\partial x_{a}^{i}} .
$$

and on using Eq. (187) 


$$
\left(\frac{\partial L}{\partial x_{a}^{i}}\right)_{\mathrm{h}}=-\nu_{a} \sum_{b} \nu_{b}\left(\frac{\sqrt{-g} P_{a}}{N_{a}^{* 2}}+\frac{\sqrt{-g_{b}} P_{b}}{N_{b}^{* 2}}\right) \frac{\partial W_{a b}}{\partial x_{a}^{i}} .
$$

The terms that involve derivatives of the metric ("gravity terms") can be written as

$$
\begin{aligned}
\left(\frac{\partial L}{\partial x_{a}^{i}}\right)_{\mathrm{g}}= & \frac{\nu_{a}}{2 \Theta_{a}}\left[\left(1+u_{a}+\frac{P_{a}}{n_{a}^{2}} \frac{N_{a}^{*}}{\sqrt{-g_{a}} \Theta_{a}}\right) U^{\mu} U^{\nu}+\frac{P_{a}}{n_{a}^{2}} \frac{N_{a}^{*}}{\sqrt{-g_{a}} \Theta_{a}} g^{\mu \nu}\right] \\
& \left(\frac{\partial g_{\mu \nu}}{\partial x^{i}}\right)_{a} .
\end{aligned}
$$

If we apply once more Eq. (187) and remember the form of the energymomentum tensor of the fluid, Eq. (181), the above term simply becomes

$$
\left(\frac{\partial L}{\partial x_{a}^{i}}\right)_{\mathrm{g}}=\frac{\nu_{a} \sqrt{-g}_{a}}{2 N_{a}^{*}}\left(T^{\mu \nu} \frac{\partial g_{\mu \nu}}{\partial x^{i}}\right)_{a}
$$

Putting all together, the spatial derivative of the Lagrangian reads

$$
\frac{\partial L}{\partial x_{a}^{i}}=\nu_{a}\left\{-\sum_{b} \nu_{b}\left(\frac{\sqrt{-g}_{a} P_{a}}{N_{a}^{* 2}}+\frac{\sqrt{-g}_{b} P_{b}}{N_{b}^{* 2}}\right) \frac{\partial W_{a b}}{\partial x_{a}^{i}}+\frac{\sqrt{-g}}{2 N_{a}^{*}}\left(T^{\mu \nu} \frac{\partial g_{\mu \nu}}{\partial x^{i}}\right)_{a}\right\} .
$$

Note that the first, hydrodynamical term has the symmetry in the particle indices, $a$ and $b$, that we know from previous forms of SPH equations, while the gravity term is exclusively evaluated at the position of the particle under consideration, $a$.

Inserting Eqs. (197), (198) and (214) into the Euler-Lagrange equations yields the final, general-relativistic momentum equation

$$
\frac{d S_{i, a}}{d t}=-\sum_{b} \nu_{b}\left(\frac{\sqrt{-g}_{a} P_{a}}{N_{a}^{* 2}}+\frac{\sqrt{-g}_{b} P_{b}}{N_{b}^{* 2}}\right) \frac{\partial W_{a b}}{\partial x_{a}^{i}}+\frac{\sqrt{-g} a}{2 N_{a}^{*}}\left(T^{\mu \nu} \frac{\partial g_{\mu \nu}}{\partial x^{i}}\right)_{a}
$$

\subsubsection{The energy equation}

We use once more guidance from the canonical energy to choose the numerical energy variable:

$$
\begin{aligned}
E & \equiv \sum_{a} \frac{\partial L}{\partial \vec{v}_{a}} \cdot \vec{v}_{a}-L=\sum_{a} \nu_{a} S_{i, a} v_{a}^{i}+\frac{\nu_{a}}{\Theta_{a}}\left[1+u_{a}\right] \\
& =\sum_{a} \nu_{a}\left[\Theta_{a}\left(1+u_{a}+\frac{P_{a}}{n_{a}}\right)\left(g_{i \mu} v^{\mu} v^{i}\right)_{a}+\frac{1+u_{a}}{\Theta_{a}}\right]=\sum_{a} \nu_{a} \hat{e}_{a}
\end{aligned}
$$


Here we have introduced the energy per baryon

$$
\hat{e}_{a} \equiv \Theta_{a}\left(1+u_{a}+\frac{P_{a}}{n_{a}}\right)\left(g_{i \mu} v^{\mu} v^{i}\right)_{a}+\frac{1+u_{a}}{\Theta_{a}}=S_{i, a} v_{a}^{i}+\frac{1+u_{a}}{\Theta_{a}},
$$

very similar to the special-relativistic energy variable, Eq. (161). Its temporal change is given by

$$
\frac{d \hat{e}_{a}}{d t}=\frac{d S_{i, a}}{d t} v_{a}^{i}+S_{i, a} \frac{d v_{a}^{i}}{d t}+\frac{d}{d t}\left(\frac{1+u_{a}}{\Theta_{a}}\right)
$$

The first time derivative is known from the momentum equation, the second can be hoped to cancel out with a term resulting from the last term, as in the special-relativistic case, see Eq. (164). For this last term we need $d u_{a} / d t$ and $d \Theta_{a} / d t$. To obtain the time derivative of $u_{a}$ we use the first law of thermodynamics, Eq. (8), the number density relation between the frames, Eq. (187), and $d(\sqrt{-g}) / d t$, Eq. (208). This delivers

$$
\frac{d u_{a}}{d t}=\frac{P_{a}}{n_{a}^{2}} \frac{d}{d t}\left(\frac{N^{*}}{\sqrt{-g} \Theta}\right)_{a}=\frac{P_{a} \sqrt{-g} \Theta_{a}}{N_{a}^{* 2}} \frac{d N_{a}^{*}}{d t}+\frac{P_{a} N_{a}^{*}}{n_{a}^{2}} \frac{d}{d t}\left(\frac{1}{\sqrt{-g} \Theta}\right)_{a} .
$$

The last derivative is given by

$$
\frac{d}{d t}\left(\frac{1}{\sqrt{-g} \Theta}\right)_{a}=-\left(\frac{1}{2 \sqrt{-g} \Theta} g^{\alpha \beta} \frac{d g_{\alpha \beta}}{d t}\right)_{a}-\left(\frac{1}{\sqrt{-g} \Theta^{2}} \frac{d \Theta}{d t}\right)_{a},
$$

thus the change in $u_{a}$ becomes

$$
\frac{d u_{a}}{d t}=\frac{P_{a} \sqrt{-g} \Theta_{a}}{N_{a}^{* 2}} \frac{d N_{a}^{*}}{d t}-\frac{P_{a}}{2 n_{a}} g^{\alpha \beta} \frac{d g_{\alpha \beta}}{d t}-\frac{P_{a}}{n_{a} \Theta_{a}} \frac{d \Theta_{a}}{d t} .
$$

It remains to calculate the derivative of the generalized Lorentz-factor

$$
\frac{d \Theta_{a}}{d t}=\frac{d}{d t}\left(-g_{\alpha \beta} v^{\alpha} v^{\beta}\right)_{a}^{-1 / 2}=\left(\frac{\Theta^{3}}{2} v^{\alpha} v^{\beta} \frac{d g_{\alpha \beta}}{d t}+\Theta^{3} g_{\alpha \beta} \frac{d v^{\alpha}}{d t} v^{\beta}\right)_{a}
$$

On using Eqs. (221) and (222) we find

$$
\begin{aligned}
\frac{d}{d t}\left(\frac{1+u_{a}}{\Theta_{a}}\right)= & \frac{1}{\Theta_{a}}\left\{\frac{P_{a} \sqrt{-g_{a}} \Theta_{a}}{N_{a}^{* 2}} \frac{d N_{a}^{*}}{d t}-\frac{P_{a}}{2 n_{a}} g^{\alpha \beta} \frac{d g_{\alpha \beta}}{d t}-\frac{P_{a}}{n_{a} \Theta_{a}} \frac{d \Theta}{d t}\right\} \\
& -\frac{1+u_{a}}{\Theta_{a}^{2}} \frac{d \Theta}{d t}
\end{aligned}
$$




$$
\begin{aligned}
= & \frac{P_{a} \sqrt{-g}}{N_{a}^{* 2}} \frac{d N_{a}^{*}}{d t}-\Theta_{a}\left(1+u_{a}+\frac{P_{a}}{n_{a}}\right)\left(g_{\alpha \beta} v^{\alpha} \frac{d v^{\beta}}{d t}\right)_{a} \\
& -\left(\frac{P_{a}}{2 n_{a} \Theta_{a}} g_{a}^{\alpha \beta}+\frac{\Theta_{a}}{2}\left[1+u_{a}+\frac{P_{a}}{n_{a}}\right] v^{\alpha} v^{\beta}\right)\left(\frac{d g_{\alpha \beta}}{d t}\right)_{a}
\end{aligned}
$$

If we replace the velocities via Eq. (184) by four-velocities and use the definition of the energy momentum tensor, Eq. (181), the equation simplifies to

$$
\begin{aligned}
\frac{d}{d t}\left(\frac{1+u_{a}}{\Theta_{a}}\right)= & \frac{P_{a} \sqrt{-g}}{N_{a}^{* 2}} \frac{d N_{a}^{*}}{d t}-\Theta_{a}\left(1+u_{a}+\frac{P_{a}}{n_{a}}\right)\left(g_{\alpha \beta} v^{\alpha} \frac{d v^{\beta}}{d t}\right)_{a} \\
& -\left(\frac{\sqrt{-g} T^{\alpha \beta}}{2 N^{*}} \frac{d g_{\alpha \beta}}{d t}\right)_{a} \\
= & \frac{P_{a} \sqrt{-g}}{N_{a}^{* 2}} \frac{d N_{a}^{*}}{d t}-\left(S_{i}\right)_{a} \frac{d v_{a}^{i}}{d t}-\left(\frac{\sqrt{-g} T^{\alpha \beta}}{2 N^{*}} \frac{d g_{\alpha \beta}}{d t}\right)_{a} .
\end{aligned}
$$

Here we have split the contraction in the term containing $d v^{\beta} / d t$ into a spatial and a temporal part and we made use of $v^{0}=1$, see Eq. (184). This equation is very similar the special-relativistic one, Eq. (164), but it also contains a gravity contribution. If we insert Eq. (224) into the energy evolution equation (218), use the SPH prescription for $d N^{*} / d t$ and collect terms into "hydro" (kernels) and "gravity" (metric derivatives) we find

$$
\begin{aligned}
\frac{d \hat{e}_{a}}{d t} & =-\sum_{b} \nu_{b}\left(\frac{\sqrt{-g_{a}} P_{a} \vec{v}_{b}}{N_{a}^{* 2}}+\frac{\sqrt{-g_{b}} P_{b} \vec{v}_{a}}{N_{b}^{* 2}}\right) \cdot \nabla_{a} W_{a b}+\frac{\sqrt{-g}}{2 N_{a}^{*}} T_{a}^{\alpha \beta}\left(\frac{\partial g_{\alpha \beta}}{\partial x^{i}} v^{i}-\frac{d g_{\alpha \beta}}{d t}\right)_{a} \\
& =-\sum_{b} \nu_{b}\left(\frac{\sqrt{-g_{a}} P_{a} \vec{v}_{b}}{N_{a}^{* 2}}+\frac{\sqrt{-g_{b}} P_{b} \vec{v}_{a}}{N_{b}^{* 2}}\right) \cdot \nabla_{a} W_{a b}-\frac{\sqrt{-g}}{2 N_{a}^{*}}\left(T^{\alpha \beta} \frac{\partial g_{\alpha \beta}}{\partial t}\right)_{a}
\end{aligned}
$$

This is the final, general-relativistic SPH energy equation. The equations (191), (215), and (225) together with an equation of state form the set of general-relativistic SPH equations in a fixed background metric. 


\section{Summary of the general-relativistic SPH equations on a fixed background metric}

Ignoring derivatives from the smoothing lengths, the momentum equation reads

$$
\frac{d S_{i, a}}{d t}=-\sum_{b} \nu_{b}\left(\frac{\sqrt{-g}_{a} P_{a}}{N_{a}^{* 2}}+\frac{\sqrt{-g}_{b} P_{b}}{N_{b}^{* 2}}\right) \frac{\partial W_{a b}}{\partial x_{a}^{i}}+\frac{\sqrt{-g} a}{2 N_{a}^{*}}\left(T^{\mu \nu} \frac{\partial g_{\mu \nu}}{\partial x^{i}}\right)_{a}
$$

where

$$
S_{i, a}=\Theta_{a}\left(1+u_{a}+\frac{P_{a}}{n_{a}}\right)\left(g_{i \mu} v^{\mu}\right)_{a}
$$

is the canonical momentum per baryon and

$$
\Theta_{a}=\left(-g_{\mu \nu} v^{\mu} v^{\nu}\right)_{a}^{-\frac{1}{2}}
$$

the generalized Lorentz factor. The energy equation reads

$\frac{d \hat{\epsilon}_{a}}{d t}=-\sum_{b} \nu_{b}\left(\frac{\sqrt{-g_{a}} P_{a}}{N_{a}^{* 2}} \vec{v}_{b}+\frac{\sqrt{-g}_{b} P_{b}}{N_{b}^{* 2}} \vec{v}_{a}\right) \cdot \nabla_{a} W_{a b}-\frac{\sqrt{-g}}{2 N_{a}^{*}}\left(T^{\mu \nu} \frac{\partial g_{\mu \nu}}{\partial t}\right)_{a}$

where

$$
\hat{\epsilon}_{a}=S_{i, a} v_{a}^{i}+\frac{1+u_{a}}{\Theta_{a}}
$$

is the canonical energy per nucleon. The number density can again be calculated via summation,

$$
N_{a}^{*}=\sum_{b} \nu_{b} W_{a b}\left(h_{a}\right)
$$

\section{Summary}

Since the Smooth Particle Hydrodynamics method was suggested in the late seventies it has undergone a long sequence of technical improvements. In parallel, the method has been applied to a large variety of problems both inside and outside astrophysics, and consequently a slew of different physical processes has been included in SPH-based simulations.

In this review we have only very briefly touched upon this latter development,

we merely provided pointers to the current literature. Instead we have focused 
on what we hope is a pedagogical introduction leading to an in-depth understanding of how the method works. All essential equations were derived, and in particular, a commonly used ("vanilla ice") set of SPH equations that directly discretizes the Lagrangian equations of an ideal fluid. We have also reviewed concepts such as adaptive numerical resolution, the reasoning behind artificial viscosity and basic aspects of the ODE integration in the SPH context.

The "vanilla ice" equation set works well in practice and possesses the property of "hard-wired" conservation of mass, energy, linear and angular momentum. The symmetrization in the particle indices is, however, somewhat arbitrary and was enforced ad hoc. In Sec. 3, a modern form of the SPH equations was derived that improves on this weakness: it uses nothing more than the Lagrangian of an ideal fluid, the first law of thermodynamics and a prescription how to obtain the density by a summation over particles. After the Lagrangian has been written in its SPH-discrete form, there is no more arbitrariness left in the rest of the derivation: the equations follow stringently from the EulerLagrange equations. In addition to curing the aesthetical shortcoming of the previous approach, the latter also naturally leads to corrective terms due to the derivatives of the smoothing lengths, in SPH usually called "grad-h-terms".

The elegant variational concept naturally carries over to both the special- and general-relativistic (fixed-metric) case. Here, we derived for the first time the special-relativistic SPH equations that include "grad-h-terms". The numerical variables are chosen as canonical momentum and energy per baryon. While this has obvious numerical advantages, it comes at the price of recovering the physical variables from the numerical ones at each time step, a tribute that is also payed in modern grid-based relativistic approaches, see e.g. [226]. The performance of this new equation set has been briefly illustrated at the example of a relativistic shock tube test, for a more exhaustive set of tests we refer to $[227]$.

We conclude this review with a derivation of the general-relativistic case in which the space-time metric can be considered unperturbed by the self-gravity of the fluid, a case that is realized, for example, in the tidal disruption of a star by a supermassive black hole. The resulting equations are a natural generalization of the special-relativistic case and do not introduce much of an additional numerical burden. The resulting extra terms can be analytically calculated from the metric that is assumed to be known.

In the future, more physical processes and their corresponding numerical schemes will certainly find their ways into SPH. On the numerical hydrodynamics side the recent past has seen a couple suggestions on how to improve on known weaknesses of SPH. One of them was SPH's unsatisfactory performance on fluid instabilities across discontinuities with large density jumps [185]. A modification of artificial dissipation terms [154] or, alternatively, a more general formulation of the SPH equations [162] have been suggested as cures for this problem. In terms of artificial dissipation, a tensor artificial viscosity approach such as the one suggested in [175] should definitely be explored further for its potential and usability in astrophysics. While the special- 
relativistic SPH equations as derived in Sec. 4 have been carefully tested, the general-relativistic equation set has so far not been implemented and applied to astrophysical problems. This task is left for future investigations.

\section{Acknowledgments}

It is a great pleasure to thank Marcus Brüggen, Marius Dan, James Guillochon and Roland Speith for their careful reading of the manuscript.

\section{References}

[1] L. Lucy, A numerical approach to the testing of the fission hypothesis, The Astronomical Journal 82 (1977) 1013.

[2] R. A. Gingold, J. J. Monaghan, Smoothed particle hydrodynamics - Theory and application to non-spherical stars, MNRAS 181 (1977) 375-389.

[3] L. Hernquist, N. Katz, Treesph - a unification of sph with the hierarchical tree method, ApJS 70 (1989) 419.

[4] W. Benz, Smooth particle hydrodynamics: A review, in: J. Buchler (Ed.), Numerical Modeling of Stellar Pulsations, Kluwer Academic Publishers, Dordrecht, 1990, p. 269.

[5] J. J. Monaghan, Smoothed particle hydrodynamics, Ann. Rev. Astron. Astrophys. 30 (1992) 543.

[6] J. J. Monaghan, Smoothed particle hydrodynamics, Reports on Progress in Physics 68 (2005) 1703-1759.

[7] J. Barnes, P. Hut, A Hierarchical O(NlogN) Force-Calculation Algorithm, Nature 324 (1986) 446-449.

[8] M. S. Warren, J. K. Salmon, A parallel, portable and versatile treecode., in: Parallel Processing for Scientific Computing, 1995, pp. 319-324.

[9] R. Dave, J. Dubinski, L. Hernquist, Parallel TreeSPH, New Astronomy 2 (1997) 277-297.

[10] G. Carraro, C. Lia, C. Chiosi, Galaxy formation and evolution - I. The Padua tree-sph code (pd-sph), MNRAS 297 (1998) 1021-1040.

[11] V. Springel, N. Yoshida, S. D. M. White, GADGET: a code for collisionless and gasdynamical cosmological simulations, New Astronomy 6 (2001) 79-117.

[12] V. Springel, The cosmological simulation code GADGET-2, MNRAS 364 (2005) 1105-1134.

[13] W. Benz, R. Bowers, A. Cameron, W. Press, Dynamic mass exchange in doubly degenerate binaries. i - 0.9 and 1.2 solar mass stars, ApJ 348 (1990) 647. 
[14] J. W. Wadsley, J. Stadel, T. Quinn, Gasoline: a flexible, parallel implementation of TreeSPH, New Astronomy 9 (2004) 137-158.

[15] A. F. Nelson, M. Wetzstein, T. Naab, ., VINE - A numerical code for simulating astrophysical systems using particles II: Implementation and performance characteristics, ArXiv e-prints.

[16] R. W. Hockney, J. W. Eastwood, Computer Simulation Using Particles, 1st Edition, McGraw-Hill, New York, 1988.

[17] A. Klypin, J. Holtzman, Particle-Mesh code for cosmological simulations, ArXiv Astrophysics e-prints.

[18] J. Dubinski, J. Kim, C. Park, R. Humble, GOTPM: a parallel hybrid particlemesh treecode, New Astronomy 9 (2004) 111-126.

[19] W. Dehnen, A Very Fast and Momentum-conserving Tree Code, ApJL 536 (2000) L39-L42.

[20] W. Dehnen, A Hierarchical $\mathrm{O}(\mathrm{N})$ Force Calculation Algorithm, Journal of Computational Physics 179 (2002) 27-42.

[21] L. Greengard, V. Rokhlin, A fast algorithm for particle simulations, Journal of Computational Physics 73 (1987) 325-348.

[22] T. Theuns, Parallel P3M with exact calculation of short range forces, Computer Physics Communications 78 (1994) 238-246.

[23] T. Theuns, A. Leonard, G. Efstathiou, F. R. Pearce, P. A. Thomas, P $\mathrm{P}^{3} \mathrm{M}-\mathrm{SPH}$ simulations of the Lyalpha forest, MNRAS 301 (1998) 478-502.

[24] P. Bode, J. P. Ostriker, G. Xu, The Tree Particle-Mesh N-Body Gravity Solver, ApJS 128 (2000) 561-569.

[25] A. Knebe, A. Green, J. Binney, Multi-level adaptive particle mesh (MLAPM): a c code for cosmological simulations, MNRAS 325 (2001) 845-864.

[26] P. Bode, J. P. Ostriker, Tree Particle-Mesh: An Adaptive, Efficient, and Parallel Code for Collisionless Cosmological Simulation, ApJS 145 (2003) 1-13.

[27] M. Herant, W. Benz, W. R. Hix, C. L. Fryer, S. A. Colgate, Inside the supernova: A powerful convective engine, ApJ 435 (1994) 339-361.

[28] C. Fryer, W. Benz, M. Herant, S. A. Colgate, What Can the Accretion-induced Collapse of White Dwarfs Really Explain?, ApJ 516 (1999) 892-899.

[29] S. Rosswog, M. Liebendörfer, F.-K. Thielemann, M. Davies, W. Benz, T. Piran, Mass ejection in neutron star mergers, A \& A 341 (1999) 499-526.

[30] S. Rosswog, M. B. Davies, High-resolution calculations of merging neutron stars - I. Model description and hydrodynamic evolution, MNRAS 334 (2002) $481-497$. 
[31] C. L. Fryer, G. Rockefeller, M. S. Warren, SNSPH: A Parallel Threedimensional Smoothed Particle Radiation Hydrodynamics Code, ApJ 643 (2006) 292-305.

[32] J. Guerrero, E. Garcia-Berro, J. Isern, Smoothed Particle Hydrodynamics simulations of merging white dwarfs, A\&A 413 (2004) 257-272.

[33] W. H. Lee, E. Ramirez-Ruiz, D. Page, Opaque or Transparent? A Link between Neutrino Optical Depths and the Characteristic Duration of Short GammaRay Bursts, ApJL 608 (2004) L5-L8.

[34] W. H. Lee, E. Ramirez-Ruiz, D. Page, Dynamical Evolution of Neutrino-cooled Accretion Disks: Detailed Microphysics, Lepton-driven Convection, and Global Energetics, ApJ 632 (2005) 421-437.

[35] S. Yoon, P. Podsiadlowski, S. Rosswog, Remnant evolution after a carbonoxygen white dwarf merger, MNRAS 380 (2007) 933.

[36] S. Rosswog, E. Ramirez-Ruiz, W. R. Hix, M. Dan, Simulating black hole white dwarf encounters, Computer Physics Communications 179 (2008) 184-189.

[37] L. D. Libersky, A. G. Petschek, Smooth Particle Hydrodynamics with Stength of Materials, in: H. E. Trease, M. J. Fritts, W. P. Crowley (Eds.), Advances in the Free-Lagrange Method, Springer Verlag, 1990, pp. 248-257.

[38] L. D. Libersky, A. G. Petschek, T. C. Carney, J. R. Hipp, F. A. Allahdadi, High Strain Lagrangian Hydrodynamics.

[39] W. Benz, E. Asphaug, Impact simulations with fracture. I - Method and tests, Icarus 107 (1994) 98.

[40] W. Benz, E. Asphaug, Simulations of brittle solids using smooth particle hydrodynamics, Computer Physics Communications 87 (1995) 253-265.

[41] E. Asphaug, W. Benz, Size, Density, and Structure of Comet Shoemaker-Levy 9 Inferred from the Physics of Tidal Breakup, Icarus 121 (1996) 225-248.

[42] P. W. Randles, L. D. Libersky, Smoothed Particle Hydrodynamics: Some recent Improvements and Applications, Comp. Methods Appl. Mech. Engrg. 139 (1996) 375-408.

[43] D. A. Hicks, J. W. Swegle, S. W. Attaway, Conservative Smoothing Stabilizes Discrete-Numerical Instabilities in SPH Material Dynamics Computations, Applied Mathematics and Computation 85 (1997) 209-226.

[44] R. Vignjevic, J. Campbell, L. Libersky, A treatment of zero-energy modes in the smoothed particle hydrodynamics method, Comp. Methods Appl. Mech. Engrg. 184 (2000) 67-85.

[45] C. Schäfer, R. Speith, W. Kley, Collisions between equal-sized ice grain agglomerates, A \& A 470 (2007) 733-739.

[46] S. Sirono, Conditions for collisional growth of a grain aggregate, Icarus 167 (2004) 431-452. 
[47] M. Jutzi, W. Benz, P. Michel, Numerical simulations of impacts involving porous bodies. I. Implementing sub-resolution porosity in a $3 \mathrm{D} \mathrm{SPH}$ hydrocode, Icarus 198 (2008) 242-255.

[48] J. Frank, A. King, D. J. Raine, Accretion Power in Astrophysics: Third Edition, Accretion Power in Astrophysics, by Juhan Frank and Andrew King and Derek Raine, pp. 398. ISBN 0521620538. Cambridge, UK: Cambridge University Press, February 2002., 2002.

[49] A. C. Fabian, J. S. Sanders, C. S. Crawford, C. J. Conselice, J. S. Gallagher, R. F. G. Wyse, The relationship between the optical $\mathrm{H} \alpha$ filaments and the Xray emission in the core of the Perseus cluster, MNRAS 344 (2003) L48-L52.

[50] O. Flebbe, S. Muenzel, H. Herold, H. Riffert, H. Ruder, Smoothed Particle Hydrodynamics: Physical viscosity and the simulation of accretion disks, ApJ 431 (1994) 754-760.

[51] H. Takeda, S. M. Miyama, M. Sekiya, Numerical Simulation of Viscous Flow by Smoothed Particle Hydrodynamics, Progress of Theoretical Physics 92 (5) (1994) 939-960.

[52] S. Watkins, A. Bhattal, N. Francis, J. Turner, A. Whitworth, A new prescription for viscosity in Smoothed Particle Hydrodynamics, A\&AS 119 (1996) 177.

[53] J. Morris, J. Monaghan, A switch to reduce sph viscosity, J. Comp. Phys. 136 (1997) 41.

[54] W. H. Lee, E. Ramirez-Ruiz, Accretion Disks around Black Holes: Dynamical Evolution, Meridional Circulations, and Gamma-Ray Bursts, ApJ 577 (2002) 893-903.

[55] C. Schäfer, R. Speith, R. Günther, W. Kley, Simulations of Planet-Disc Interactions with SPH, Astronomische Nachrichten Supplement 325 (2004) 85 .

[56] D. Sijacki, V. Springel, Physical viscosity in smoothed particle hydrodynamics simulations of galaxy clusters, MNRAS 371 (2006) 1025-1046.

[57] L. Brookshaw, A method of calculating radiative heat diffusion in particle simulations, Proceedings of the Astronomical Society of Australia 6 (1985) $207-210$.

[58] P. W. Cleary, J. J. Monaghan, Conduction Modelling Using Smoothed Particle Hydrodynamics, Journal of Computational Physics 148 (1999) 227-264.

[59] M. Jubelgas, V. Springel, K. Dolag, Thermal conduction in cosmological SPH simulations, MNRAS 351 (2004) 423-435.

[60] W. Benz, F.-K. Thielemann, J. G. Hills, Three-dimensional hydrodynamical simulations of stellar collisions. II - White dwarfs, ApJ 342 (1989) 986-998. 
[61] W. R. Hix, A. M. Khokhlov, J. C. Wheeler, F.-K. Thielemann, The QuasiEquilibrium-reduced alpha -Network, ApJ 503 (1998) 332.

[62] F. X. Timmes, Integration of Nuclear Reaction Networks for Stellar Hydrodynamics, ApJS 124 (1999) 241-263.

[63] M. Dan, S. Rosswog, M. Brüggen, Mass transfer dynamics in double degenerate binary systems, Journal of Physics Conference Series 172 (1) (2009) 012034-+.

[64] S. Rosswog, D. Kasen, J. Guillochon, E. Ramirez-Ruiz, Collisions of white dwarfs as a new progenitor channel for type Ia supernovae, ArXiv e-prints.

[65] C. Raskin, F. X. Timmes, E. Scannapieco, S. Diehl, C. Fryer, On Type Ia Supernovae From The Collisions of Two White Dwarfs, ArXiv e-prints.

[66] S. Rosswog, E. Ramirez-Ruiz, R. Hix, Atypical thermonuclear supernovae from tidally crushed white dwarfs, ApJ 679 (2008) 1385.

[67] S. Rosswog, E. Ramirez-Ruiz, R. Hix, Tidal disruption and ignition of white dwarfs by moderately massive black holes, ApJ 695 (2009) 404-419.

[68] M. Steinmetz, E. Mueller, The formation of disk galaxies in a cosmological context: Populations, metallicities and metallicity gradients, A \& A 281 (1994) L97-L100.

[69] M. Steinmetz, E. Muller, The formation of disc galaxies in a cosmological context: structure and kinematics, MNRAS 276 (1995) 549-562.

[70] C. M. Raiteri, M. Villata, J. F. Navarro, Simulations of Galactic chemical evolution. I. O and Fe abundances in a simple collapse model., A \& A 315 (1996) 105-115.

[71] G. Carraro, Y. K. Ng, L. Portinari, On the Galactic disc age-metallicity relation, MNRAS 296 (1998) 1045-1056.

[72] P. Berczik, Chemo-dynamical smoothed particle hydrodynamic code for evolution of star forming disk galaxies, A \& A 348 (1999) 371-380.

[73] D. Kawata, B. K. Gibson, GCD+: a new chemodynamical approach to modelling supernovae and chemical enrichment in elliptical galaxies, MNRAS 340 (2003) 908-922.

[74] C. Kobayashi, GRAPE-SPH chemodynamical simulation of elliptical galaxies - I. Evolution of metallicity gradients, MNRAS 347 (2004) 740-758.

[75] L. Tornatore, S. Borgani, F. Matteucci, S. Recchi, P. Tozzi, Simulating the metal enrichment of the intracluster medium, MNRAS 349 (2004) L19-L24.

[76] C. Scannapieco, P. B. Tissera, S. D. M. White, V. Springel, Feedback and metal enrichment in cosmological smoothed particle hydrodynamics simulations - I. A model for chemical enrichment, MNRAS 364 (2005) 552-564.

[77] F. J. Martinez-Serrano, A. Serna, R. Dominguez-Tenreiro, M. Molla, Chemical evolution of galaxies - I. A composition-dependent SPH model for chemical evolution and cooling, MNRAS 388 (2008) 39-55. 
[78] T. H. Greif, S. C. O. Glover, V. Bromm, R. S. Klessen, Chemical mixing in smoothed particle hydrodynamics simulations, MNRAS 392 (2009) 1381-1387.

[79] R. P. C. Wiersma, J. Schaye, T. Theuns, C. Dalla Vecchia, L. Tornatore, Chemical enrichment in cosmological, smoothed particle hydrodynamics simulations, ArXiv e-prints.

[80] N. Katz, D. H. Weinberg, L. Hernquist, Cosmological Simulations with TreeSPH, ApJS 105 (1996) 19.

[81] S. Viau, P. Bastien, S.-H. Cha, An Implicit Method for Radiative Transfer with the Diffusion Approximation in Smooth Particle Hydrodynamics, ApJ 639 (2006) 559-570.

[82] S. C. Whitehouse, M. R. Bate, J. J. Monaghan, A faster algorithm for smoothed particle hydrodynamics with radiative transfer in the flux-limited diffusion approximation, MNRAS 364 (2005) 1367-1377.

[83] S. C. Whitehouse, M. R. Bate, The thermodynamics of collapsing molecular cloud cores using smoothed particle hydrodynamics with radiative transfer, MNRAS 367 (2006) 32-38.

[84] L. Mayer, G. Lufkin, T. Quinn, J. Wadsley, Fragmentation of Gravitationally Unstable Gaseous Protoplanetary Disks with Radiative Transfer, ApJl 661 (2007) L77-L80.

[85] S. Oxley, M. M. Woolfson, Smoothed particle hydrodynamics with radiation transfer, MNRAS 343 (2003) 900-912.

[86] D. Stamatellos, A. P. Whitworth, Monte Carlo radiative transfer in SPH density fields, A \& A 439 (2005) 153-158.

[87] B. Semelin, F. Combes, S. Baek, Lyman-alpha radiative transfer during the epoch of reionization: contribution to 21-cm signal fluctuations, A \& A 474 (2007) 365-374.

[88] R. A. C. Croft, G. Altay, Radiation-induced large-scale structure during the reionization epoch: the autocorrelation function, MNRAS 388 (2008) 15011520 .

[89] O. Kessel-Deynet, A. Burkert, Ionizing radiation in smoothed particle hydrodynamics, MNRAS 315 (2000) 713-721.

[90] M. A. Alvarez, V. Bromm, P. R. Shapiro, The H II Region of the First Star, ApJ 639 (2006) 621-632.

[91] G. Altay, R. A. C. Croft, I. Pelupessy, SPHRAY: a smoothed particle hydrodynamics ray tracer for radiative transfer, MNRAS 386 (2008) 19311946.

[92] J. E. Dale, B. Ercolano, C. J. Clarke, A new algorithm for modelling photoionizing radiation in smoothed particle hydrodynamics, MNRAS 382 (2007) 1759-1767. 
[93] J. L. Johnson, T. H. Greif, V. Bromm, Local Radiative Feedback in the Formation of the First Protogalaxies, ApJ 665 (2007) 85-95.

[94] H. Susa, Smoothed Particle Hydrodynamics Coupled with Radiation Transfer, Publications of the Astronomical Society of Japan 58 (2006) 445-460.

[95] A. H. Pawlik, J. Schaye, TRAPHIC - radiative transfer for smoothed particle hydrodynamics simulations, MNRAS 389 (2008) 651-677.

[96] M. Gritschneder, T. Naab, A. Burkert, S. Walch, F. Heitsch, M. Wetzstein, iVINE - Ionization in the parallel TREE/SPH code VINE: first results on the observed age-spread Around O-stars, MNRAS 393 (2009) 21-31.

[97] D. Forgan, K. Rice, D. Stamatellos, A. Whitworth, Introducing a hybrid radiative transfer method for smoothed particle hydrodynamics, MNRAS (2009) $172-+$.

[98] J. R. Wilson, in: J. M. Centrella, J. M. LeBlanc, R. L. Bowers (Eds.), Numerical Astrophysics, Jones and Bartlett, Boston, 1982, p. 422.

[99] H. A. Bethe, J. R. Wilson, Revival of a stalled supernova shock by neutrino heating, ApJ 295 (1985) 14-23.

[100] S. Rosswog, M. Liebendörfer, High-resolution calculations of merging neutron stars - II. Neutrino emission, MNRAS 342 (2003) 673-689.

[101] G. J. Phillips, J. J. Monaghan, A numerical method for three-dimensional simulations of collapsing, isothermal, magnetic gas clouds, MNRAS 216 (1985) $883-895$.

[102] K. Dolag, M. Bartelmann, H. Lesch, Evolution and structure of magnetic fields in simulated galaxy clusters, A\&A 387 (2002) 383-395.

[103] D. J. Price, J. J. Monaghan, Smoothed Particle Magnetohydrodynamics - I. Algorithm and tests in one dimension, MNRAS 348 (2004) 123-138.

[104] D. J. Price, J. J. Monaghan, Smoothed Particle Magnetohydrodynamics II. Variational principles and variable smoothing-length terms, MNRAS 348 (2004) 139-152.

[105] D. Price, Magnetic fields in astrophysics, Ph.D. thesis, University of Cambridge, arXiv:astro-ph/0507472 (2004).

[106] D. J. Price, J. J. Monaghan, Smoothed Particle Magnetohydrodynamics - III. Multidimensional tests and the $\nabla . \mathrm{B}=0$ constraint, MNRAS 364 (2005) 384406.

[107] S. Børve, M. Omang, J. Trulsen, Regularized Smoothed Particle Hydrodynamics: A New Approach to Simulating Magnetohydrodynamic Shocks, ApJ 561 (2001) 82-93.

[108] S. Børve, M. Omang, J. Trulsen, Two-dimensional MHD Smoothed Particle Hydrodynamics Stability Analysis, ApJS 153 (2004) 447-462. 
[109] S.

Børve,

M. Omang, J. Trulsen, Regularized smoothed particle hydrodynamics with improved multi-resolution handling, J. Comp. Phys. 208 (2005) 345-367.

[110] S. Børve, M. Omang, J. Trulsen, Multidimensional MHD Shock Tests of Regularized Smoothed Particle Hydrodynamics, ApJ 652 (2006) 1306-1317.

[111] K. Dolag, F. A. Stasyszyn, An MHD Gadget for cosmological simulations, ArXiv e-prints.

[112] S. Rosswog, D. Price, Magma: a magnetohydrodynamics code for merger applications, MNRAS 379 (2007) 915 - 931.

[113] S. Rosswog, D. Price, Meshless magnetohydrodynamics, Springer Lecture Notes in Computational Science and Engineering (2008) 247-275.

[114] T. A. Enßlin, C. Pfrommer, V. Springel, M. Jubelgas, Cosmic ray physics in calculations of cosmological structure formation, A \& A 473 (2007) 41-57.

[115] M. Jubelgas, V. Springel, T. Enßlin, C. Pfrommer, Cosmic ray feedback in hydrodynamical simulations of galaxy formation, A\&A 481 (2008) 33-63.

[116] C. Pfrommer, T. A. Enßlin, V. Springel, Simulating cosmic rays in clusters of galaxies - II. A unified scheme for radio haloes and relics with predictions of the $\gamma$-ray emission, MNRAS 385 (2008) 1211-1241.

[117] C. Pfrommer, Simulating cosmic rays in clusters of galaxies - III. Non-thermal scaling relations and comparison to observations, MNRAS 385 (2008) 12421256 .

[118] D. Sijacki, C. Pfrommer, V. Springel, T. A. Enßlin, Simulations of cosmicray feedback by active galactic nuclei in galaxy clusters, MNRAS 387 (2008) 1403-1415.

[119] T. Di Matteo, V. Springel, L. Hernquist, Energy input from quasars regulates the growth and activity of black holes and their host galaxies, NATURE 433 (2005) 604-607.

[120] V. Springel, T. Di Matteo, L. Hernquist, Modelling feedback from stars and black holes in galaxy mergers, MNRAS 361 (2005) 776-794.

[121] J. F. Navarro, S. D. M. White, Simulations of Dissipative Galaxy Formation in Hierarchically Clustering Universes - Part One - Tests of the Code, MNRAS 265 (1993) 271.

[122] J. C. Mihos, L. Hernquist, Star-forming galaxy models: Blending star formation into TREESPH, ApJ 437 (1994) 611-624.

[123] M. R. Bate, I. A. Bonnell, N. M. Price, Modelling accretion in protobinary systems, MNRAS 277 (1995) 362-376.

[124] J. P. E. Gerritsen, V. Icke, Star formation in N-body simulations. I. The impact of the stellar ultraviolet radiation on star formation., A \& A 325 (1997) 972986. 
[125] J. Sommer-Larsen, S. Gelato, H. Vedel, Formation of Disk Galaxies: Feedback and the Angular Momentum Problem, ApJ 519 (1999) 501-512.

[126] V. Springel, Modelling star formation and feedback in simulations of interacting galaxies, MNRAS 312 (2000) 859-879.

[127] V. Springel, M. White, L. Hernquist, Hydrodynamic Simulations of the Sunyaev-Zeldovich Effect(s), ApJ 549 (2001) 681-687.

[128] R. J. Thacker, H. M. P. Couchman, Implementing Feedback in Simulations of Galaxy Formation: A Survey of Methods, ApJ 545 (2000) 728-752.

[129] E. Scannapieco, R. J. Thacker, M. Davis, High-Redshift Galaxy Outflows and the Formation of Dwarf Galaxies, ApJ 557 (2001) 605-615.

[130] R. J. Thacker, H. M. P. Couchman, Star Formation, Supernova Feedback, and the Angular Momentum Problem in Numerical Cold Dark Matter Cosmogony: Halfway There?, ApJL 555 (2001) L17-L20.

[131] S. T. Kay, F. R. Pearce, C. S. Frenk, A. Jenkins, Including star formation and supernova feedback within cosmological simulations of galaxy formation, MNRAS 330 (2002) 113-128.

[132] Y. Ascasibar, G. Yepes, S. Gottlöber, V. Müller, Numerical simulations of the cosmic star formation history, A \& A 387 (2002) 396-405.

[133] T. Theuns, M. Viel, S. Kay, J. Schaye, R. F. Carswell, P. Tzanavaris, Galactic Winds in the Intergalactic Medium, ApJL 578 (2002) L5-L8.

[134] V. Springel, L. Hernquist, Cosmological smoothed particle hydrodynamics simulations: a hybrid multiphase model for star formation, MNRAS 339 (2003) $289-311$.

[135] M. R. Bate, I. A. Bonnell, V. Bromm, The formation of a star cluster: predicting the properties of stars and brown dwarfs, MNRAS 339 (2003) 577599 .

[136] I. A. Bonnell, M. R. Bate, S. G. Vine, The hierarchical formation of a stellar cluster, MNRAS 343 (2003) 413-418.

[137] S. Marri, S. D. M. White, Smoothed particle hydrodynamics for galaxyformation simulations: improved treatments of multiphase gas, of star formation and of supernovae feedback, MNRAS 345 (2003) 561-574.

[138] L. Tornatore, S. Borgani, V. Springel, F. Matteucci, N. Menci, G. Murante, Cooling and heating the intracluster medium in hydrodynamical simulations, MNRAS 342 (2003) 1025-1040.

[139] F. C. van den Bosch, T. Abel, L. Hernquist, The angular momentum of gas in protogalaxies - II. The impact of pre-heating, MNRAS 346 (2003) 177-185.

[140] P. C. Clark, I. A. Bonnell, Star formation in transient molecular clouds, MNRAS 347 (2004) L36-L40. 
[141] S. T. Kay, The entropy distribution in clusters: evidence of feedback?, MNRAS 347 (2004) L13-L17.

[142] T. J. Cox, J. Primack, P. Jonsson, R. S. Somerville, Generating Hot Gas in Simulations of Disk-Galaxy Major Mergers, ApJL 607 (2004) L87-L90.

[143] J. E. Dale, I. A. Bonnell, C. J. Clarke, M. R. Bate, Photoionizing feedback in star cluster formation, MNRAS 358 (2005) 291-304.

[144] M. Hoeft, G. Yepes, S. Gottlöber, V. Springel, Dwarf galaxies in voids: suppressing star formation with photoheating, MNRAS 371 (2006) 401-414.

[145] A. Cattaneo, J. Blaizot, D. H. Weinberg, D. Kereš, S. Colombi, R. Davé, J. Devriendt, B. Guiderdoni, N. Katz, Accretion, feedback and galaxy bimodality: a comparison of the GalICS semi-analytic model and cosmological SPH simulations, MNRAS 377 (2007) 63-76.

[146] V. Springel, L. Hernquist, Cosmological smoothed particle hydrodynamics simulations: the entropy equation, MNRAS 333 (2002) 649-664.

[147] J. J. Monaghan, SPH compressible turbulence, MNRAS 335 (2002) 843-852.

[148] J. J. Monaghan, On the problem of penetration in particle methods, Journal of Computational Physics 82 (1989) 1-15.

[149] M. Steinmetz, GRAPESPH: cosmological smoothed particle hydrodynamics simulations with the special-purpose hardware GRAPE, MNRAS 278 (1996) $1005-1017$.

[150] R. S. Klessen, A. Burkert, The Formation of Stellar Clusters: Gaussian Cloud Conditions. I., ApJS 128 (2000) 287-319.

[151] M. Wetzstein, A. F. Nelson, T. Naab, A. Burkert, VINE - A numerical code for simulating astrophysical systems using particles I: Description of the physics and the numerical methods, ArXiv e-prints.

[152] P. A. Thomas, H. M. P. Couchman, Simulating the formation of a cluster of galaxies, MNRAS 257 (1992) 11-31.

[153] S. Kunze, R. Speith, H. Riffert, Reproducing superhumps and gamma-shifts of SU UMa stars with SPH simulations, MNRAS 289 (1997) 889-897.

[154] D. J. Price, Modelling discontinuities and Kelvin-Helmholtz instabilities in SPH, Journal of Computational Physics 227 (2008) 10040-10057.

[155] P. Español, M. Revenga, Smoothed dissipative particle dynamics, Phys. Rev. E 67 (2) (2003) 026705.

[156] M. S. Fulbright, W. Benz, M. B. Davies, A method of smoothed particle hydrodynamics using spheroidal kernels, ApJ 440 (1995) 254-262.

[157] P. R. Shapiro, H. Martel, J. V. Villumsen, J. M. Owen, Adaptive Smoothed Particle Hydrodynamics, with Application to Cosmology: Methodology, ApJS 103 (1996) 269. 
[158] J. M. Owen, J. V. Villumsen, P. R. Shapiro, H. Martel, Adaptive Smoothed Particle Hydrodynamics: Methodology. II., ApJS 116 (1998) 155.

[159] J. J. Monaghan, Extrapolating B. Splines for Interpolation, Journal of Computational Physics 60 (1985) 253.

[160] D. Fulk, D. Quinn, An Analysis of 1-D Smoothed Particle Hydrodynamics Kernels, Journal of Computational Physics 126 (1996) 165-180.

[161] R. M. Cabezon, D. Garcia-Senz, A. Relano, A one-parameter family of interpolating kernels for smoothed particle hydrodynamics studies, Journal of Computational Physics 227 (2008) 8523-8540.

[162] J. I. Read, T. Hayfield, O. Agertz, Resolving mixing in Smoothed Particle Hydrodynamics, ArXiv e-prints.

[163] B. W. Ritchie, P. A. Thomas, Multiphase smoothed-particle hydrodynamics, MNRAS 323 (2001) 743-756.

[164] R. A. Gingold, J. J. Monaghan, Binary fission in damped rotating polytropes, MNRAS 184 (1978) 481-499.

[165] R. A. Gingold, J. J. Monaghan, Kernel estimates as a basis for general particle methods in hydrodynamics, Journal of Computational Physics 46 (1982) 429453.

[166] M. Steinmetz, E. Mueller, On the capabilities and limits of smoothed particle hydrodynamics, A \&A 268 (1993) 391-410.

[167] L. D. Landau, E. M. Lifshitz, Fluid mechanics, Course of theoretical physics, Oxford: Pergamon Press, 1959, 1959.

[168] G. Whitham, Linear and non-linear waves, John Wiley, New York, 1974.

[169] F. Shu, Gas Dynamics, University Science Books, 1992.

[170] W. H. Press, B. P. Flannery, S. A. Teukolsky, W. T. Vetterling, Numerical Recipes, Cambridge University Press, New York, 1992.

[171] J. VonNeumann, R. D. Richtmyer, A Method for the Numerical Calculation of Hydrodynamic Shocks, Journal of Applied Physics 21 (1950) 232-237.

[172] S.-I. Inutsuka, Reformulation of Smoothed Particle Hydrodynamics with Riemann Solver, Journal of Computational Physics 179 (2002) 238-267.

[173] S.-H. Cha, A. P. Whitworth, Implementations and tests of Godunov-type particle hydrodynamics, MNRAS 340 (2003) 73-90.

[174] S. M. Caramana, E., P. Whalen, Formulations of Artificial Viscosity for Multidimensional Shock Wave Computations, Journal of Computational Physics 144 (1998) 70-97.

[175] J. M. Owen, A tensor artificial viscosity for SPH, Journal of Computational Physics 201 (2004) 601-629. 
[176] R. Landshoff, A numerical method for treating fluid flow in the presence of shocks, Los Alamos National Laboratory Report, LA-1930.

[177] J. Monaghan, R. Gingold, J. Comp. Phys. 52 (1983) 374.

[178] G. Sod, A survey of several finite difference methods for systems of nonlinear hyperbolic conservation laws, J. Comput. Phys. 43 (1978) 1-31.

[179] D. S. Balsara, Asymmetries in extragalactic radio sources, Ph.D. thesis, AA(Illinois Univ., Urbana-Champaign.) (1991).

[180] J. F. Navarro, M. Steinmetz, The Effects of a Photoionizing Ultraviolet Background on the Formation of Disk Galaxies, ApJ 478 (1997) 13.

[181] S. Rosswog, M. B. Davies, F.-K. Thielemann, T. Piran, Merging neutron stars: asymmetric systems, A\&A 360 (2000) 171-184.

[182] K. Dolag, F. Vazza, G. Brunetti, G. Tormen, Turbulent gas motions in galaxy cluster simulations: the role of smoothed particle hydrodynamics viscosity, MNRAS 364 (2005) 753-772.

[183] J. J. Monaghan, SPH and Riemann Solvers, Journal of Computational Physics 136 (1997) 298-307.

[184] W. F. Noh, Errors for calculations of strong shocks using an artificial viscosity and an artificial heat flux, Journal of Computational Physics 72 (1987) 78-120.

[185] O. Agertz, B. Moore, J. Stadel, D. Potter, F. Miniati, J. Read, L. Mayer, A. Gawryszczak, A. Kravtsov, A. Nordlund, F. Pearce, V. Quilis, D. Rudd, V. Springel, J. Stone, E. Tasker, R. Teyssier, J. Wadsley, R. Walder, Fundamental differences between SPH and grid methods, MNRAS 380 (2007) 963-978.

[186] R. L. Burden, J. D. Faires, Numerical analysis, 7th ed., Pacific Grove, CA: Brooks/Cole, 2001. ISBN 0534382169, 2001.

[187] D. H. Porter, A study of hierarchical clustering of galaxies in an expanding universe, Ph.D. thesis, AA(California Univ., Berkeley.) (1985).

[188] M. W. J. Ewell, Dissipative N-body simulations of the formation of single galaxies in a cold dark matter cosmology, Ph.D. thesis, AA(Princeton Univ., NJ.) (1988).

[189] R. S. Klessen, F. Heitsch, M.-M. Mac Low, Gravitational Collapse in Turbulent Molecular Clouds. I. Gasdynamical Turbulence, ApJ 535 (2000) 887-906.

[190] S. Rosswog, Mergers of Neutron Star-Black Hole Binaries with Small Mass Ratios: Nucleosynthesis, Gamma-Ray Bursts, and Electromagnetic Transients, ApJ 634 (2005) 1202-1213.

[191] L. Verlet, Computer experiments on classical fluids. I Thermodynamical properties of Lennard-Jones molecules, Phys. Rev 159 (1967) 98-103. 
[192] B. Leimkuhler, S. Reich, Simulating Hamiltonian Dynamics, 1st Edition, Cambridge University Press, Cambridge, 2004.

[193] E. Hairer, C. Lubich, G. Wanner, Geometric Numerical Integration, 2nd Edition, Springer, Berlin, 2006.

[194] L. Landau, E. Lifshitz, Mechanik, 12th Edition, Akademieverlag, Berlin, 1976.

[195] H. Goldstein, C. Poole, J. Safko, Classical mechanics, Classical mechanics (3rd ed.) by H. Goldstein, C. Poolo, and J. Safko. San Francisco: Addison-Wesley, 2002., 2002.

[196] E. Fehlberg, New High-Order Runge-Kutta Formulas with Step Size Control for Systems of First- and Second-Order Differential Equations 44.

[197] E. Fehlberg, New one-step integration methods of high order accuracy applied to some problems in celestial mechanics, Tech. rep., NASA technical report TR R-248 (1966).

[198] E. Fehlberg, Low-order classical Runge-Kutta formulas with step size control and their application to some heat transfer problems, Tech. rep., NASA technical report TR R-315 (1969).

[199] M. Griebel, S. Knapek, G. Zumbusch, Numerical simulation in molecular dynamics, 1st Edition, Springer, Berlin, 2007.

[200] T. R. Saitoh, J. Makino, A Necessary Condition for Individual Time Steps in SPH Simulations, ApJL 697 (2009) L99-L102.

[201] J. J. Monaghan, D. J. Price, Variational principles for relativistic smoothed particle hydrodynamics, MNRAS 328 (2001) 381-392.

[202] C. Eckart, Variation principles of hydrodynamics, Physics of Fluids 3 (1960) 421.

[203] D. Price, J. Monaghan, An energy-conserving formalism for adaptive gravitational force softening in sph and n-body codes, MNRAS 374 (2007) 1347.

[204] R. Nelson, J. Papaloizou, Variable smoothing lengths and energy conservation in smooth particle hydrodynamics, MNRAS 270 (1994) 1.

[205] P. Mann, A relativistic smoothed particle hydrodynamics method tested with the shock tube, Computer Physics Communications.

[206] P. Mann, Smoothed particle hydrodynamics applied to relativistic spherical collapse, Journal of Computational Physics 107 (1993) 188-198.

[207] P. Laguna, W. A. Miller, W. H. Zurek, Smoothed particle hydrodynamics near a black hole, ApJ 404 (1993) 678-685.

[208] E. Rantsiou, S. Kobayashi, P. Laguna, F. Rasio, Mergers of Black Hole Neutron Star binaries. I. Methods and First Results, ArXiv Astrophysics eprints. 
[209] A. Kheyfets, W. A. Miller, W. H. Zurek, Covariant smoothed particle hydrodynamics on a curved background, Physical Review D 41 (1990) 451-454.

[210] J. E. Chow, J. Monaghan, Ultrarelativistic sph, J. Computat. Phys. 134 (1997) 296.

[211] S. Siegler, H. Riffert, Smoothed Particle Hydrodynamics Simulations of Ultrarelativistic Shocks with Artificial Viscosity, ApJ 531 (2000) 1053-1066.

[212] S. Siegler, Entwicklung und untersuchung eines smoothed particle hydrodynamics verfahrens für relativistische strömungen, Ph.D. thesis, Eberhard-Karls-Universität Tübingen (2000).

[213] S. Ayal, T. Piran, R. Oechslin, M. B. Davies, S. Rosswog, Post-Newtonian Smoothed Particle Hydrodynamics, ApJ 550 (2001) 846-859.

[214] J. A. Faber, F. A. Rasio, Post-Newtonian SPH calculations of binary neutron star coalescence: Method and first results, Phys. Rev. D 62 (6) (2000) 064012.

[215] J. A. Faber, F. A. Rasio, J. B. Manor, Post-Newtonian smoothed particle hydrodynamics calculations of binary neutron star coalescence. II. Binary mass ratio, equation of state, and spin dependence, Phys. Rev. D 63 (4) (2001) 044012.

[216] J. A. Faber, F. A. Rasio, Post-Newtonian SPH calculations of binary neutron star coalescence. III. Irrotational systems and gravitational wave spectra, Phys. Rev. D 65 (8) (2002) 084042.

[217] J. Isenberg, Waveless approximation theories of gravity, unpublished.

[218] J. R. Wilson, G. Mathews, P. Marronetti, Phys. Rev. D54 (1996) 1317.

[219] R. Oechslin, S. Rosswog, F.-K. Thielemann, Conformally flat smoothed particle hydrodynamics application to neutron star mergers, Phys. Rev. D 65 (10) (2002) 103005.

[220] J. A. Faber, P. Grandclément, F. A. Rasio, Mergers of irrotational neutron star binaries in conformally flat gravity, Phys. Rev. D 69 (12) (2004) 124036.

[221] J. A. Faber, T. W. Baumgarte, S. L. Shapiro, K. Taniguchi, F. A. Rasio, Dynamical evolution of black hole-neutron star binaries in general relativity: Simulations of tidal disruption, Phys. Rev. D 73 (2) (2006) 024012.

[222] V. Fock, Theory of Space, Time and Gravitation, Pergamon, Oxford, 1964.

[223] M. L. Norman, K.-H. Winkler, Why ultrarelativistic numerical hydrodynamics is difficult, in: K.-H. Winkler, M. L. Norman (Eds.), Astrophysical Radiation Hydrodynamics, Reidel, Berlin, 1986.

[224] J. Marti, E. Müller, J. Comp. Phys. 123 (1996) 1.

[225] L. Del Zanna, N. Bucciantini, An efficient shock-capturing central-type scheme for multidimensional relativistic flows. I. Hydrodynamics, A\&A 390 (2002) 1177-1186. 
[226] J. M. Marti, E. Müller, Numerical Hydrodynamics in Special Relativity, Living Reviews in Relativity 6 (2003) 7.

[227] S. Rosswog, Conservative, special-relativistic smooth particle hydrodynamics, ArXiv e-prints.

[228] B. Schutz, A first course in general relativity, 1st Edition, Cambridge University Press, Cambridge, 1989. 